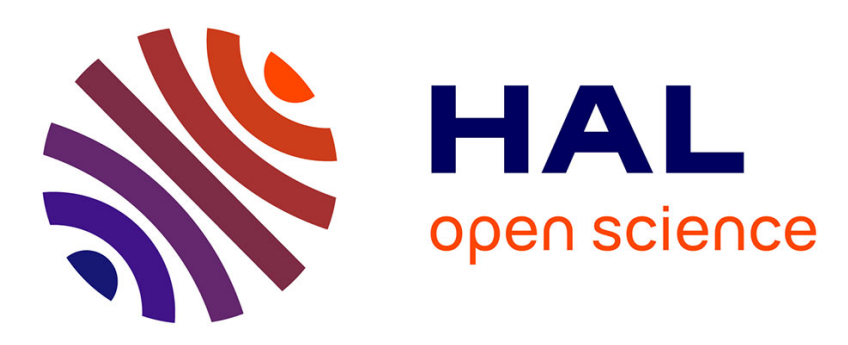

\title{
Transfer of conservative discrete differential operators between staggered grids: construction and duality relations
}

\author{
Stella Krell, Sebastian Minjeaud, Julie Llobell, Thierry Goudon
}

\section{To cite this version:}

Stella Krell, Sebastian Minjeaud, Julie Llobell, Thierry Goudon. Transfer of conservative discrete differential operators between staggered grids: construction and duality relations. IMA Journal of Numerical Analysis, 2022, 42 (3), pp.2459-2504. 10.1093/imanum/drab042 . hal-03428267

\section{HAL Id: hal-03428267 https://hal.science/hal-03428267}

Submitted on 15 Nov 2021

HAL is a multi-disciplinary open access archive for the deposit and dissemination of scientific research documents, whether they are published or not. The documents may come from teaching and research institutions in France or abroad, or from public or private research centers.
L'archive ouverte pluridisciplinaire HAL, est destinée au dépôt et à la diffusion de documents scientifiques de niveau recherche, publiés ou non, émanant des établissements d'enseignement et de recherche français ou étrangers, des laboratoires publics ou privés. 


\title{
Transfer of conservative discrete differential operators between staggered grids: construction and duality relations
}

\author{
Thierry Goudon*, Stella Krell † Julie Llobell \\ Université Côte d'Azur, Inria, CNRS, LJAD \\ Parc Valrose, F-06108 Nice, France
}

\begin{abstract}
This paper is concerned with the construction of a discrete conservative operator on a mesh, knowing a conservative operator on a different mesh. We analyse the possibility of defining such a transfer based on the preservation of conservation properties, and provide practical procedures, which can be effectively implemented for schemes that work on staggered grids. We show that such a transfer can be applied to obtain a discrete balance equation on a mesh starting from a discrete balance equation on a different mesh and to prove local discrete duality relations for standard discrete operators defined on staggered grids.
\end{abstract}

Keywords: Staggered grids. Finite Volume method. Discrete Duality Finite Volume. Divergence problem.

\section{Contents}

1 Introduction $\quad 2$

2 Cell-average of divergence operators 4

2.1 Existence of the average operator . . . . . . . . . . . . . . . 4

2.2 Practical construction of the average operator . . . . . . . . . . . 10

2.2.1 Construction of the function $\omega_{K} \ldots \ldots \ldots 11$

2.2.2 Expression of the transfer fluxes for quadrangular cells . . . . . . 17

*thierry.goudon@inria.fr

†stella.krell@unice.fr

$\ddagger$ julie.llobell@unice.fr

$\S$ sebastian.minjeaud@unice.fr 
3 Examples of applications $\quad 19$

3.1 A simple but illuminating example . . . . . . . . . . . . . . . . . 19

3.2 Transfer of conservative equations . . . . . . . . . . . . . 22

3.3 Local discrete duality relations . . . . . . . . . . . . . . . . . . 24

3.3.1 Framework and application of Theorem $2.7 \ldots \ldots . \ldots 24$

3.3 .2 Discrete operators . . . . . . . . . . . . . . . . . . 29

3.3.3 Local discrete duality relations . . . . . . . . . . . . . . . 34

A Proof of Lemma 2.2 with $H^{1}$ regularity $\quad 39$

B Proof of Lemma 2.9 40

C A few basic facts on $\mathbb{P}^{1}$ basis functions $\quad 42$

\section{Introduction}

This work is motivated by a general question which arises in the design and analysis of numerical schemes that work on staggered grids. We focus on the two-dimensional framework: the computational domain $\Omega$ is covered by several tessellations, say

$$
\Omega=\bigcup_{K \in \mathfrak{T}} K \quad \text { and } \quad \Omega=\bigcup_{D \in \mathfrak{D}} D
$$

We wish to numerically compute several quantities which are naturally stored on the different grids $\mathfrak{T}$ and $\mathfrak{D}$. We specifically have in mind Finite Volume approaches where the numerical unknowns are constant over the cells. For instance, we are interested in the velocity $u_{D}$ and the density $\rho_{K}$ of a fluid, governed by the (barotropic) Euler or Navier-Stokes equations. The method updates the quantities through a suitable definition of numerical fluxes, which mimics the exchanges (of mass, of momentum...) at the boundaries of the cells. Hence, in the example of Euler or Navier-Stokes equations, we have to design momentum fluxes $G_{D, \mathfrak{s}}$ and mass fluxes $F_{K, \sigma}$ on the interfaces ( $\mathfrak{s}$ and $\sigma$ ) of the cells of both grids. The construction and the analysis of the scheme require to transfer the balance equations from one mesh to the other one. This is specifically the case when we aim at establishing a local energy balance since the total energy involves quantities that are discretized on both meshes [19, 23]. We propose in this article a general solution to define a divergence operator on a mesh based on a suitable average of a divergence operator known on another mesh.

Assume that we have at hand fluxes $F_{K, \sigma}$ defined on the edges $\sigma$ of the cells $K$ that belong to the mesh $\mathfrak{T}$. We denote by $\operatorname{div}^{K}$ the following operator

$$
\operatorname{div}^{K}=\frac{1}{|K|} \sum_{\sigma \in \partial K} F_{K, \sigma} .
$$

From $F_{K, \sigma}$, we aim at defining fluxes $F_{D, \mathfrak{s}}$ on the edges $\mathfrak{s}$ of the cells $D$ of the mesh $\mathfrak{D}$ such that the operator

$$
\operatorname{div}^{D}=\frac{1}{|D|} \sum_{\mathfrak{s} \in \partial D} F_{D, \mathfrak{s}}
$$


can be expressed as the average on the cell $D$ of the operators $\operatorname{div}^{K}$ for the cells $K \in \mathfrak{T}$ such that $K \cap D \neq \emptyset$. This construction is governed by the following concerns:

(Q1) Can we define consistently a transfer of information between the grids ? The consistency here refers to the preservation of certain fundamental properties on both grids. To be more specific, we consider a quantity $\rho$ (the mass density, say) discretized on the mesh $\mathfrak{T}$ : it is updated on each cell $K \in \mathfrak{T}$ according to a conservation equation

$$
\frac{\bar{\rho}_{K}-\rho_{K}}{\Delta t}+\operatorname{div}^{K}=0,
$$

where $\Delta t$ stands for the time step, with given conservative numerical fluxes $F_{K, \sigma}$. Namely, if $\sigma=K \mid K^{\prime}$ is the interface between two cells $K$ and $K^{\prime}$, we have

$$
F_{K, \sigma}=-F_{K^{\prime}, \sigma} .
$$

We now define an averaged quantity on the twin grid $\mathfrak{D}$ as follows

$$
\rho_{D}=\sum_{\substack{K \in \mathfrak{T} \\ K \cap D \neq \emptyset}} \frac{|K \cap D|}{|D|} \rho_{K} .
$$

We wish to define new numerical mass fluxes $F_{D, \mathfrak{s}}$ on $D$ such that a discrete mass conservation

$$
\bar{\rho}_{D}-\rho_{D}=-\Delta t \sum_{\mathfrak{s} \in \partial D} F_{D, \mathfrak{s}},
$$

still holds, with $F_{D, \mathfrak{s}}$ satisfying the conservation property

$$
F_{D, \mathfrak{s}}=-F_{D^{\prime}, \mathfrak{s}}
$$

for any interface $\mathfrak{s}=D \mid D^{\prime}$.

(Q2) Can we derive discrete duality relations that mimic the equalities

$$
\int_{\mathscr{O}} u \cdot \nabla \phi \mathrm{d} x+\int_{\mathscr{O}} \operatorname{div} u \phi \mathrm{d} x=\int_{\mathscr{O}} \operatorname{div}(u \phi) \mathrm{d} x=\int_{\partial \mathscr{O}} u \phi \cdot \nu \mathrm{d} \sigma
$$

at the discrete level (with $\mathscr{O}$ an open set of $\Omega$ ) ? We expect to recover such duality relations on the grids $\mathfrak{T}$ and $\mathfrak{D}$, involving the discrete operators $\operatorname{div}^{K}$ and $\operatorname{div}^{D}$, and numerical quantities $u, \phi$ that live on the different grids.

Question (Q1) is addressed in [3] and solutions are proposed in the framework of Crouzeix-Raviart and Rannacher-Turek discretizations. We also refer the reader to [5] which deals with quadrilateral cells, and to [17] where the problem is addressed in the framework of Discrete Duality Finite Volume (DDFV) methods for non-homogeneous Navier-Stokes equations. Question (Q2) appears when discretizing the Euler system on staggered grids: the full energy mixes up quantities stored on different grids, and checking the consistency of the scheme with the total energy conservation relies on such duality relations $[19,23]$.

In Section 2, we consider the construction of the fluxes $F_{D, \mathfrak{s}}$ from the $F_{K, \sigma}$ 's, dealing with fully general meshes $\mathfrak{T}$ and $\mathfrak{D}$. We thus generalize the solutions proposed 
in $[3,5,17]$. In fact, our argument is two-fold. On the one hand the construction relies on the capability of solving a boundary-value problem for the divergence operator, and properties of the solution can be deduced from general PDEs statements coming from [4]. On the other hand, we also propose approaches to compute effectively the new fluxes, which is important in the perspective of designing schemes on staggered grids. Complementary to the general strategy, these simple and explicit constructions provide practical tools, possibly applicable for particular subclasses of meshes.

In Section 3.2 we go back to the conservation issue (Q1) and the local discrete duality relation (Q2) is addressed in Section 3.3. The latter requires some geometric relations in the construction of the two meshes $\mathfrak{T}$ and $\mathfrak{D}$, which in particular apply to the Two-Point Flux Approximation (TPFA) and the DDFV discretizations.

\section{Cell-average of divergence operators}

We prove in this section the main result of the article. It is presented in Section 2.1 and it states the existence of the fluxes $F_{D, \mathfrak{s}}$ mentionned in the introduction. The proof is based on regularity results for the inverse of the (continuous) divergence operator and does not offer a practical construction of the fluxes. Such a construction becomes important if these fluxes are not only used for the purpose of numerical analysis but also they are incorporated in the definition of the scheme which effectively runs to approximate the solutions of the equations. Several constructions are presented in Section 2.2. The discussion makes repeated use of the following elementary claim.

Lemma 2.1. Let $K$ be a polygon in $\mathbb{R}^{2}$, with edges $\sigma_{1}, \ldots, \sigma_{n}$. We denote by $\mathbf{n}_{j}$ the unit outward normal on the edge $\sigma_{j}$. Then, we have

$$
\sum_{j=1}^{n}\left|\sigma_{j}\right| \mathbf{n}_{j}=0
$$

Proof. We denote $\mathbf{n}_{j}=\left(\mathbf{n}_{j, 1}, \mathbf{n}_{j, 2}\right)$. Let $\mathbf{e}_{1}=(1,0), \mathbf{e}_{2}=(0,1)$. Denoting similarly the constant function over $K$, the Green formula yields

$$
\int_{K} \boldsymbol{\nabla} \cdot\left(\mathbf{e}_{i}\right)=0=\sum_{j=1}^{n} \int_{\sigma_{j}} \mathbf{e}_{i} \cdot \mathbf{n}_{j}=\sum_{j=1}^{n}\left|\sigma_{j}\right| \mathbf{n}_{j, i},
$$

which is the componentwise expression of the statement.

\subsection{Existence of the average operator}

The proof of the existence of the average operator relies on the following lemma. This statement is reminiscent of ideas presented in [3] where the case of triangular or rectangular cells is treated. Here and in Section 2.2, we consider general polygonal domains. We shall work with the space $H_{\text {div }}(\mathscr{O})$, which contains the vector fields $\mathbf{v}$ defined on an open set $\mathscr{O}$ and such that $\mathbf{v} \in L^{2}(\mathscr{O}), \nabla \cdot \mathbf{v} \in L^{2}(\mathscr{O})$. In what follows we shall work with closed subsets $X$ in $\mathbb{R}^{2}$ (typically cells of different meshes) and $\stackrel{\circ}{X}$ stands for the topological interior of $X$. 
Lemma 2.2. Let us consider a (bounded, simply-connected) star-shaped polygon $K$ in $\mathbb{R}^{2}$. We denote by $\mathscr{E}^{K}$ the set of the edges $\sigma$ of $K$. For all edge $\sigma \in \mathscr{E}^{K}$, we denote by $\mathbf{n}_{K, \sigma}$ the unit normal vector to $\sigma$ pointing outwards the cell $K$. At each $\sigma \in \mathscr{E}^{K}$ it is associated a given scalar (flux-type) quantity $\mathcal{X}_{K, \sigma}$. Then, there exists a function $\boldsymbol{\omega}_{K} \in H_{\operatorname{div}}(\stackrel{\circ}{K})$ such that

$$
\boldsymbol{\nabla} \cdot \boldsymbol{\omega}_{K}=\frac{1}{|K|} \sum_{\sigma \in \mathscr{E} K} \mathcal{X}_{K, \sigma} \quad \text { a.e. on } K
$$

and

$$
\int_{\sigma} \boldsymbol{\omega}_{K} \cdot \mathbf{n}_{K, \sigma}=\mathcal{X}_{K, \sigma}, \quad \forall \sigma \in \mathscr{E}^{K}
$$

Proof. A natural approach consists in looking for $\boldsymbol{\omega}_{K}$ as the gradient of a scalar potential function $\Phi$, which is thus defined as the solution of a Poisson problem with suitable Neumann boundary conditions. This approach provides, by the standard variational argument, a solution $\Phi \in H^{1}(\stackrel{\circ}{K})$ and thus $\boldsymbol{\omega}_{K}=\nabla \Phi \in H_{\text {div }}(\stackrel{\circ}{K})$.

In the specific case of a convex domain $K$, the regularity is strengthened: the potential $\Phi$ lies in $H^{2}(\stackrel{\circ}{K})$, and thus $\omega_{K}$ has the full $H^{1}$ regularity. This argument fails when the domain $K$ is not convex, see [20, Theorem 2.3.7 (case ii), Theorem 2.4.3 and Remark 2.4.5].

It is worth noting that solutions of (2)-(3) are not uniquely defined in general (for instance on the unit square with the four fluxes $\mathcal{X}_{K, \sigma}=0, \boldsymbol{\omega}_{K}=(0,0)$ and $\boldsymbol{\omega}_{K}=\left(x_{1}\left(1-x_{1}\right)\left(1-2 x_{2}\right), x_{2}\left(1-x_{2}\right)\left(1-2 x_{1}\right)\right)$ are both solutions.

Remark 2.3. The $H_{\text {div }}$ regularity for $\boldsymbol{\omega}_{K}$ as stated in Lemma 2.2 turns out to be enough for our purposes. However, it is possible to find a solution $\boldsymbol{\omega}_{K}$ with the $H^{1}$ regularity. Indeed, let us consider the solution $\mathbf{u}$ of the following boundary value problem for the divergence operator

$$
\left\{\begin{aligned}
\boldsymbol{\nabla} \cdot \mathbf{u} & =f, & & \text { in } \stackrel{\circ}{K}, \\
\mathbf{u} & =\mathbf{g}, & & \text { on } \partial K .
\end{aligned}\right.
$$

This problem admits solutions under suitable regularity and compatibility assumptions on the functions $f$ and $\mathbf{g}$ [4, Theorem 7.1]. Suitable functions $f$ and $\mathbf{g}$ which will lead to the desired conclusion are given in Appendix A. In some cases, practical constructions of the function $\boldsymbol{\omega}_{K}$ provided in Section 2.2.1 also lead to full $H^{1}$ regularity.

Remark 2.4. The proof does not use any condition on the orientation of one edge compared to the others. Thus the result still applies for degenerate polygonal domains with several (consecutive) aligned vertices.

Remark 2.5. The construction of a function with the $H_{\text {div }}$ or $H^{1}$ regularity defined by given fluxes on the boundary of a domain naturally appears for defining efficient a posteriori estimators. Indeed, a finite volume scheme constructs a piecewise constant function $p^{h}$ intended to approximate the solution of an elliptic equation $\nabla \cdot(A \nabla p)=f \in$ 
$L^{2}$; this quantity does not satisfy the natural regularity of the solution of the problem: in general it does not belong to $H^{1}$ and $A \nabla p^{h} \notin H_{\text {div }}$ neither. From the discrete unknowns $p^{h}$, one needs to define a more regular function which can be used to evaluate the error [16]. Except on particular mesh geometries, the practical construction of such functions, and its robustness, is a delicate issue. We refer the reader to [13, 14, 26, 27] for the development of a unified viewpoint on these questions. Despite a natural connection, it is not direct to adapt these results to the present purpose. Here, we both use the general statement Lemma 2.2 to derive useful formula for the transfer of information between different grids, and we propose practical constructions, which have been effectively implemented for solving PDE systems of fluid mechanics [17, 18, 19, 23]

With Lemma 2.2 at hand, we now consider, as mentioned in the introduction, two meshes $\mathfrak{T}$ and $\mathfrak{D}$ of the $2 \mathrm{D}$ computational domain $\Omega$ :

$$
\Omega=\bigcup_{K \in \mathfrak{T}} K \quad \text { and } \quad \Omega=\bigcup_{D \in \mathfrak{D}} D
$$

For the time being, we do not assume that the construction of $\mathfrak{D}$ depends on the geometry of $\mathfrak{T}$. We only assume that the interior edges of $\mathfrak{T}$ and $\mathfrak{D}$ do not overlap (see Assumption 2.6). For an element $K \in \mathfrak{T}$ (resp. $D \in \mathfrak{D}$ ), we denote by $\mathscr{E}^{K}$ (resp. $\mathscr{E}^{D}$ ) the set of the edges $\sigma$ of $K$ (resp. the edges $\mathfrak{s}$ of $D$ ). We denote by $\mathscr{E}^{\mathfrak{T}}$ and $\mathscr{E}^{\mathfrak{D}}$ the set of all edges of the elements in $\mathfrak{T}$ and in $\mathfrak{D}$ respectively:

$$
\mathscr{E}^{\mathfrak{T}}=\bigcup_{K \in \mathfrak{T}} \mathscr{E}^{K} \quad \text { and } \quad \mathscr{E}^{\mathfrak{D}}=\bigcup_{D \in \mathfrak{D}} \mathscr{E}^{D}
$$

The edges on the boundary of the domain $\Omega$ are gathered in $\mathscr{E}^{\mathfrak{T} \text {,ext }}$ and $\mathscr{E}^{\mathcal{D}}$,ext

$$
\mathscr{E}^{\mathfrak{T}, \text { ext }}=\left\{\sigma \in \mathscr{E}^{\mathfrak{T}} ; \sigma \subset \partial \Omega\right\} \quad \text { and } \quad \mathscr{E}^{\mathfrak{D}, \text { ext }}=\left\{\mathfrak{s} \in \mathscr{E}^{\mathfrak{D}} ; \mathfrak{s} \subset \partial \Omega\right\}
$$

while $\mathscr{E}^{\mathfrak{T} \text {,int }}=\mathscr{E}^{\mathfrak{T}} \backslash \mathscr{E}^{\mathfrak{T} \text {,ext }}$ and $\mathscr{E}^{\mathfrak{D} \text {,int }}=\mathscr{E}^{\mathfrak{D}} \backslash \mathscr{E}^{\mathfrak{D} \text {,ext }}$ stand for the sets of interior edges.

The mesh $\mathfrak{D}$ splits the edges of a cell $K \in \mathfrak{T}$ in several sub-edges (see Fig. 1). We will consider that it gives rise to a "degenerate" polygonal cell $K^{\mathfrak{D}}$ which coincides with $K$, "degenerate" meaning here that several vertices may be aligned. Hence, we define the following set of edges

$$
\mathfrak{E}_{\mathfrak{D}}^{K}=\bigcup_{\sigma \in \mathscr{E}^{K}} \mathfrak{E}_{\mathfrak{D}}^{\sigma} \text { with } \mathfrak{E}_{\mathfrak{D}}^{\sigma}=\left\{\sigma \cap D, D \in \mathfrak{D}^{\sigma}\right\},
$$

where $\mathfrak{D}^{\sigma}$ is the set of the cells $D \in \mathfrak{D}$ intersecting the edge $\sigma$

$$
\mathfrak{D}^{\sigma}=\{D \in \mathfrak{D} ; \sigma \cap D \neq \emptyset\} .
$$

Thus, the boundary of $K \in \mathfrak{T}$ can be described as the boundary of $K^{\mathfrak{D}}$ (since the two cells coincide)

$$
\partial K=\bigcup_{\sigma \in \mathscr{E}^{K}} \sigma=\bigcup_{\sigma^{D} \in \mathfrak{E}_{\mathfrak{Q}}^{K}} \sigma^{D}=\partial K^{\mathfrak{D}}
$$




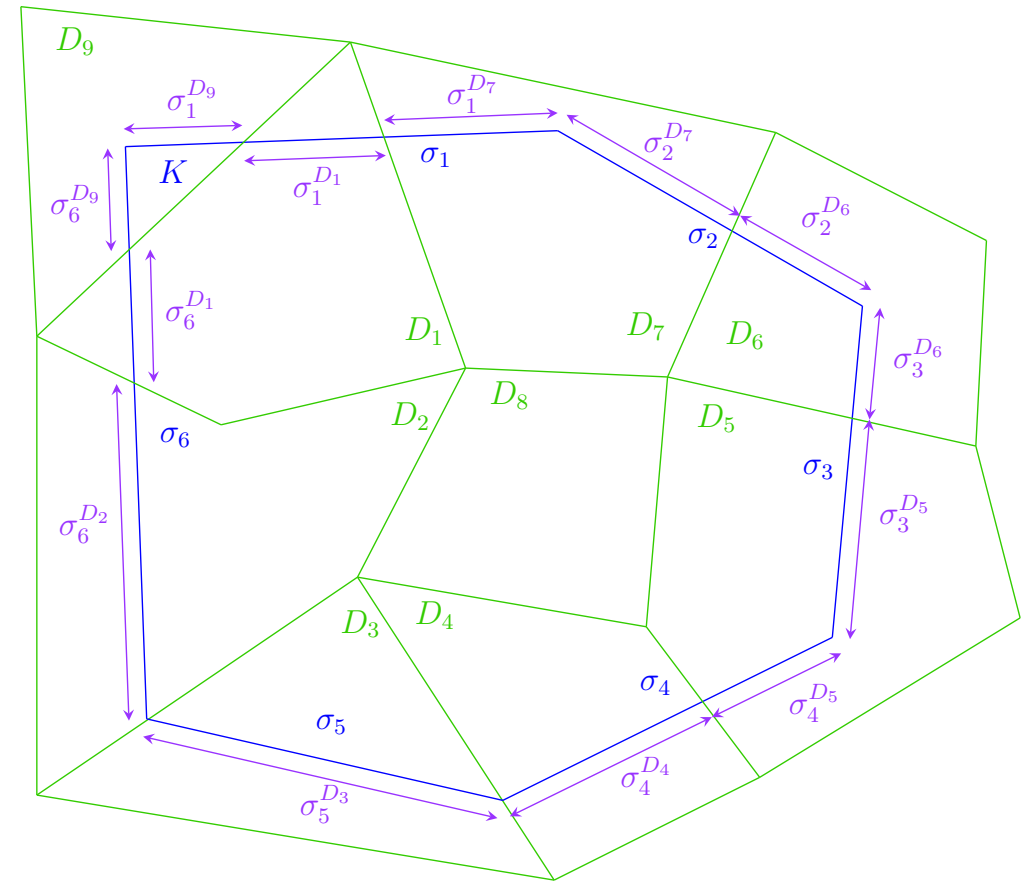

Figure 1: Sub-edges $\sigma^{D}$ (splitting of the edges $\sigma \in \mathscr{E}^{\mathfrak{T}}$ by the mesh $\mathfrak{D}$ ).

We define $\mathfrak{E}_{\mathfrak{D}}^{\mathfrak{T}}$ as the set of all sub-edges and we gather in $\mathfrak{E}_{\mathfrak{D}}^{\mathfrak{T}, \text { ext }}$ all the sub-edges on the boundary of the domain $\Omega$

$$
\mathfrak{E}_{\mathfrak{D}}^{\mathfrak{T}}=\bigcup_{\sigma \in \mathscr{E}^{\mathfrak{T}}} \mathfrak{E}_{\mathfrak{D}}^{\sigma}, \quad \mathfrak{E}_{\mathfrak{D}}^{\mathfrak{T}, \text { ext }}=\bigcup_{\sigma \in \mathscr{E}^{\mathfrak{T}, \text { ext }}} \mathfrak{E}_{\mathfrak{D}}^{\sigma}, \quad \mathfrak{E}_{\mathfrak{D}}^{\mathfrak{T}, \text { int }}=\mathfrak{E}_{\mathfrak{D}}^{\mathfrak{T}} \backslash \mathfrak{E}_{\mathfrak{D}}^{\mathfrak{T}, \text { ext }}
$$

Remark that we denote the elements of $\mathfrak{E}_{\mathfrak{D}}^{\mathfrak{T}}$ by $\sigma^{D}$ with a label $D \in \mathfrak{D}$ such that $\sigma^{D} \subset D$. This label is actually uniquely determined since in the sequel we adopt the following assumption which ensures that an element of $\mathfrak{E}_{\mathfrak{D}}^{\mathfrak{T}}$ can not be shared by several cells of $\mathfrak{D}$.

Assumption 2.6. We assume that the interior edges of $\mathfrak{T}$ and $\mathfrak{D}$ do not overlap

$$
\forall \sigma^{D} \in \mathfrak{E}_{\mathfrak{D}}^{\mathfrak{T}, \text { int }}, \forall \mathfrak{s} \in \mathscr{E}^{\mathfrak{D}}, \quad \sigma^{D} \not \subset \mathfrak{s} .
$$

The main result of the article can be stated as follows.

Theorem 2.7. Let $\mathfrak{T}$ and $\mathfrak{D}$ two meshes satisfy Assumption 2.6. Assume that (fluxtype) quantities $F_{K, \sigma^{D}} \in \mathbb{R}$ are given for all $K \in \mathfrak{T}$, for all $\sigma^{D} \in \mathfrak{E}_{\mathfrak{D}}^{K}$. For $K \in \mathfrak{T}$ and $\sigma \in \mathscr{E}^{K}$, we denote by $F_{K, \sigma}$ the sum

$$
F_{K, \sigma}=\sum_{\sigma^{D} \in \mathfrak{E}_{\mathfrak{O}}^{\sigma}} F_{K, \sigma^{D}}
$$


Then, there exist (flux-type) quantities $F_{D, \mathfrak{s}} \in \mathbb{R}$ for all $D \in \mathfrak{D}$, for all $\mathfrak{s} \in \mathscr{E}^{D}$ such that

$$
\begin{aligned}
& F_{D, \mathfrak{s}}=-F_{D^{\prime}, \mathfrak{s}} \text { if } \mathfrak{s}=D \mid D^{\prime} \in \mathscr{E}^{\mathfrak{D}, \text { int }} \text { is the edge between } D \text { and } D^{\prime} \text { (conservativity), } \\
& F_{D, \mathfrak{s}}=\sum_{K \in \mathfrak{T}} \sum_{\substack{\sigma^{D} \in \mathfrak{E}_{\mathfrak{\mathfrak { T }}, \text { ext }}^{\mathfrak{D}} \sigma^{D} \subset \mathfrak{F} \\
\sigma_{\mathfrak{D}}^{K}}} F_{K, \sigma^{D}}, \quad \text { if } \mathfrak{s} \in \mathscr{E}^{\mathfrak{D}, \text { ext }} \cap \mathscr{E}^{D},
\end{aligned}
$$

and

$$
|D| \operatorname{div}^{D}=\sum_{K \in \mathfrak{T}}|K \cap D| \operatorname{div}^{K}-\sum_{K \in \mathfrak{T}} \sum_{\substack{\sigma^{D} \in \mathfrak{E}_{\mathfrak{Q}}^{K} \\ \sigma^{D} \subset D, \sigma^{D} \not \supset \partial D}} F_{K, \sigma^{D}}, \quad \forall D \in \mathfrak{D},
$$

where

$$
\operatorname{div}^{D}=\frac{1}{|D|} \sum_{\mathfrak{s} \in \mathscr{E}^{D}} F_{D, \mathfrak{s}} \quad \text { and } \quad \operatorname{div}^{K}=\frac{1}{|K|} \sum_{\sigma \in \mathscr{E}^{K}} F_{K, \sigma}
$$

Proof. Let us consider a cell $K \in \mathfrak{T}$. Since the cells $K$ and $K^{\mathfrak{D}}$ coincide, we can apply Lemma 2.2 (see also Remark 2.4) to prove the existence of a function $\boldsymbol{\omega}_{K} \in H_{\text {div }}(\stackrel{\circ}{K})$ such that

$$
\boldsymbol{\nabla} \cdot \boldsymbol{\omega}_{K}=\frac{1}{|K|} \sum_{\sigma^{D} \in \mathfrak{E}_{\mathfrak{D}}^{K}} F_{K, \sigma^{D}}
$$

and

$$
\int_{\sigma^{D}} \boldsymbol{\omega}_{K} \cdot \mathbf{n}_{K, \sigma^{D}}=F_{K, \sigma^{D}}, \quad \forall \sigma^{D} \in \mathfrak{E}_{\mathfrak{D}}^{K} .
$$

Since the edges $\sigma^{D} \in \mathfrak{E}_{\mathfrak{D}}^{K}$ are defined by $\sigma^{D}=\sigma \cap D, \sigma \in \mathscr{E}^{K}, D \in \mathfrak{D}^{\sigma}$, we have

$$
\begin{aligned}
\sum_{\sigma^{D} \in \mathfrak{E}_{\mathfrak{O}}^{K}} F_{K, \sigma^{D}} & =\sum_{\sigma \in \mathscr{E}^{K}} \sum_{\sigma^{D} \in \mathfrak{E}_{\mathfrak{P}}^{\sigma}} F_{K, \sigma^{D}} \\
& =\sum_{\sigma \in \mathscr{E}^{K}} F_{K, \sigma} \\
& =|K| \operatorname{div}^{K} .
\end{aligned}
$$

Thus, the function $\boldsymbol{\omega}_{K}$ satisfies

$$
\boldsymbol{\nabla} \cdot \boldsymbol{\omega}_{K}=\operatorname{div}^{K}
$$

We can now set

$$
F_{D, \mathfrak{s}}=\sum_{K \in \mathfrak{T}} \int_{K \cap \mathfrak{s}} \boldsymbol{\omega}_{K} \cdot \mathbf{n}_{D, \mathfrak{s}} .
$$

These fluxes are well defined since they involve the normal trace of the $H_{\text {div }}$ function $\boldsymbol{\omega}_{K}$. The conservativity of these fluxes directly comes from the definition of the normal $\mathbf{n}_{D, \mathfrak{s}}$. Indeed, if $\mathfrak{s}=D \mid D^{\prime}$ is the edge between $D$ and $D^{\prime}$, we have $\mathbf{n}_{D, \mathfrak{s}}=-\mathbf{n}_{D^{\prime}, \mathfrak{s}}$. Moreover, if $\mathfrak{s} \in \mathscr{E}^{\mathfrak{D} \text {,ext }} \cap \mathscr{E}^{D}$ then

$$
\{K \cap \mathfrak{s} \neq \emptyset ; K \in \mathfrak{T}\}=\left\{\sigma^{D} \in \mathfrak{E}_{\mathfrak{D}}^{\mathfrak{T}, \text { ext }} ; \sigma^{D} \subset \mathfrak{s}\right\}
$$


and, if $\sigma^{D}=K \cap \mathfrak{s}$, the normal vectors $\mathbf{n}_{D, \mathfrak{s}}$ and $\mathbf{n}_{K, \sigma^{D}}$ both coincide with the outward

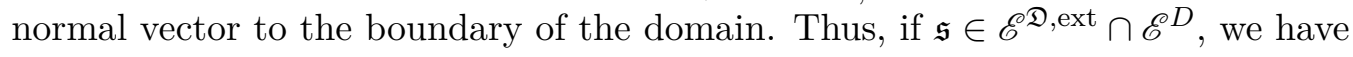

$$
\begin{aligned}
F_{D, \mathfrak{s}} & =\sum_{\substack{\sigma^{D} \in \mathfrak{E}_{\mathfrak{T}, \text { ext }} \\
\sigma^{D} \subset \mathfrak{s}}} \int_{\sigma^{D}} \boldsymbol{\omega}_{K} \cdot \mathbf{n}_{K, \sigma^{D}}, \\
& =\sum_{\substack{\sigma^{D} \in \mathfrak{F}_{\mathfrak{T}, \text { ext }} \\
\sigma^{D} \subset \mathfrak{s}}} F_{K, \sigma^{D}} .
\end{aligned}
$$

Let us now turn to the proof of (4). We consider a cell $D \in \mathfrak{D}$. For all $K \in \mathfrak{T}$, the Green formula applied on the polygonal domain $K \cap D$ (see Fig. 2) yields

$$
\int_{K \cap D} \nabla \cdot \boldsymbol{\omega}_{K}=\sum_{\mathfrak{s} \in \mathscr{E} D} \int_{K \cap \mathfrak{s}} \boldsymbol{\omega}_{K} \cdot \mathbf{n}_{D, \mathfrak{s}}+\sum_{\sigma \in \mathscr{E} K} \int_{D ̊} \boldsymbol{\omega}_{K} \cdot \mathbf{n}_{K, \sigma} .
$$

Note that in the second term of the right hand side, we exclude the edges $\sigma \in \mathscr{E}^{K}$ embedded in $\partial D$ since they are already taken into account in the first sum. This situation (see Fig. 2 right) cannot occur in the interior of the domain since $\mathfrak{T}$ and $\mathfrak{D}$ satisfy Assumption 2.6 but it may occur on the boundary of the domain. Note also that Assumption 2.6 is important here since it excludes the case where $K \cap D$ is not a polygonal domain but an union of segments. Owing to (5), the left hand side can be cast as

$$
\int_{K \cap D} \nabla \cdot \omega_{K}=|K \cap D| \operatorname{div}^{K}
$$

Since $\left\{\bar{D} \cap \sigma ; \sigma \in \mathscr{E}^{K}\right\}=\left\{\sigma^{D} \in \mathfrak{E}_{\mathfrak{D}}^{K} ; \sigma^{D} \subset D, \sigma^{D} \not \subset \partial D\right\}$, for all $K$, we arrive at

$$
|K \cap D| \operatorname{div}^{K}=\sum_{\mathfrak{s} \in \mathscr{E}^{D}} \int_{K \cap \mathfrak{s}} \boldsymbol{\omega}_{K} \cdot \mathbf{n}_{D, \mathfrak{s}}+\sum_{\substack{\sigma^{D} \in \mathfrak{E}_{\mathfrak{\Re}}^{K} \\ \sigma^{D} \subset D, \sigma^{D} \not \subset \partial D}} \int_{\sigma^{D}} \boldsymbol{\omega}_{K} \cdot \mathbf{n}_{K, \sigma^{D}}, \quad \forall K \in \mathfrak{T} .
$$

Summing these equalities for $K \in \mathfrak{T}$, we find

$$
\sum_{K \in \mathfrak{T}}|K \cap D| \operatorname{div}^{K}=\sum_{K \in \mathfrak{T}} \sum_{\mathfrak{s} \in \mathscr{E} D} \int_{K \cap \mathfrak{s}} \boldsymbol{\omega}_{K} \cdot \mathbf{n}_{D, \mathfrak{s}}+\sum_{K \in \mathfrak{T}} \sum_{\substack{\sigma^{D} \in \mathfrak{E}_{\mathfrak{Q}}^{K} \\ \sigma^{D} \subset D, \sigma^{D} \not \supset \partial D}} F_{K, \sigma^{D}} .
$$

The conclusion is obtained by inverting the two sums in the first term of the right hand side

$$
\begin{aligned}
\sum_{K \in \mathfrak{T}} \sum_{\mathfrak{s} \in \mathscr{E} D} \int_{K \cap \mathfrak{s}} \boldsymbol{\omega}_{K} \cdot \mathbf{n}_{D, \mathfrak{s}} & =\sum_{\mathfrak{s} \in \mathscr{E} D} \sum_{K \in \mathfrak{T}} \int_{K \cap \mathfrak{s}} \boldsymbol{\omega}_{K} \cdot \mathbf{n}_{D, \mathfrak{s}} \\
& =\sum_{\mathfrak{s} \in \mathscr{E} D} F_{D, \mathfrak{s}}
\end{aligned}
$$

Remark 2.8. The following comments are worthwhile: 

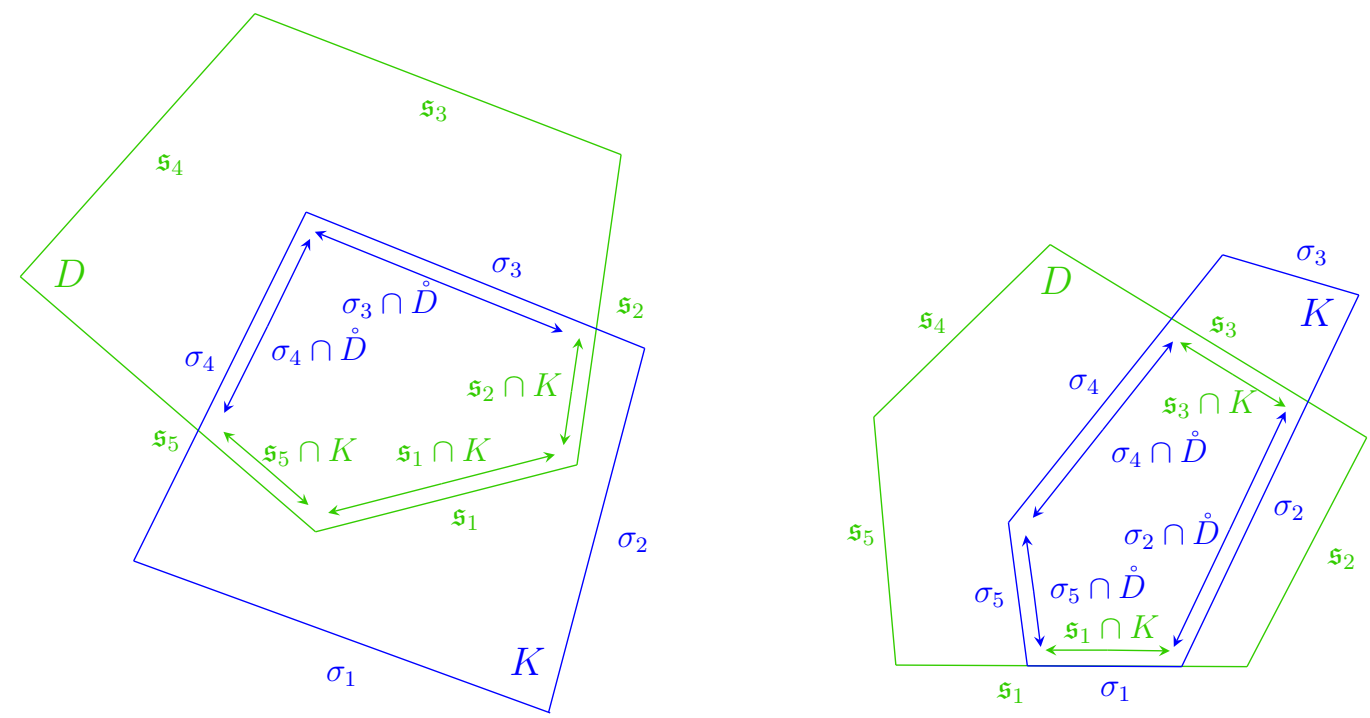

Figure 2: Two examples of intersection $D \cap K$ between a cell $D \in \mathfrak{D}$ and a cell $K \in \mathfrak{T}$.

- in Theorem 2.7, we do not assume the conservativity of the fluxes $F_{K, \sigma^{D}}$ or fluxes $F_{K, \sigma}$ but the resulting fluxes $F_{D, \mathfrak{s}}$ are conservative.

- this conservatity requirement (or even an additional consistency property) is not enough to ensure the uniqueness of fluxes $F_{D, \mathfrak{s}}$ in Theorem 2.7. Indeed, adding the same quantity (possibly proportional to the mesh size) to all fluxes going through the edges which share a given vertex does not modify the final result.

- in the second sum in the right hand side of (4), the edges $\sigma^{D}$ embedded in $\partial D$ are excluded. As a matter of fact, this implies that this sum does not contain fluxes through the boundary of the domain $\partial \Omega$. The boundary conditions are included in the operators $\operatorname{div}^{K}$ and $\operatorname{div}^{D}$.

- in practice, if we have only at hand the fluxes $F_{K, \sigma}$ associated to the entire edge $\sigma \in \mathscr{E}^{K}$, it is possible to apply Theorem 2.7 by simply setting

$$
F_{K, \sigma^{D}}=\frac{|\sigma \cap D|}{|\sigma|} F_{K, \sigma}, \quad \forall D \in \mathfrak{D}^{\sigma} .
$$

Then, the conclusion of Theorem 2.7 can be cast as

$$
|D| \operatorname{div}^{D}=\sum_{K \in \mathfrak{T}}|K \cap D| \operatorname{div}^{K}-\sum_{K \in \mathfrak{T}} \sum_{\sigma \in \mathscr{E}^{K}} \frac{|\sigma \cap \stackrel{\circ}{ }|}{|\sigma|} F_{K, \sigma}, \quad \forall D \in \mathfrak{D} .
$$

\subsection{Practical construction of the average operator}

In this section, we detail practical constructions of the transfer fluxes $F_{D, \mathfrak{s}}$ that can replace those constructed in Theorem 2.7. Since we have in mind applications to time 
dependent problems in fluid mechanics, where the transfer should be proceeded at each time step, it is crucial to design a construction which remains computationally affordable.

In the proof of Theorem 2.7, we note that the transfer fluxes are entirely determined by the function $\boldsymbol{\omega}_{K}$ having the properties required in the statement of Lemma 2.2, see (6). We thus propose in Section 2.2.1, several practical constructions of this function depending on the geometry of the considered cell $K$. There exist nevertheless several situations, of practical interest, in which the transfer fluxes $F_{D, \mathfrak{s}}$ can be directly expressed as linear combination of $F_{K, \sigma}$. We detail the case of quadrangular cells in Section 2.2.2.

\subsubsection{Construction of the function $\omega_{K}$}

In this Section, we are going to detail several ways to define the function $\boldsymbol{\omega}_{K}$ whose existence is ensured by Lemma 2.2. These constructions thus provide alternative proofs of this statement in the restricted situations where they apply, and with regularity properties that depend on the framework. Thus, we assume to have at hand a polygonal domain $K$ and fluxes $F_{K, \sigma}$ associated to each edge $\sigma \in \mathscr{E}^{K}$. We look for a function $\boldsymbol{\omega}_{K} \in H_{\text {div }}(\stackrel{\circ}{K})$ such that $\boldsymbol{\nabla} \cdot \boldsymbol{\omega}_{K}$ is constant and

$$
\int_{\sigma} \boldsymbol{\omega}_{K} \cdot \mathbf{n}_{K, \sigma}=F_{K, \sigma}, \quad \forall \sigma \in \mathscr{E}^{K} .
$$

Our constructions rely on the definition of piecewise polynomial functions reminiscent of finite element approximation spaces based on a convenient tessellation of $K$. Since we will use $\mathbb{P}^{1}$ polynomial function it is useful to associate to flux-quantities, stored on the edges, a vector field which is associated to the vertices in the following way.

Lemma 2.9. Let $K$ be a polygon in $\mathbb{R}^{2}$ with $n$ vertices denoted $P_{1}, \ldots, P_{n}$. For all $\sigma \in \mathscr{E}^{K}$, we consider a given quantity $F_{K, \sigma}$. There exist vector quantities $\mathbf{w}_{i} \in \mathbb{R}^{2}$, $i=1, . ., n$ associated to the vertices of $K$ such that, for all $\sigma \in \mathscr{E}^{K}$,

$$
\frac{\mathbf{w}_{i}+\mathbf{w}_{j}}{2} \cdot \mathbf{n}_{K, \sigma}=\frac{1}{|\sigma|} F_{K, \sigma},
$$

where $i$ and $j$ are the two indexes of $P_{i}$ and $P_{j}$ the vertices of $\sigma=\left[P_{i}, P_{j}\right]$.

For the sake of completeness, the proof of this Lemma is detailed in Appendix B. This statement is useful for the first two constructions presented below. Further we observe that, if $\boldsymbol{\omega}_{K}$ is $\mathbb{P}^{1}$ on $\sigma \in \mathscr{E}^{K}$, then we have

$$
\begin{aligned}
\int_{\sigma} \boldsymbol{\omega}_{K} \cdot \mathbf{n}_{K, \sigma} & =|\sigma| \boldsymbol{\omega}_{K}\left(\frac{P_{i}+P_{j}}{2}\right) \cdot \mathbf{n}_{K, \sigma}, \\
& =|\sigma| \frac{\boldsymbol{\omega}_{K}\left(P_{i}\right)+\boldsymbol{\omega}_{K}\left(P_{j}\right)}{2} \cdot \mathbf{n}_{K, \sigma},
\end{aligned}
$$

where $P_{i}$ and $P_{j}$ stand for the vertices of $\sigma=\left[P_{i}, P_{j}\right]$. Thus, in the case where we look for a piecewise $\mathbb{P}^{1}$ function $\boldsymbol{\omega}_{K}$, denoting by $\mathbf{w}_{i}$ the vectors provided by Lemma 2.9 from $F_{K, \sigma}$, we observe that it is sufficient to ensure that

$$
\boldsymbol{\omega}_{K}\left(P_{i}\right)=\mathbf{w}_{i}, \quad \forall i \in\{1, . ., n\},
$$


to guarantee that (7) holds. A key (but basic) observation is the fact that if $\boldsymbol{\omega}_{K}$ has a constant divergence over a domain $K$ and it satisfies (7), then the value of the divergence is automatically given by $\nabla \cdot \boldsymbol{\omega}_{K}=\frac{1}{|K|} \sum_{\sigma \in \mathscr{E}_{K}} F_{K, \sigma}$ since

$$
\begin{aligned}
\int_{K} \nabla \cdot \boldsymbol{\omega}_{K} & =|K| \nabla \cdot \boldsymbol{\omega}_{K} & & \text { since } \nabla \cdot \boldsymbol{\omega}_{K} \text { is constant over } K \\
& =\sum_{\sigma \in \mathscr{E}^{K}} \int_{\sigma} \boldsymbol{\omega}_{K} \cdot \mathbf{n}_{K, \sigma} & & \text { by Green's formula } \\
& =\sum_{\sigma \in \mathscr{E}^{K}} F_{K, \sigma} & & \text { by }(7) .
\end{aligned}
$$

Triangular domains: the $\mathbb{P}^{1}$ approach As a warm up, observe that when $K$ is a triangle, we readily find a $\mathbb{P}^{1}$ function $\boldsymbol{\omega}_{K}$ (ensuring that $\boldsymbol{\nabla} \cdot \boldsymbol{\omega}_{K}$ constant) which fulfils (8). Let $P_{1}, P_{2}, P_{3}$ be the vertices of $K$. Let $\phi_{1}, \phi_{2}, \phi_{3}$ be the $\mathbb{P}^{1}$ interpolation functions defined by $\phi_{i}\left(P_{j}\right)=\delta_{i, j}$. We simply set $\boldsymbol{\omega}_{K}=\sum_{i=1}^{3} \mathbf{w}_{i} \phi_{i}$ where $\mathbf{w}_{i}$ are the vectors provided by Lemma 2.9 from $F_{K, \sigma}$. It clearly satisfies $\boldsymbol{\omega}_{K}\left(P_{i}\right)=\mathbf{w}_{i}$.

Quadrilateral domains: the Nagtegaal approach The case of quadrilateral and convex cells is investigated in [17]; in this specific case, the proof is reminiscent of the construction of the Nagtegaal finite element [24]. Let $P_{1}, P_{2}, P_{3}, P_{4}$ be the four vertices of $K$. We are going to prove the existence of a piecewise $\mathbb{P}^{1}$ function $\boldsymbol{\omega}_{K}$ belonging to $H^{1}(\stackrel{\circ}{K})$, satifying $(8)$ and such that $\boldsymbol{\nabla} \cdot \boldsymbol{\omega}_{K}$ is constant. We denote by $P_{0}$ the intersection of the diagonals $\left[P_{1}, P_{3}\right]$ and $\left[P_{2}, P_{4}\right]$, which defines a subdivision of $K$ into four triangles $T_{1,2}, T_{2,3}, T_{3,4}$ and $T_{4,1}$ see Fig. 3 . Let $\phi_{0}, \ldots, \phi_{4}$ be the usual $\mathbb{P}^{1}$ Lagrange basis on $K$, defined by the points $P_{0}, \ldots, P_{4}$ : the functions $\phi_{k}$ 's are $\mathbb{P}^{1}$ on each triangles $T_{i, j}$ in Fig. 3, and satisfy $\phi_{i}\left(P_{j}\right)=\delta_{i j}$, for all $i, j=0, . ., 4$. For any piecewise $\mathbb{P}^{1}$ function $p$, we have $p=\sum_{i=0}^{4} p\left(P_{i}\right) \phi_{i}$. In particular, we have

$$
\sum_{i=0}^{4} \phi_{i}=1
$$

Further useful details about the gradient of the basis functions $\phi_{k}$ can be found in Appendix C.

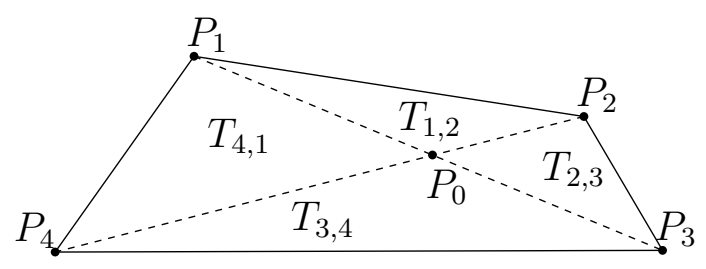

Figure 3: Case of a convex quadrangle $K$.

We turn to the determination of the vector field $\boldsymbol{\omega}_{K}$ under the form

$$
\boldsymbol{\omega}_{K}:(x, y) \in K \longmapsto \boldsymbol{\omega}(x, y)=\sum_{i=0}^{4} \mathbf{w}_{i} \phi_{i}(x, y),
$$


which is $\mathbb{P}^{1}$ on each subdomain $T_{i, j}$ (see Fig. 3 ) and belongs to $H^{1}(\stackrel{\circ}{K})$. The coefficients $\mathbf{w}_{1}, \ldots, \mathbf{w}_{4}$ are provided by Lemma 2.9 from $F_{K, \sigma}$, so that (8) holds, and we seek $\mathbf{w}_{0}$ such that $\boldsymbol{\nabla} \cdot \boldsymbol{\omega}_{K}$ is constant on $K$. Owing to (9), we write

$$
\boldsymbol{\omega}_{K}=\sum_{i=1}^{4} \mathbf{w}_{i} \phi_{i}+\mathbf{w}_{0}\left(1-\sum_{i=1}^{4} \phi_{i}\right),
$$

and thus

$$
\boldsymbol{\nabla} \cdot \boldsymbol{\omega}_{K}=\sum_{i=1}^{4} \mathbf{w}_{i} \cdot \boldsymbol{\nabla} \phi_{i}-\mathbf{w}_{0} \cdot \sum_{i=1}^{4} \boldsymbol{\nabla} \phi_{i} .
$$

This leads to four equations, one for each triangle $T_{j, k}$ :

$$
\left\{\begin{array}{l}
\left(e_{T_{1,2}}\right): \boldsymbol{\nabla} \cdot \boldsymbol{\omega}_{K}=\mathbf{w}_{1} \cdot \boldsymbol{\nabla} \phi_{1}+\mathbf{w}_{2} \cdot \boldsymbol{\nabla} \phi_{2}-\mathbf{w}_{0} \cdot \boldsymbol{\nabla}\left(\phi_{1}+\phi_{2}\right), \\
\left(e_{T_{2,3}}\right): \boldsymbol{\nabla} \cdot \boldsymbol{\omega}_{K}=\mathbf{w}_{2} \cdot \boldsymbol{\nabla} \phi_{2}+\mathbf{w}_{3} \cdot \boldsymbol{\nabla} \phi_{3}-\mathbf{w}_{0} \cdot \boldsymbol{\nabla}\left(\phi_{2}+\phi_{3}\right), \\
\left(e_{T_{3,4}}\right): \boldsymbol{\nabla} \cdot \boldsymbol{\omega}_{K}=\mathbf{w}_{3} \cdot \boldsymbol{\nabla} \phi_{3}+\mathbf{w}_{4} \cdot \boldsymbol{\nabla} \phi_{4}-\mathbf{w}_{0} \cdot \boldsymbol{\nabla}\left(\phi_{3}+\phi_{4}\right), \\
\left(e_{T_{4,1}}\right): \boldsymbol{\nabla} \cdot \boldsymbol{\omega}_{K}=\mathbf{w}_{4} \cdot \boldsymbol{\nabla} \phi_{4}+\mathbf{w}_{1} \cdot \boldsymbol{\nabla} \phi_{1}-\mathbf{w}_{0} \cdot \boldsymbol{\nabla}\left(\phi_{4}+\phi_{1}\right) .
\end{array}\right.
$$

We bear in mind that not only $\boldsymbol{\nabla} \phi_{i}$ is constant over each of these triangles, but, furthermore, due to the specific geometric configuration, since $P_{4}, P_{0}$ and $P_{2}$ are aligned, $\boldsymbol{\nabla} \phi_{1}$ is the same in $T_{1,2}$ and $T_{4,1}$ (see Appendix C). Similarly, since $P_{1}$, $P_{0}$ and $P_{3}$ are aligned, $\boldsymbol{\nabla} \phi_{2}$ is the same in $T_{1,2}$ and $T_{2,3}$, etc. We thus remark that $\left(e_{T_{1,2}}\right)-\left(e_{T_{2,3}}\right)+\left(e_{T_{3,4}}\right)-\left(e_{T_{4,1}}\right)=0$. We deduce from this equality that $\boldsymbol{\nabla} \cdot \boldsymbol{\omega}_{K}$ takes a single value over $K$ iff we have on the one hand $\left(e_{T_{1,2}}\right)-\left(e_{T_{2,3}}\right)=0$ and on the other hand $\left(e_{T_{2,3}}\right)-\left(e_{T_{3,4}}\right)=0$, that is, in other words,

$$
\left\{\begin{array}{l}
\mathbf{w}_{1} \cdot \nabla \phi_{1}-\mathbf{w}_{3} \cdot \nabla \phi_{3}=\mathbf{w}_{0} \cdot \nabla\left(\phi_{1}-\phi_{3}\right), \\
\mathbf{w}_{2} \cdot \nabla \phi_{2}-\mathbf{w}_{4} \cdot \nabla \phi_{4}=\mathbf{w}_{0} \cdot \nabla\left(\phi_{2}-\phi_{4}\right) .
\end{array}\right.
$$

This is a linear system of two equations for the components of $\mathbf{w}_{0}$ and we are wondering whether or not it is invertible. We know that $\boldsymbol{\nabla} \phi_{1}$ and $\boldsymbol{\nabla} \phi_{3}$ are both colinear to $\left[P_{2}, P_{4}\right]$ but of opposite sign (see Appendix C), so that $\nabla\left(\phi_{1}-\phi_{3}\right) \neq 0$. Similarly, $\boldsymbol{\nabla}\left(\phi_{2}-\phi_{4}\right) \neq 0$ is colinear to $\left[P_{1}, P_{3}\right]$. Therefore $\boldsymbol{\nabla}\left(\phi_{1}-\phi_{3}\right)$ and $\boldsymbol{\nabla}\left(\phi_{2}-\phi_{4}\right)$ are linearly independent which implies that the system (11) admits a unique solution. It determines $\mathbf{w}_{0}$. Eventually, we have found a piecewise $\mathbb{P}^{1}$ function $\boldsymbol{\omega}_{K} \in H^{1}(\stackrel{\circ}{K})$ such that (8) holds and $\boldsymbol{\nabla} \cdot \boldsymbol{\omega}_{K}$ is constant on $K$.

\section{Star-shaped polygonal domains: the Raviart-Thomas $\left(R T_{0}\right)$ approach} In this section, we consider that $K$ is a star-shaped polygonal domain. We denote by $n$ the number of edges of $K$. The edges $\sigma_{i}, i \in 1, \ldots, n$, are labelled by browsing $\partial K$ clockwise (see Fig. 4). At each edge $\sigma_{i}$, it is associated the given flux $F_{K, \sigma_{i}}$. We pick a point in the domain so that joining the vertices to this point (this is where we use the star-shaped assumption) we construct a tessellation of the domain by $n$ triangles. We denote by $T_{i}, i \in\{1, \ldots, n\}$, these triangles (with $\sigma_{i} \subset \partial T_{i}$ ) and $t_{i}=\left|T_{i}\right|, i \in\{1, \ldots, n\}$, the volume of $T_{i}$. 


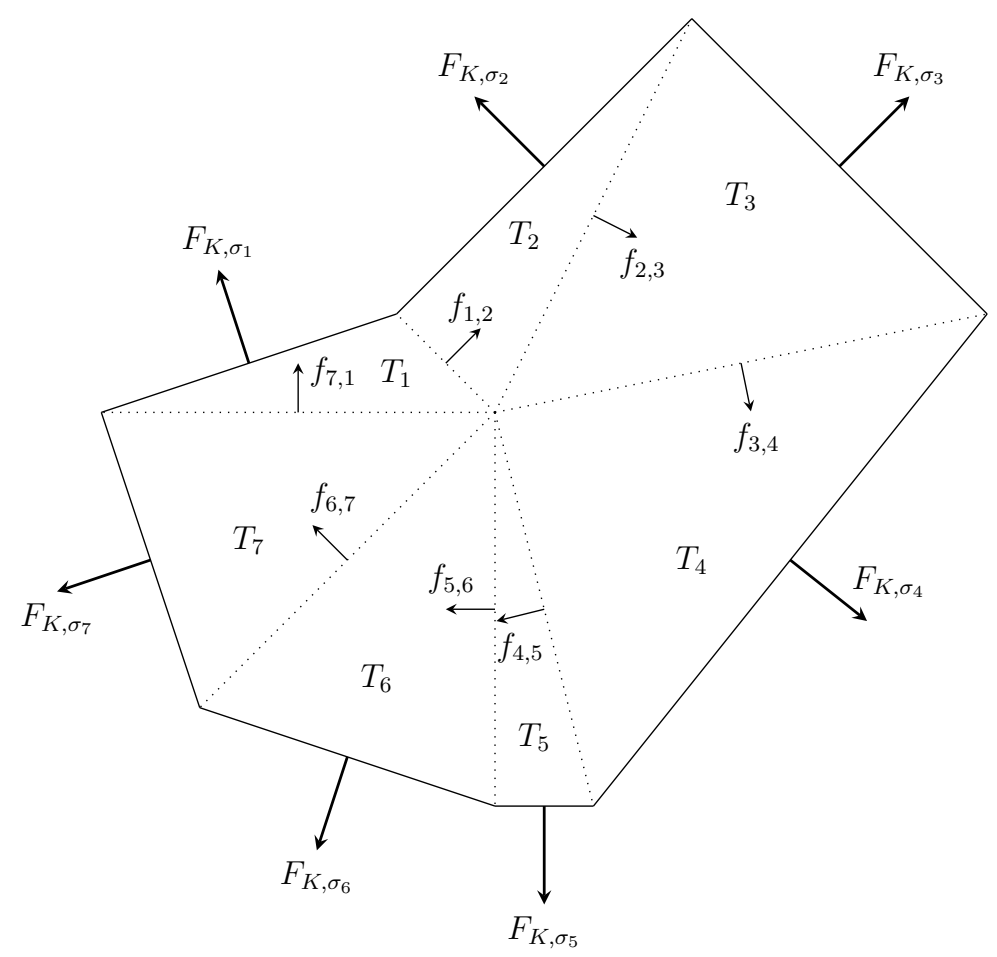

Figure 4: Construction of the auxiliary fluxes

We wish to define fluxes $f_{i, i+1}, i \in\{1, \ldots, n\}$ from $T_{i}$ to $T_{i+1}$ on the interfaces between these triangles with the convention that $f_{n, n+1}=f_{n, 1}$ stands for the flux from $T_{n}$ to $T_{1}$. We consider these fluxes as defining an $R T_{0}$ function $\boldsymbol{\omega}_{K}$ [25] over the triangular tessellation of $K$. Over the triangle $T_{i}$, the divergence of $\boldsymbol{\omega}_{K}$ is constant, and we get

$$
\int_{T_{i}} \boldsymbol{\nabla} \cdot\left(\boldsymbol{\omega}_{K \mid T_{i}}\right)=t_{i} \boldsymbol{\nabla} \cdot\left(\boldsymbol{\omega}_{K \mid T_{i}}\right)=F_{K, \sigma_{i}}+f_{i, i+1}-f_{i-1, i}
$$

Accordingly, the divergence of $\boldsymbol{\omega}_{K}$ on the triangle $T_{i}$ reads

$$
\boldsymbol{\nabla} \cdot\left(\boldsymbol{\omega}_{K \mid T_{i}}\right)=\frac{1}{t_{i}}\left(F_{K, \sigma_{i}}+f_{i, i+1}-f_{i-1, i}\right) .
$$

We wish to define the internal fluxes $f_{i, i+1}$ by requesting that this quantity remains 
constant over all the domain. It leads to the following linear system

$$
\begin{aligned}
\left(\begin{array}{ccccccc}
t_{1}+t_{2} & -t_{1} & 0 & & \cdots & 0 & -t_{2} \\
-t_{3} & t_{2}+t_{3} & -t_{2} & 0 & \cdots & 0 & 0 \\
-t_{n} & 0 & \cdots & 0 & 0 & -t_{1} & t_{1}+t_{n}
\end{array}\right)\left(\begin{array}{c}
f_{1,2} \\
f_{2,3} \\
\vdots \\
f_{n, 1}
\end{array}\right) \\
=\left(\begin{array}{cccccc}
-t_{2} & t_{1} & 0 & 0 & \cdots & 0 \\
0 & -t_{3} & t_{2} & 0 & \cdots & 0 \\
& & & & & \\
t_{n} & 0 & 0 & \cdots & 0 & -t_{1}
\end{array}\right)\left(\begin{array}{c}
F_{K, \sigma_{1}} \\
F_{K, \sigma_{2}} \\
\vdots \\
F_{K, \sigma_{n}}
\end{array}\right),
\end{aligned}
$$

that we denote $A f=B F$.

Let $e$ be the vector in $\mathbb{R}^{n}$ with all components equal to 1 . We claim that $\operatorname{Ker}(A)=$ $\operatorname{Span}(e)$. It is clear that $A e=0$. Now, let $u$ be such that $A u=0$ and consider $i \in\{1, \ldots, n\}$ such that $u_{i}=\min \left\{u_{j}, j \in\{1, \ldots n\}\right\}$. We have

$$
t_{i} \underbrace{\left(u_{i}-u_{i+1}\right)}_{\leqslant 0}+t_{i+1} \underbrace{\left(u_{i}-u_{i-1}\right)}_{\leqslant 0}=0,
$$

which imposes $u_{i}=u_{i+1}=u_{i-1}$. Repeating this process with $i-1$ instead of $i$ and so on, we conclude that $u$ is colinear to $e$. It follows that $\operatorname{Ker}\left(A^{\top}\right)$ has dimension 1 and we check that it is spanned by $u$ the vector whose components are given by

$$
u_{i}=t_{1} \ldots t_{i-1} t_{i+2} \ldots t_{n} .
$$

We moreover observe that $B^{\top} u=0$, so that for any $F \in \mathbb{R}^{n}, B F$ belongs to $\left(\operatorname{Ker}\left(A^{\top}\right)\right)^{\perp}=$ $\operatorname{Ran}(A)$. We conclude that the system $A f=B F$ admits a solution, which can be uniquely defined by imposing for instance that it is also orthogonal to $e$. Thus, by construction, the function $\boldsymbol{\omega}_{K}$ belongs to $H_{\text {div }}(\stackrel{\circ}{K})$, satisfies $(7)$ and $\boldsymbol{\nabla} \cdot \boldsymbol{\omega}_{K}$ is constant over the domain $K$.

As pointed out by one of the reviewers, the solution selected by the criterion

$$
\sum_{i=1}^{n} f_{i, i+1}=0
$$

might be not the most relevant. It is possible to improve the consistency of this construction. Indeed, for any constant $c$ (independent of $i$ )

$$
\tilde{f}_{i, i+1}=f_{i, i+1}+c
$$

still provides a solution of the linear system. We define this constant from the set of the $F_{K, \sigma}$ 's by setting

$$
c=\sum_{i=1}^{n} P_{0} P_{i, i+1}^{\perp} \cdot \mathbf{v}, \quad \mathbf{v}=\frac{1}{|K|} \sum_{i=1}^{n} F_{K, \sigma_{i}}\left(P_{\sigma_{i}}-P_{0}\right)
$$

where $P_{\sigma_{i}}$ is the midpoint of the edge $\sigma_{i}$ and $P_{i, i+1}$ is the vertex of $K$ common to $\sigma_{i}$ and $\sigma_{i+1}$. Accordingly, the modified solution is such that $\sum_{i=1}^{n} \tilde{f}_{i, i+1}=n c$. It satisfies 
an additional relevant consistency property. Indeed, in the specific case of a velocity field a constant over the domain, it turns out that

$$
\frac{1}{|K|} \sum_{i=1}^{n} F_{K, \sigma_{i}}\left(P_{\sigma_{i}}-P_{0}\right)=\sum_{i=1}^{4} \frac{\left|\sigma_{i}\right|}{|K|} \mathbf{a} \cdot \mathbf{n}_{K, \sigma_{i}}\left(P_{\sigma_{i}}-P_{0}\right)=\mathbf{a}
$$

as remarked in [12] or [6, Lemma 7.1]. This statement is a direct consequence of Green's formula applied to $P \in K \mapsto\left(P-P_{0}\right)_{j}$ a for $j \in 1,2$. If we then consider the fluxes $F_{K, \sigma}=|\sigma| \mathbf{a} \cdot \mathbf{n}_{K, \sigma}$, we remark that the modified fluxes are actually given by the natural formula

$$
\tilde{f}_{i, i+1}=P_{0} P_{i, i+1}^{\perp} \cdot \mathbf{a} .
$$

Indeed, coming back to (12) the rows of $A \tilde{f}$ read

$$
\left(t_{i}+t_{i+1}\right) \tilde{f}_{i, i+1}-t_{i} \tilde{f}_{i+1, i+2}-t_{i+1} \tilde{f}_{i-1, i}=t_{i}\left(\tilde{f}_{i, i+1}-\tilde{f}_{i+1, i+2}\right)+t_{i+1}\left(\tilde{f}_{i, i+1}-\tilde{f}_{i-1, i}\right) .
$$

With $\tilde{f}_{i, i+1}=P_{0} P_{i, i+1}^{\perp} \cdot \mathbf{a}$, it becomes

$$
\begin{aligned}
\left(t_{i}\left(P_{0} P_{i, i+1}^{\perp}-P_{0} P_{i+1, i+2}^{\perp}\right)+t_{i+1}\left(P_{0} P_{i, i+1}^{\perp}\right.\right. & \left.\left.-P_{0} P_{i-1, i}^{\perp}\right)\right) \cdot \mathbf{a} \\
& =\left(t_{i}\left(\left|\sigma_{i+1}\right| \mathbf{n}_{K, \sigma_{i}+1}-t_{i+1}\left|\sigma_{i}\right| \mathbf{n}_{K, \sigma_{i}}\right) \cdot \mathbf{a},\right.
\end{aligned}
$$

by virtue of Lemma 2.1. It coincides with the expression of the rows of $B F$. Hence, the construction provided by (12)-(14) is interesting for the purposes of Theorem 2.7 since the consistency properties of the original fluxes $F_{K, \sigma^{D}}$ (in the sense that $F_{K, \sigma^{D}}$ tends to $\int_{\sigma^{D}} \mathbf{a} \cdot \mathbf{n}_{K, \sigma^{D}}$ for some velocity field $\mathbf{a}$ as the mesh is refined) are inherited by the new fluxes $F_{D, \mathfrak{s}}$.

This discussion also gives the opportunity to further comment the role of the auxiliary function $\omega_{K}$ in order to construct relevant balance on subcells of $K$. Indeed, different choices of the function $\omega_{K}$ will lead to different definitions of fluxes $F_{D, \mathfrak{s}}$. However, it turns out that, in practice, a point that can play the role of $P_{0}$ in the construction above is often naturally available in each cell by the construction of the meshes. We get a tessellation of $K$ by joining all the vertices to $P_{0}$, as in Fig. 4 and the fluxes of interest are the fluxes through the interfaces $\sigma_{i, i+1}=T_{i} \mid T_{i+1}$. Given two functions $\omega_{K}$ and $\omega_{K}^{\prime}$, we define the fluxes on the interfaces $\sigma_{i, i+1}$ by $f_{i, i+1}=\int_{\sigma_{i, i+1}} \omega_{K} \cdot \mathbf{n}_{K, \sigma_{i, i+1}}$ and $f_{i, i+1}^{\prime}=\int_{\sigma_{i, i+1}} \omega_{K}^{\prime} \cdot \mathbf{n}_{K, \sigma_{i, i+1}}$. By definition both satisfy the linear system (12) and thus, for any $i, f_{i, i+1}=f_{i, i+1}^{\prime}+c$, for some constant $c$. Thus, the fluxes obtained from $\omega_{K}$ and those obtained from $\omega_{K}^{\prime}$ differ only from a constant.

Moreover, when performing a balance over $T_{i} \cup T_{i+1} \cup \ldots \cup T_{j} \subset K$, it makes the following quantity appear

$$
f_{i, i+1}-f_{j, j+1}=\frac{t_{i}+\ldots+t_{j}}{|K|} \sum_{m=1}^{n} F_{K, \sigma_{m}}-\sum_{k=i}^{j} F_{K, \sigma_{k}}
$$

which does not depend on $\omega_{K}$. It turns out that, for our purposes, we only need to deal with such combinations, which thus do not depend on the details of the function $\omega_{K}$. 


\subsubsection{Expression of the transfer fluxes for quadrangular cells}

We focus here on the specific case of quadrangles (convex or not). Quadrangular meshes are often used in practice and, furthermore, they can also be encountered in unstructured methods (e.g. DDFV methods [17]) which make use of diamond meshes which are always constituted of quadrangles.

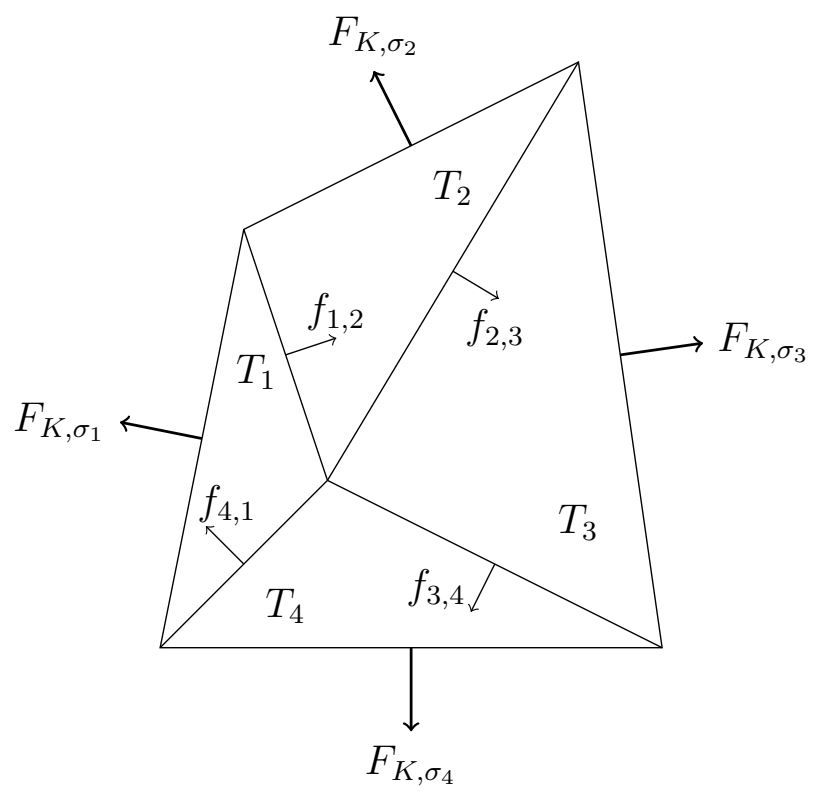

Figure 5: Spectific case of quadrangle

In these cases, a point lies in the quadrangle and we have to compute the transfer fluxes through the lines joining this point to the vertices of the quadrangle, the fluxes through the edges of the quadrangle being known (see Fig. 5). In the case of quadrangular meshes, the interior lines often correspond to the edges of a diamond mesh (see e.g. $[3,5])$ whereas in the case of the diamond meshes it corresponds to the edges of the primal or the dual mesh (as in the DDFV framework [17], see also Section 3.3.2). It turns out that the corresponding linear system, as described in the previous section, can be solved by hands.

Using the same notation as in the previous section, we know the boundary fluxes $F_{K, \sigma_{i}}, i=1, . ., 4$ and we aim at defining the interior fluxes $f_{1,2}, f_{2,3}, f_{3,4}$ and $f_{4,1}$. It is not necessary to give the expression of the function $\boldsymbol{\omega}_{K}$ in this case, we obtain directly the expression of the transfer fluxes by solving the linear system (12). We obtain the following formula with $t_{1}, t_{2}, t_{3}$ and $t_{4}$ the volumes of the four triangles $T_{i}$ splitting $K$ :

$$
\begin{aligned}
f_{1,2}=\frac{1}{4\left(t_{1}+t_{2}+t_{3}+t_{4}\right)}\left[-\left(3 t_{2}+\right.\right. & \left.2 t_{3}+t_{4}\right) F_{K, \sigma_{1}}+\left(3 t_{1}+t_{3}+2 t_{4}\right) F_{K, \sigma_{2}} \\
& \left.+\left(2 t_{1}-t_{2}+t_{4}\right) F_{K, \sigma_{3}}-\left(-t_{1}+2 t_{2}+t_{3}\right) F_{K, \sigma_{4}}\right]
\end{aligned}
$$




$$
\begin{aligned}
& f_{2,3}=\frac{1}{4\left(t_{1}+t_{2}+t_{3}+t_{4}\right)}\left[-\left(-t_{2}+2 t_{3}+t_{4}\right) F_{K, \sigma_{1}}-\left(t_{1}+3 t_{3}+2 t_{4}\right) F_{K, \sigma_{2}}\right. \\
& \left.+\left(2 t_{1}+3 t_{2}+t_{4}\right) F_{K, \sigma_{3}}+\left(t_{1}+2 t_{2}-t_{3}\right) F_{K, \sigma_{4}}\right] \\
& f_{3,4}=\frac{1}{4\left(t_{1}+t_{2}+t_{3}+t_{4}\right)}\left[\left(t_{2}+2 t_{3}-t_{4}\right) F_{K, \sigma_{1}}-\left(t_{1}-t_{3}+2 t_{4}\right) F_{K, \sigma_{2}}\right. \\
& \left.-\left(2 t_{1}+t_{2}+3 t_{4}\right) F_{K, \sigma_{3}}+\left(t_{1}+2 t_{2}+3 t_{3}\right) F_{K, \sigma_{4}}\right] \\
& f_{4,1}=\frac{1}{4\left(t_{1}+t_{2}+t_{3}+t_{4}\right)}\left[\left(t_{2}+2 t_{3}+3 t_{4}\right) F_{K, \sigma_{1}}+\left(-t_{1}+t_{3}+2 t_{4}\right) F_{K, \sigma_{2}}\right. \\
& \left.-\left(2 t_{1}+t_{2}-t_{4}\right) F_{K, \sigma_{3}}-\left(3 t_{1}+2 t_{2}+t_{3}\right) F_{K, \sigma_{4}}\right]
\end{aligned}
$$

These expressions allow us to recover formula presented in previous works. If we assume that the volumes of the four triangles are equal (that is $t_{1}=t_{2}=t_{3}=t_{4}$ ) we obtain

$$
\begin{aligned}
& f_{1,2}=-\frac{3}{8} F_{K, \sigma_{1}}+\frac{3}{8} F_{K, \sigma_{2}}+\frac{1}{8} F_{K, \sigma_{3}}-\frac{1}{8} F_{K, \sigma_{4}} \\
& f_{2,3}=-\frac{1}{8} F_{K, \sigma_{1}}-\frac{3}{8} F_{K, \sigma_{2}}+\frac{3}{8} F_{K, \sigma_{3}}+\frac{1}{8} F_{K, \sigma_{4}} \\
& f_{3,4}=\frac{1}{8} F_{K, \sigma_{1}}-\frac{1}{8} F_{K, \sigma_{2}}-\frac{3}{8} F_{K, \sigma_{3}}+\frac{3}{8} F_{K, \sigma_{4}} \\
& f_{4,1}=\frac{3}{8} F_{K, \sigma_{1}}+\frac{1}{8} F_{K, \sigma_{2}}-\frac{1}{8} F_{K, \sigma_{3}}-\frac{3}{8} F_{K, \sigma_{4}}
\end{aligned}
$$

This is exactly the solution proposed in $[3,5]$ when dealing with rectangles split by their diagonals.

When summing the fluxes along the lines joining two opposite vertices (and passing through the interior point) we obtain

$$
\begin{gathered}
f_{1,2}-f_{3,4}=\frac{t_{1}+t_{4}}{t_{1}+t_{2}+t_{3}+t_{4}}\left(F_{K, \sigma_{2}}+F_{K, \sigma_{3}}\right)-\frac{t_{2}+t_{3}}{t_{1}+t_{2}+t_{3}+t_{4}}\left(F_{K, \sigma_{1}}+F_{K, \sigma_{4}}\right) \\
f_{2,3}-f_{4,1}=-\frac{t_{3}+t_{4}}{t_{1}+t_{2}+t_{3}+t_{4}}\left(F_{K, \sigma_{1}}+F_{K, \sigma_{2}}\right)+\frac{t_{1}+t_{2}}{t_{1}+t_{2}+t_{3}+t_{4}}\left(F_{K, \sigma_{3}}+F_{K, \sigma_{4}}\right)
\end{gathered}
$$

In DDFV framework, it exactly corresponds to the formula presented in [17] to transfer the mass fluxes from diamond mesh to primal or dual meshes. In that case, the fluxes $f_{1,2}$ and $-f_{3,4}$ (resp. $f_{2,3}$ and $-f_{4,1}$ ) are going out the same cell, so that their sum (instead of their single values) is the quantity of interest. Note that this formula works even if the internal point is not the intersection of the diagonals (as it is the case when considering the barycentric dual mesh). Remarkably, as done in [17], this formula 
can also be obtained using the existence of the function $\boldsymbol{\omega}_{K}$ but not the details of its construction. Indeed, since $\boldsymbol{\nabla} \cdot \boldsymbol{\omega}_{K}=\left(F_{K, \sigma_{1}}+F_{K, \sigma_{2}}+F_{K, \sigma_{3}}+F_{K, \sigma_{4}}\right) /|K|$, a balance on $T_{2} \cup T_{3}$ gives, on the one hand

$$
\int_{T_{2} \cup T_{3}} \boldsymbol{\nabla} \cdot \boldsymbol{\omega}_{K}=\frac{t_{2}+t_{3}}{t_{1}+t_{2}+t_{3}+t_{4}}\left(F_{K, \sigma_{1}}+F_{K, \sigma_{2}}+F_{K, \sigma_{3}}+F_{K, \sigma_{4}}\right),
$$

and, on the other hand, by the Green formula

$$
\int_{T_{2} \cup T_{3}} \boldsymbol{\nabla} \cdot \boldsymbol{\omega}_{K}=-f_{1,2}+f_{3,4}+F_{K, \sigma_{2}}+F_{K, \sigma_{3}} .
$$

\section{Examples of applications}

\subsection{A simple but illuminating example}

Restricting to the one-dimensional framework makes the discussion completely elementary, but it illustrates our aims (we refer the reader to $[18,23]$ for applications to the analysis of schemes for the Euler equations). In this case in particular, the function $\omega$ is nothing but the affine function connecting two given values. Let $K=\left[x_{L}, x_{R}\right]$, with given fluxes $\mathcal{X}_{L}$, and $\mathcal{X}_{R}$ given at the end points. We have

$$
\omega(x)=\frac{x-x_{L}}{x_{R}-x_{L}}\left(\mathcal{X}_{R}-\mathcal{X}_{L}\right)+\mathcal{X}_{L},
$$

which indeed satisfies $\omega\left(x_{L}\right)=\mathcal{X}_{L}, \omega\left(x_{R}\right)=\mathcal{X}_{R}$ and $\omega^{\prime}(x)=\mathcal{X}_{R}-\mathcal{X}_{L}$.

In order to keep the discussion less cluttered, we neglect for the time being the difficulties related to boundaries, and we consider a set of points, denoted $x_{j+\frac{1}{2}}$ on the line. We denote $\mathscr{C}_{j}=\left[x_{j-\frac{1}{2}}, x_{j+\frac{1}{2}}\right]$ the primal cells, with cell centers $x_{j}=\left(x_{j-\frac{1}{2}}+\right.$ $\left.x_{j+\frac{1}{2}}\right) / 2$ and size $\delta x_{j}=x_{j+\frac{1}{2}}-x_{j-\frac{1}{2}}$. Next, we consider the dual cells $\mathscr{C}_{j+\frac{1}{2}}=\left[x_{j}, x_{j+1}\right]$, with size $\delta x_{j+\frac{1}{2}}=x_{j+1}-x_{j}$. We refer the reader to Fig. 6 for an illustration of this discretization. We deal with discrete quantities, stored on the two meshes, namely the $\rho_{j}$ 's are stored at the centers $x_{j}$ whereas the $u_{j+\frac{1}{2}}$ 's are located at the edges $x_{j+\frac{1}{2}}$. We define the discrete "gradient" and "divergence"

$$
(\nabla \rho)_{j+\frac{1}{2}}=\frac{\rho_{j+1}-\rho_{j}}{\delta x_{j+\frac{1}{2}}}, \quad(\operatorname{div} u)_{j}=\frac{u_{j+\frac{1}{2}}-u_{j-\frac{1}{2}}}{\delta x_{j}} .
$$

We wonder how discrete conservation laws can be transferred from a grid to another and what is the discrete analog of the formula

$$
\int_{a}^{b} u \partial_{x} \rho \mathrm{d} x+\int_{a}^{b} \rho \partial_{x} u \mathrm{~d} x=u(b)-u(a) .
$$

This question is ambiguous since the quantities $\rho$ and $u$, and the associated differential operators, are not defined on the same grid.

Let us suppose that the quantity $\rho$ is updated with the discrete conservation law

$$
\frac{\bar{\rho}_{j}-\rho_{j}}{\Delta t}+\frac{F_{j+\frac{1}{2}}-F_{j-\frac{1}{2}}}{\delta x_{j}}=0 .
$$


On the dual grid, we define

$$
\rho_{j+\frac{1}{2}}=\frac{\delta x_{j} \rho_{j}+\delta x_{j+1} \rho_{j+1}}{2 \delta x_{j+\frac{1}{2}}}
$$

This is the mean over $\mathscr{C}_{j+\frac{1}{2}}$ of the piecewise constant function $\rho(x)=\sum_{j} \rho_{j} \mathbf{1}_{\mathscr{C}_{j}}(x)$. We simply get

$$
\frac{\bar{\rho}_{j+\frac{1}{2}}-\rho_{j+\frac{1}{2}}}{\Delta t}+\frac{F_{j+1}-F_{j}}{\delta x_{j+\frac{1}{2}}}=0
$$

with

$$
F_{j}=\frac{F_{j+\frac{1}{2}}+F_{j-\frac{1}{2}}}{2} .
$$

It can be interpreted as $F_{j}=\omega\left(x_{j}\right)$ with

$$
\omega(x)=\frac{x-x_{j-\frac{1}{2}}}{\delta x_{i}}\left(F_{j+\frac{1}{2}}-F_{j-\frac{1}{2}}\right)+F_{j-\frac{1}{2}}
$$

since $x_{j}-x_{j-\frac{1}{2}}=\delta x_{j} / 2$. Similarly, let us consider the relation

$$
\frac{\bar{u}_{j+\frac{1}{2}}-u_{j+\frac{1}{2}}}{\Delta t}+\frac{G_{j+1}-G_{j}}{\delta x_{j+\frac{1}{2}}}=0 .
$$

We go back to the primal grid with

$$
u_{j}=\frac{u_{j+\frac{1}{2}}+u_{j-\frac{1}{2}}}{2} .
$$

Again, this is the mean over $\mathscr{C}_{j}$ of $u(x)=\sum_{j} u_{j+\frac{1}{2}} \mathbf{1}_{\mathscr{C}_{j+\frac{1}{2}}}(x)$. We obtain

$$
\begin{aligned}
& \frac{\bar{u}_{j}-u_{j}}{\Delta t}+\frac{G_{j+1}-G_{j}}{2 \delta x_{j+\frac{1}{2}}}+\frac{G_{j}-G_{j-1}}{2 \delta x_{j-\frac{1}{2}}}=0 \\
& \quad=\frac{\bar{u}_{j}-u_{j}}{\Delta t}+\frac{1}{\delta x_{j}}\left(\frac{\delta x_{j}}{2 \delta x_{j+\frac{1}{2}}} G_{j+1}+\left[\frac{\delta x_{j}}{2 \delta x_{j-\frac{1}{2}}}-\frac{\delta x_{j}}{2 \delta x_{j+\frac{1}{2}}}\right] G_{j}-\frac{\delta x_{j}}{2 \delta x_{j-\frac{1}{2}}} G_{j-1}\right) .
\end{aligned}
$$

The coefficient for $G_{j}$ can be recast as

$$
\frac{\delta x_{j+1}}{2 \delta x_{j+\frac{1}{2}}}-\underbrace{\frac{\delta x_{j}+\delta x_{j+1}}{2 \delta x_{j+\frac{1}{2}}}}_{=1}-\frac{\delta x_{j-1}}{2 \delta x_{j-\frac{1}{2}}}+\underbrace{\frac{\delta x_{j}+\delta x_{j-1}}{2 \delta x_{j-\frac{1}{2}}}}_{=1}=\frac{\delta x_{j+1}}{2 \delta x_{j+\frac{1}{2}}}-\frac{\delta x_{j-1}}{2 \delta x_{j-\frac{1}{2}}} .
$$

Therefore, we arrive at

$$
\frac{\bar{u}_{j}-u_{j}}{\Delta t}+\frac{G_{j+\frac{1}{2}}-G_{j-\frac{1}{2}}}{\delta x_{j}}=0
$$

with

$$
G_{j+\frac{1}{2}}=\frac{\delta x_{j} G_{j+1}+\delta x_{j+1} G_{j}}{2 \delta x_{j+\frac{1}{2}}}
$$


It can be interpreted as $G_{j+\frac{1}{2}}=\omega\left(x_{j+\frac{1}{2}}\right)$ with

$$
\omega(x)=\frac{x-x_{j}}{\delta x_{j+\frac{1}{2}}}\left(G_{j+1}-G_{j}\right)+G_{j}
$$

since $x_{j+\frac{1}{2}}-x_{j}=\delta x_{j} / 2$ and $2 \delta x_{j+\frac{1}{2}}=\delta x_{j}+\delta x_{j+1}$.

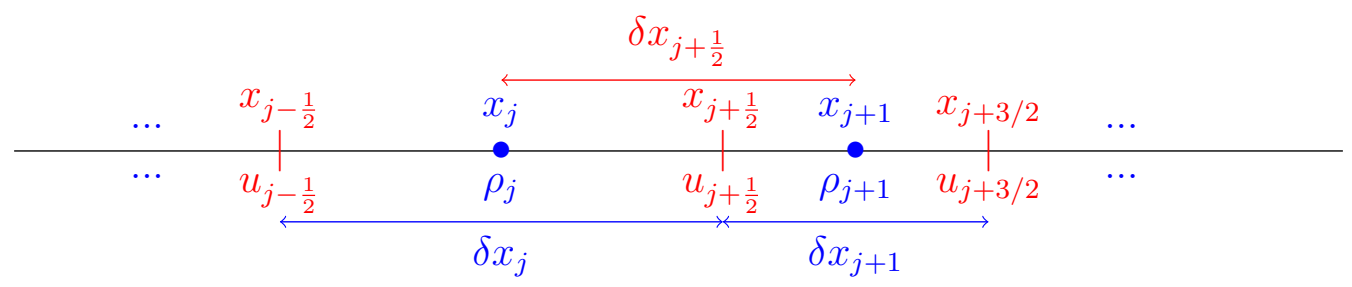

Figure 6: Staggered grid in dimension one.

We turn to the duality formula. We start by evaluating the discrete version of

$$
I=\frac{1}{\delta x_{j+\frac{1}{2}}} \int_{C_{j+\frac{1}{2}}}(\rho \operatorname{div} u+u \nabla \rho) \mathrm{d} x=\frac{\rho u\left(x_{j+1}\right)-\rho u\left(x_{j}\right)}{\delta x_{j+\frac{1}{2}}} .
$$

We already have at hand the product $u_{j+\frac{1}{2}}(\nabla \rho)_{j+\frac{1}{2}}=u_{j+\frac{1}{2}}\left(\rho_{j+1}-\rho_{j}\right) / \delta x_{j+\frac{1}{2}}$. The product $\rho \operatorname{div} u$ is not naturally evaluated on $x_{j+\frac{1}{2}}$ and we make use of the following formula

$$
\begin{aligned}
(\rho \operatorname{div} u)_{j+\frac{1}{2}} & =\frac{1}{2 \delta x_{j+\frac{1}{2}}}\left(\delta x_{j} \rho_{j}(\operatorname{div} u)_{j}+\delta x_{j+1} \rho_{j+1}(\operatorname{div} u)_{j+1}\right) \\
& =\frac{1}{2 \delta x_{j+\frac{1}{2}}}\left(\delta x_{j} \rho_{j} \frac{u_{j+\frac{1}{2}}-u_{j-\frac{1}{2}}}{\delta x_{j}}+\delta x_{j+1} \rho_{j+1} \frac{u_{j+\frac{3}{2}}-u_{j+\frac{1}{2}}}{\delta x_{j+1}}\right) .
\end{aligned}
$$

Summing these identities, we get

$$
\begin{aligned}
I_{j+\frac{1}{2}} & =u_{j+\frac{1}{2}}(\nabla \rho)_{j+\frac{1}{2}}+(\rho \operatorname{div} u)_{j+\frac{1}{2}} \\
& =u_{j+\frac{1}{2}} \frac{\rho_{j+1}-\rho_{j}}{\delta x_{j+\frac{1}{2}}}+\frac{1}{2 \delta x_{j+\frac{1}{2}}}\left(\rho_{j}\left(u_{j+\frac{1}{2}}-u_{j-\frac{1}{2}}\right)+\rho_{j+1}\left(u_{j+\frac{3}{2}}-u_{j+\frac{1}{2}}\right)\right) \\
& =\frac{1}{2 \delta x_{j+\frac{1}{2}}}\left(u_{j+\frac{1}{2}}\left(\rho_{j+1}-\rho_{j}\right)+\rho_{j+1} u_{j+\frac{3}{2}}-\rho_{j} u_{j-\frac{1}{2}}\right) \\
& =\frac{1}{2 \delta x_{j+\frac{1}{2}}}\left(\rho_{j+1}\left(u_{j+\frac{3}{2}}+u_{j+\frac{1}{2}}\right)-\rho_{j}\left(u_{j+\frac{1}{2}}+u_{j-\frac{1}{2}}\right)\right) \\
& =\frac{1}{\delta x_{j+\frac{1}{2}}}\left(\rho_{j+1} u_{j+1}-\rho_{j} u_{j}\right) .
\end{aligned}
$$

The other way around, we can also consider the duality relation on $\mathscr{C}_{j}$. Now, we have at hand the quantity $\rho_{j}(\operatorname{div} u)_{j}=\rho_{j}\left(u_{j+\frac{1}{2}}-u_{j-\frac{1}{2}}\right) / 2$ and we need to define the 
product $(u \nabla \rho)_{j}$. We set

$$
\begin{aligned}
(u \nabla \rho)_{j} & =\frac{1}{2}\left(u_{j+\frac{1}{2}} \nabla \rho_{j+\frac{1}{2}}+u_{j-\frac{1}{2}} \nabla \rho_{j-\frac{1}{2}}\right) \\
& =\frac{1}{2}\left(u_{j+\frac{1}{2}} \frac{\rho_{j+1}-\rho_{j}}{\delta x_{j+\frac{1}{2}}}+u_{j-\frac{1}{2}} \frac{\rho_{j}-\rho_{j-1}}{\delta x_{j-\frac{1}{2}}}\right) .
\end{aligned}
$$

Therefore, the quantity $\frac{1}{\delta x_{j}} \int_{\mathscr{C}_{j}}(\rho \operatorname{div} u+u \nabla \rho) \mathrm{d} x=\left(\rho u\left(x_{j+\frac{1}{2}}\right)-\rho u\left(x_{j-\frac{1}{2}}\right)\right) / \delta x_{j}$ is approximated by

$$
\begin{aligned}
I_{j}= & \rho_{j}(\operatorname{div} u)_{j}+(u \nabla \rho)_{j} \\
= & \frac{1}{\delta x_{j}}\left(u_{j+\frac{1}{2}}\left[\rho_{j}\left(1-\frac{\delta x_{j}}{2 \delta x_{j+\frac{1}{2}}}\right)+\frac{\delta x_{j}}{2 \delta x_{j+\frac{1}{2}}} \rho_{j+1}\right]\right. \\
& \left.\quad-u_{j-\frac{1}{2}}\left[\rho_{j}\left(\frac{\delta x_{j}}{2 \delta x_{j-\frac{1}{2}}}-1\right)-\frac{\delta x_{j}}{2 \delta x_{j-\frac{1}{2}}} \rho_{j-1}\right]\right) .
\end{aligned}
$$

Owing to the identity

$$
\frac{\delta x_{j+\frac{1}{2}}-\delta x_{j} / 2}{\delta x_{j+\frac{1}{2}}}=\frac{\delta x_{j+1} / 2+\delta x_{j} / 2-\delta x_{j} / 2}{\delta x_{j+\frac{1}{2}}}=\frac{\delta x_{j+1}}{2 \delta x_{j+\frac{1}{2}}},
$$

we arrive at

$$
I_{j}=\frac{1}{\delta x_{j}}\left(u_{j+\frac{1}{2}}\langle\rho\rangle_{j+\frac{1}{2}}-u_{j-\frac{1}{2}}\langle\rho\rangle_{j-\frac{1}{2}}\right)
$$

with

$$
\langle\rho\rangle_{j+\frac{1}{2}}=\frac{\delta x_{j+1} \rho_{j}+\delta x_{j} \rho_{j+1}}{2 \delta x_{j+\frac{1}{2}}}
$$

The weights in this formula correspond to the harmonic mean.

Hence, our goal is to extend these simple observations (which turns out to be useful for establishing consistency and the local conservation of the energy for schemes for the Euler equations on staggered grids $[19,23])$ to the two-dimensional framework, dealing with quite general and unstructured meshes.

\subsection{Transfer of conservative equations}

An important framework of application is the case where conservative fluxes $F_{K, \sigma}$ are considered. This is precisely the situation which arises for the simulation of conservation laws in fluid mechanics, with, for instance, the mass conservation equation, as explained in (Q1). In this specific case we can prove the following corollary to Theorem 2.7 .

Corollary 3.1. Let the hypotheses of Theorem 2.7 be fulfilled. Moreover, assume that the given fluxes $F_{K, \sigma}$ are conservative, which means that $F_{K, \sigma}=-F_{L, \sigma}$ if $\sigma=K \mid L \in$ 
$\mathscr{E}^{\mathfrak{T} \text {,int }}$ is the edge between $K$ and $L$. Then, there exist (flux-type) quantities $F_{D, \mathfrak{s}} \in \mathbb{R}$ for all $D \in \mathfrak{D}$, for all $\mathfrak{s} \in \mathscr{E}^{D}$ such that

$$
\begin{aligned}
& F_{D, \mathfrak{s}}=-F_{D^{\prime}, \mathfrak{s}} \text { if } \mathfrak{s}=D \mid D^{\prime} \in \mathscr{E}^{\mathfrak{D}, \text { int }} \text { is the edge between } D \text { and } D^{\prime} \text { (conservativity), } \\
& F_{D, \mathfrak{s}}=\sum_{K \in \mathfrak{T}} \sum_{\substack{\sigma^{D} \in \mathfrak{E}_{\mathfrak{\mathfrak { T }}, \text { ext }}^{\mathfrak{T}} \sigma^{D} \subset \mathfrak{F} \\
\sigma_{\mathfrak{O}}^{K}}} F_{K, \sigma^{D}}, \quad \text { if } \mathfrak{s} \in \mathscr{E}^{\mathfrak{D}, \text { ext }} \cap \mathscr{E}^{D},
\end{aligned}
$$

and

$$
|D| \operatorname{div}^{D}=\sum_{K \in \mathfrak{T}}|K \cap D| \operatorname{div}^{K}, \quad \forall D \in \mathfrak{D} .
$$

where

$$
\operatorname{div}^{D}=\frac{1}{|D|} \sum_{\mathfrak{s} \in \mathscr{E}^{D}} F_{D, \mathfrak{s}} \quad \text { and } \quad \operatorname{div}^{K}=\frac{1}{|K|} \sum_{\sigma \in \mathscr{E}^{K}} F_{K, \sigma} .
$$

Proof. Let $D \in \mathfrak{D}$. As already pointed out in Remark 2.8, we can apply Theorem 2.7 by setting

$$
F_{K, \sigma^{D}}=\frac{|\sigma \cap D|}{|\sigma|} F_{K, \sigma}, \quad \forall D \in \mathfrak{D}^{\sigma} .
$$

We obtain the following equality

$$
|D| \operatorname{div}^{D}=\sum_{K \in \mathfrak{T}}|K \cap D| \operatorname{div}^{K}-\sum_{K \in \mathfrak{T}} \sum_{\sigma \in \mathscr{E}^{K}} \frac{|\sigma \cap \stackrel{\circ}{ }|}{|\sigma|} F_{K, \sigma} .
$$

A reordering of the terms in the second sum of the right hand side leads to

$$
|D| \operatorname{div}^{D}=\sum_{K \in \mathfrak{T}}|K \cap D| \operatorname{div}^{K}-\sum_{\substack{\sigma \in \mathscr{E}_{\mathfrak{T}, \text {,int }} \\ \sigma=K \mid L}} \frac{|\sigma \cap \stackrel{\circ}{D}|}{|\sigma|}\left(F_{K, \sigma}+F_{L, \sigma}\right) .
$$

In the case of conservative fluxes, we have $F_{K, \sigma}+F_{L, \sigma}=0$ so that the conclusion is obtained.

This statement allows us to go back to the question (Q1) addressed in the Introduction. On the mesh $\mathfrak{T}$, we have the discrete conservation equation

$$
\frac{\bar{\rho}_{K}-\rho_{K}}{\Delta t}+\operatorname{div}^{K}=0,
$$

where

$$
\operatorname{div}^{K}=\frac{1}{|K|} \sum_{\sigma \in \mathscr{E}^{K}} F_{K, \sigma}
$$

involves the conservative numerical fluxes $F_{K, \sigma}$. We transfer the quantity $\rho$ to the twin grid $\mathfrak{D}$ by setting

$$
\rho_{D}=\sum_{\substack{K \in \mathfrak{T} \\ K \cap D \neq \emptyset}} \frac{|K \cap D|}{|D|} \rho_{K}
$$


Corollary 3.1 proves the existence of new numerical fluxes $F_{D, \mathfrak{s}}$ on $\partial D$ such that a discrete conservative equation

$$
\frac{\bar{\rho}_{D}-\rho_{D}}{\Delta t}+\operatorname{div}^{D}=0
$$

still holds, with $\operatorname{div}^{D}=\frac{1}{|D|} \sum_{s \in \mathscr{E} D} F_{D, \mathfrak{s}}$ and $F_{D, \mathfrak{s}}$ satisfying the conservation property

$$
F_{D, \mathfrak{s}}=-F_{D^{\prime}, \mathfrak{s}} \text { if } \mathfrak{s}=D \mid D^{\prime} \in \mathscr{E}^{\mathfrak{D}, \text { int }} .
$$

Indeed, we have

$$
\begin{array}{rlrl}
\frac{\bar{\rho}_{D}-\rho_{D}}{\Delta t} & =\sum_{\substack{K \in \mathfrak{T} \\
K \cap D \neq \emptyset}} \frac{|K \cap D|}{|D|} \frac{\bar{\rho}_{K}-\rho_{K}}{\Delta t} & \text { from the definition of } \rho_{D} \\
& =-\sum_{\substack{K \in \mathfrak{T} \\
K \cap D \neq \emptyset}} \frac{|K \cap D|}{|D|} \operatorname{div}^{K} & & \text { from the discrete mass balance on } \mathfrak{T} \\
& =-\operatorname{div}^{D} & & \text { from Corollary 3.1. }
\end{array}
$$

\subsection{Local discrete duality relations}

In this section, we show that Theorem 2.7 can also be useful to prove discrete duality relations between divergence and gradient operators defined on staggered grids. We have in mind finite volume discretizations of systems where the different discrete unknowns are not defined at the same location: roughly speaking we consider discretizations where the unknowns can be defined either at the cell centers or at the edges of a mesh $\mathfrak{T}$.

Therefore, in this discussion, the construction of the mesh $\mathfrak{D}$ depends on the geometry of the primal mesh $\mathfrak{T}$. In contrast to the results of the previous section, which were general, the two meshes $\mathfrak{T}$ and $\mathfrak{D}$ do not play a symmetric role anymore and we shall now need additional assumptions, related to this construction.

\subsubsection{Framework and application of Theorem 2.7}

To formulate the additional assumptions on the meshes $\mathfrak{D}$ and $\mathfrak{T}$, we distinguish the cells of $\mathfrak{D}$ the edges of which do not intersect the boundary of the domain $\Omega$ from the others. We set

$$
\mathfrak{D}^{\text {int }}=\left\{D \in \mathfrak{D} ; \mathscr{E}^{\mathfrak{D}, \text { ext }} \cap \mathscr{E}^{D}=\emptyset\right\} \quad \text { and } \quad \mathfrak{D}^{\text {ext }}=\mathfrak{D} \backslash \mathfrak{D}^{\text {int }} .
$$

We assume that the mesh $\mathfrak{T}$ splits a cell $D \in \mathfrak{D}^{\text {int }}$ in exactly two parts, so that we can associate the cell $D$ and the part of the edges that split it into two parts. A cell $D \in \mathfrak{D}^{\text {ext }}$ is assumed to be split in at most two parts, the non-split cells being possibly used to set boundary conditions. More precisely, we adopt the following hypothesis.

Assumption 3.2. We assume that there exists a partition of $\mathfrak{D}^{\text {ext }}$ in the form $\mathfrak{D}^{\text {ext }}=$ $\mathfrak{D}^{\text {ext, }, \partial} \cup \mathfrak{D}^{\text {ext, } \not \supset}$ such that 


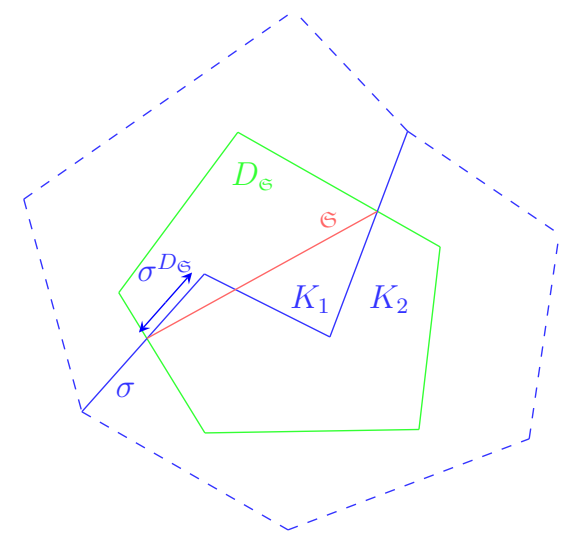

Figure 7: Fictitious edges.

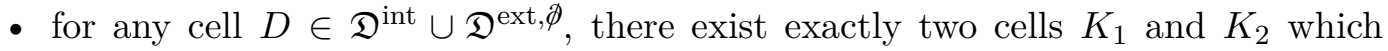
intersect the cell $D$; accordingly, the edges $\sigma^{D} \in \mathfrak{E}_{\mathfrak{D}}^{\mathfrak{T}}$ intersecting the interior of $D$ are interfaces of $K_{1}, K_{2}$ and we suppose they form a connected set;

- for any cell $D \in \mathfrak{D}^{\text {ext, } \partial}$, there exists a cell $K_{1} \in \mathfrak{T}$ such that $D \subset K_{1}$.

Remark 3.3. Assumption 3.2 induces geometrical restrictions. It covers many relevant situations, but it excludes some cases like the covolume/FVE meshes and the MAC schemes, where each diamond intersects three or four cells of $\mathfrak{T}$. It is likely that the present framework can be adapted to handle these specific schemes, for instance inspired from [1] for the covolume/FVE meshes, or by suitably combining formula of the one-dimensional framework, see Section 3.1 and [23].

Thus, the interior of a cell $D \in \mathfrak{D}^{\text {int }} \cup \mathfrak{D}^{\text {ext }, \not}$ may be crossed by several consecutive edges $\sigma^{D} \in \mathfrak{E}_{\mathfrak{D}}^{\mathfrak{T}}$ but all these edges must share the same elements $K_{1}$ and $K_{2}$. To handle all these edges at the same time, it is possible and useful to introduce a fictitious edge, denoted $\mathfrak{s}$, which is defined as the segment joining the starting and ending points of the sequence of the consecutive edges in $\mathfrak{E}_{\mathfrak{D}}^{\mathfrak{T}}$ crossing $D$ (see Fig. 7). There exists a one-to-one application between the fictitious edges $\mathfrak{S}$ and the cells $D$ of $\mathfrak{D}^{\text {int }} \cup \mathfrak{D}^{\text {ext, } \not \supset}$. To bring forward this connection we use the notation $D_{\mathfrak{S}}$ and, with a slight abuse of notation, $\mathfrak{S}=K_{1} \mid K_{2}$ means that $K_{1}$ and $K_{2}$ are the two cells that intersect the interior of $D_{\mathfrak{S}}$. We denote by $\mathbf{n}_{K_{1}, \mathfrak{S}}$ (resp. $\mathbf{n}_{K_{2}, \mathfrak{S}}$ ) the unit normal vector to $\mathfrak{S}$ from $K_{1}$ to $K_{2}$ (resp. from $K_{2}$ to $K_{1}$ ). Note that we have

$$
\mathbf{n}_{K_{1}, \mathfrak{S}}=-\mathbf{n}_{K_{2}, \mathfrak{S}}
$$

and, using Lemma 2.1,

$$
|\mathfrak{S}| \mathbf{n}_{K_{i}, \mathfrak{S}}=\sum_{\substack{\sigma^{D} \in \mathfrak{E}_{\mathfrak{D}}^{K_{i}} \\ \sigma^{D} \subset D_{\mathfrak{S}}, \sigma^{D} \not \subset \partial D_{\mathfrak{S}}}}\left|\sigma^{D}\right| \mathbf{n}_{K_{i}, \sigma^{D}}, \quad i=1,2 .
$$




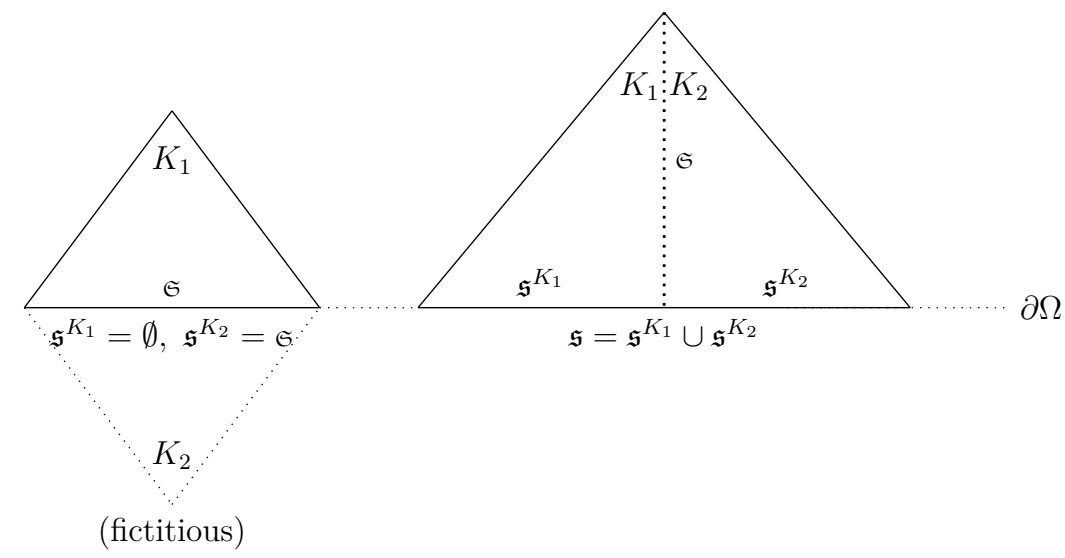

Figure 8: Two types of cells at the boundary.

By convention, we denote by $F_{K_{i}, \mathfrak{S}}$ the following flux

$$
F_{K_{i}, \mathfrak{S}}=\sum_{\substack{\sigma^{D} \in \mathfrak{E}_{\mathfrak{O}}^{K_{i}} \\ \sigma^{D} \subset D_{\mathfrak{S}}, \sigma^{D} \not \subset \partial D_{\mathfrak{S}}}} F_{K_{i}, \sigma^{D}}, \quad i=1,2 .
$$

For $D \in \mathfrak{D}^{\text {ext }}$, it is also useful to introduce fictitious edges to gather in a single notation the boundary edges. We refer the reader to Fig. 8 for following the forthcoming discussion.

For $D_{\mathfrak{S}} \in \mathfrak{D}^{\text {ext }, \not}, \mathfrak{S}=K_{1} \mid K_{2}$, we use the notation $\mathfrak{S}^{\partial, K_{i}}, i=1,2$ to handle the part of boundary edges of $K_{i}$ embedded in $D_{\mathfrak{E}}$, that is the edges $\sigma^{D} \in \mathfrak{E}_{\mathfrak{O}}^{\mathfrak{P} \text {,ext }} \cap \mathfrak{E}_{\mathfrak{O}}^{K_{i}}$ such that $\sigma^{D} \subset D_{\mathfrak{S}}$. The fictitious boundary edges $\mathfrak{S}^{\partial, K_{i}}$ are defined as the segments joining the starting and ending points of these consecutive edges. Then, we set (see Lemma 2.1)

$$
\left|\mathfrak{S}^{\partial, K_{i}}\right| \mathbf{n}_{K_{i}, \mathfrak{S}^{\partial, K_{i}}}=\sum_{\substack{\sigma^{D} \in \mathfrak{E}_{\mathfrak{\mathcal { T }}, \text { ext }}^{\text {a }} \cap \mathfrak{E}_{\mathcal{O}}^{K_{i}} \\ \sigma^{D} \subset D_{\mathfrak{S}}}}\left|\sigma^{D}\right| \mathbf{n}_{K_{i}, \sigma^{D}}, \quad i=1,2 .
$$

and

$$
F_{K_{i}, \mathfrak{S}^{\partial, K_{i}}}=\sum_{\substack{\sigma^{D} \in \mathfrak{E}_{\mathfrak{T}}^{\mathfrak{T}, \text { ext }} \cap \mathfrak{E}_{\mathfrak{O}}^{\sigma^{D}} \\ \sigma^{K_{i}} \subset D_{\mathfrak{S}}}} F_{K_{i}, \sigma^{D}}, \quad i=1,2 .
$$

For $D \in \mathfrak{D}^{\text {ext }, \partial}$, by assumption, there exists a cell $K_{1} \in \mathfrak{T}$ such that $D \subset K_{1}$. For the sake of uniformity of notation, we denote again $D=D_{\mathfrak{S}}$ with $\mathfrak{S}=K_{1} \mid K_{2}$. In this case, $K_{2}$ may be considered as a fictitious cell exterior to the domain. Associating an unknown to the fictitious cell $K_{2}$ can be useful to take into account boundary conditions. The notation $\mathfrak{S}$ allows us to handle the boundary edges of $K_{1}$ embedded in $D_{\mathfrak{S}}$, that is the edges $\sigma^{D} \in \mathfrak{E}_{\mathfrak{D}}^{\mathfrak{D} \text {,ext }} \cap \mathfrak{E}_{\mathfrak{Q}}^{K_{1}}$ such that $\sigma^{D} \subset D_{\mathfrak{S}}$. Thus, $\mathfrak{S}$ is defined here as the fictitious boundary edge joining the starting and ending points of these consecutive 
edges. Then we set

$$
|\mathfrak{S}| \mathbf{n}_{K_{1}, \mathfrak{S}}=\sum_{\substack{\sigma^{D} \in \mathfrak{E}_{\mathfrak{T}, \text { ext }}^{\mathfrak{D}} \cap \mathfrak{E}_{\mathfrak{D}}^{K_{1}} \\ \sigma^{D} \subset D_{\mathfrak{S}}}}\left|\sigma^{D}\right| \mathbf{n}_{K_{1}, \sigma^{D}}
$$

and

$$
F_{K_{1}, \mathfrak{S}}=\sum_{\substack{\sigma^{D} \in \mathfrak{E}_{\mathfrak{Q}}^{\mathfrak{T}, \text { ext }}, \mathfrak{E}_{\mathfrak{P}}^{K_{1}} \\ \sigma^{D} \subset D_{\mathfrak{S}}}} F_{K_{1}, \sigma^{D}}
$$

This provides a way to describe all the edges of a cell $K \in \mathfrak{T}$ gathering the ones which belong to the same cell of $\mathfrak{D}$. Indeed, if we introduce the following sets

$$
\begin{aligned}
& \mathfrak{D}^{K}=\left\{D_{\mathfrak{S}} \in \mathfrak{D} ; D_{\mathfrak{S}} \cap K \neq \emptyset\right\}, \\
& \mathfrak{D}^{K, \not \supset}=\mathfrak{D}^{K} \cap \mathfrak{D}^{\mathrm{ext}, \not},
\end{aligned}
$$

we obtain a description of $\operatorname{div}^{K}$ as follows

$$
\operatorname{div}^{K}=\frac{1}{|K|}\left(\sum_{D_{\mathfrak{S}} \in \mathfrak{D}^{K}} F_{K, \mathfrak{S}}+\sum_{D_{\mathfrak{S}} \in \mathfrak{D}^{K, \not}} F_{K, \mathfrak{S}}{ }^{\partial, K}\right) .
$$

In the right hand side the boundary fluxes are distributed among the two sums: the first sum contains interior fluxes but also the boundary fluxes through the edges of the cells $D_{\mathfrak{S}}$ that are split into two parts; the second sum only contains the boundary fluxes through the edges of the cells $D_{\mathfrak{S}}$ that are not split.

An edge $\mathfrak{s} \in \mathscr{E}^{\mathfrak{D}}$ of a cell $D_{\mathfrak{S}} \in \mathfrak{D}$ can be split into (at most) two parts by the mesh $\mathfrak{T}$. We introduce the following notation (see Fig. 8)

- for all $D_{\mathfrak{S}} \in \mathfrak{D}^{\text {int }} \cup \mathfrak{D}^{\text {ext, } \not \supset}, \mathfrak{S}=K_{1} \mid K_{2}$,

$$
\mathfrak{s}^{K_{i}}=\mathfrak{s} \cap K_{i}, \quad i=1,2, \quad \forall \mathfrak{s} \in \mathscr{E}^{D_{\mathfrak{S}}} .
$$

- for all $D_{\mathfrak{S}} \in \mathfrak{D}^{\text {ext, } \partial}, \mathfrak{S}=K_{1} \mid K_{2}$,

$$
\begin{array}{ll}
\mathfrak{s}^{K_{1}}=\mathfrak{s}, \mathfrak{s}^{K_{2}}=\emptyset, & \forall \mathfrak{s} \in \mathscr{E}^{D_{\mathfrak{S}}} \cap \mathscr{E}^{\mathfrak{D}, \text { int }}, \\
\mathfrak{s}^{K_{1}}=\emptyset, \mathfrak{s}^{K_{2}}=\mathfrak{s}, \quad \forall \mathfrak{s} \in \mathscr{E}^{D_{\mathfrak{S}} \cap \mathscr{E}^{\mathcal{D}, \text { ext }}}
\end{array}
$$

Thus, when the cell $D_{\mathfrak{S}}$ is split into two parts, $\mathfrak{s}^{K_{1}}$ and $\mathfrak{s}^{K_{2}}$ stand for the part of $\mathfrak{s}$ (possibly empty) embedded respectively in $K_{1}$ and $K_{2}$. If the cell $D_{\mathfrak{S}}$ is fully embedded in $K_{1}$, we adopt the convention that all interior edges are associated to $K_{1}$ whereas the boundary edges are associated to $K_{2}$.

We can now translate the statement of Thereom 2.7 in the framework presented above.

Corollary 3.4. Let the assumptions of Theorem 2.7 be fulfilled, so that (flux-type) quantities $F_{K, \sigma^{D}} \in \mathbb{R}$ are given for all $K \in \mathfrak{T}$, for all $\sigma^{D} \in \mathfrak{E}_{\mathfrak{D}}^{K}$. We assume moreover 
that Assumption 3.2 holds. Then, there exist (flux-type) quantities $F_{D_{\mathfrak{S}, \mathfrak{s}}} \in \mathbb{R}$ for all $D_{\mathfrak{S}} \in \mathfrak{D}$, for all $\mathfrak{s} \in \mathscr{E}^{D_{\mathfrak{S}}}$ such that

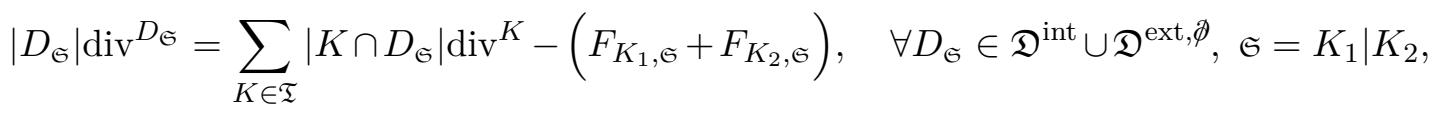

and

$$
\left|D_{\mathfrak{S}}\right| \operatorname{div}^{D_{\mathfrak{S}}}=\sum_{K \in \mathfrak{T}}\left|K \cap D_{\mathfrak{S}}\right| \operatorname{div}^{K}, \quad \forall D_{\mathfrak{S}} \in \mathfrak{D}^{\text {ext, }, \partial}
$$

where $\left|D_{\mathfrak{S}}\right| \operatorname{div}^{D_{\mathfrak{S}}}=\sum_{\mathfrak{s} \in \mathscr{E}^{D_{\mathfrak{S}}}} F_{D_{\mathfrak{S}, \mathfrak{s}}}$ and $\operatorname{div}^{K}$ is given by (16). Moreover the fluxes on the boundary of the domain satisfy

- for all $D_{\mathfrak{S}} \in \mathfrak{D}^{\mathrm{ext}, \not \supset}, \mathfrak{S}=K_{1} \mid K_{2}$,

$$
\sum_{\mathfrak{s} \in \mathscr{E}^{D_{\mathfrak{S} \cap \mathscr{E} \mathfrak{D}, \text { ext }}}} F_{D_{\mathfrak{S}, \mathfrak{s}}}=F_{K_{1}, \mathfrak{S}^{\partial, K_{1}}}+F_{K_{2}, \mathfrak{S}^{\partial, K_{2}}}
$$

- for all $D_{\mathfrak{S}} \in \mathfrak{D}^{\mathrm{ext}, \partial}, \mathfrak{S}=K_{1} \mid K_{2}$,

$$
\sum_{\mathfrak{s} \in \mathscr{E}^{D_{\mathfrak{S} \cap \mathscr{E} \mathfrak{D}, \text { ext }}}} F_{D_{\mathfrak{S}, \mathfrak{s}}}=F_{K_{1}, \mathfrak{G}}
$$

Proof. Let us first consider $D_{\mathfrak{S}} \in \mathfrak{D}^{\text {int }} \cup \mathfrak{D}^{\text {ext, } \not \supset}, \mathfrak{S}=K_{1} \mid K_{2}$. Theorem 2.7 leads to

$$
\left|D_{\mathfrak{S}}\right| \operatorname{div}^{D_{\mathfrak{S}}}=\sum_{K \in \mathfrak{T}}\left|K \cap D_{\mathfrak{S}}\right| \operatorname{div}^{K}-\sum_{K \in \mathfrak{T}} \sum_{\substack{\sigma^{D} \in \mathfrak{E}_{\mathfrak{D}}^{K} \\ \sigma^{D} \subset D_{\mathfrak{S}}, \sigma^{D} \not \subset \partial D_{\mathfrak{S}}}} F_{K, \sigma^{D}} .
$$

Assumption 3.2 ensures that all edges $\sigma^{D}$ appearing in the second sum of the right hand side share the elements $K_{1}$ and $K_{2}$ so that the sum over $K \in \mathfrak{T}$ reduces to a sum over $K_{1}$ and $K_{2}$. Moreover, by definition, we have

$$
F_{K_{i}, \mathfrak{S}}=\sum_{\substack{\sigma^{D} \in \mathfrak{F}_{\mathfrak{D}}^{K} \\ \sigma^{D} \subset D_{\mathfrak{S}}, \sigma^{D} \not \subset \partial D_{\mathfrak{S}}}} F_{K_{i}, \sigma^{D}}, \quad i=1,2 .
$$

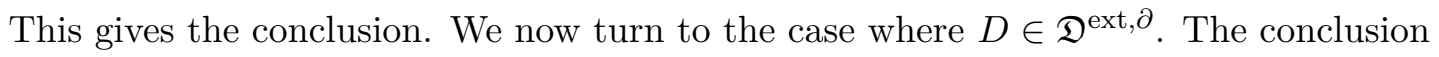
follows directly from Theorem 2.7 since there exists $K_{1} \in \mathfrak{T}$ such that $D \subset K_{1}$ and, consequently, there is no $\sigma^{D} \in \mathfrak{E}_{\mathfrak{D}}^{K_{1}}$ such that $\sigma^{D} \subset D$ and $\sigma^{D} \not \subset \partial D$.

Concerning boundary fluxes, for all $D_{\mathfrak{S}} \in \mathfrak{D}^{\text {ext }}$, Theorem 2.7 gives the expression

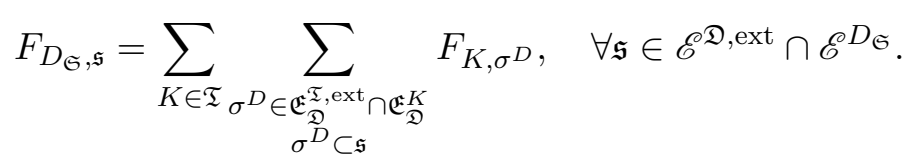

Hence, we obtain

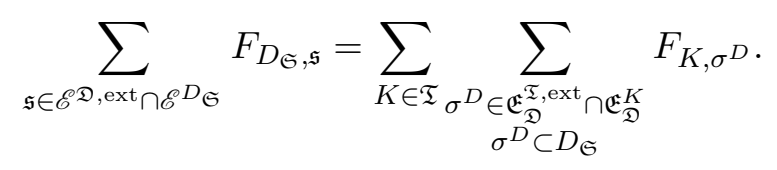


Now, if $D_{\mathfrak{S}} \in \mathfrak{D}^{\text {ext, } \not}, \mathfrak{S}=K_{1} \mid K_{2}$, by assumption, as previously, all edges $\sigma^{D}$ appearing in the sum of the right hand side share the elements $K_{1}$ and $K_{2}$ so that the sum over $K \in \mathfrak{T}$ reduces to a sum over $K_{1}$ and $K_{2}$ and the result is obtained by definition of $F_{K_{i}, \mathfrak{S}^{\partial, K_{i}}}, i=1,2$. If $D_{\mathfrak{S}} \in \mathfrak{D}^{\text {ext, }, \partial}, \mathfrak{S}=K_{1} \mid K_{2}$, the sum over $K \in \mathfrak{T}$ reduces to a sum over $K_{1}$ since $D_{\mathfrak{S}} \subset K_{1}$ and the conclusion is obtained by definition of $F_{K_{1}, \mathfrak{S}}$.

\subsubsection{Discrete operators}

We now consider a vector field $\mathbf{u}^{\mathfrak{T}}=\left(\mathbf{u}_{K}\right)_{K \in \mathfrak{T}}$ and a scalar field $\Psi^{\mathfrak{T}}=\left(\Psi_{K}\right)_{K \in \mathfrak{T}}$ located at the cell centers of $\mathfrak{T}$. We then define the following discrete operators at cell centers of $\mathfrak{D}$ : for all $D_{\mathfrak{S}} \in \mathfrak{D}, \mathfrak{S}=K_{1} \mid K_{2}$,

$$
\begin{aligned}
\operatorname{div}^{D_{\mathfrak{S}}} \mathbf{u}^{\mathfrak{T}}=\frac{1}{\left|D_{\mathfrak{S}}\right|} \sum_{\mathfrak{s} \in \mathscr{E} D_{\mathfrak{S}}} F_{D_{\mathfrak{S}, \mathfrak{F}}} & \text { with } F_{D_{\mathfrak{S}, \mathfrak{s}}}=\left(\left|\mathfrak{s}^{K_{1}}\right| \mathbf{u}_{K_{1}}+\left|\mathfrak{s}^{K_{2}}\right| \mathbf{u}_{K_{2}}\right) \cdot \mathbf{n}_{D_{\mathfrak{S}, \mathfrak{F}}}, \\
\nabla^{D_{\mathfrak{S}}} \Psi^{\mathfrak{T}}=\frac{1}{\left|D_{\mathfrak{S}}\right|} \sum_{\mathfrak{s} \in \mathscr{E}^{D} D_{\mathfrak{S}}} \mathbf{G}_{D_{\mathfrak{S}, \mathfrak{s}}} & \text { with } \mathbf{G}_{D_{\mathfrak{S}, \mathfrak{s}}}=\left(\left|\mathfrak{s}^{K_{1}}\right| \Psi_{K_{1}}+\left|\mathfrak{s}^{K_{2}}\right| \Psi_{K_{2}}\right) \mathbf{n}_{D_{\mathfrak{S}, \mathfrak{s}}}
\end{aligned}
$$

Note that, when $D_{\mathfrak{S}} \in \mathfrak{D}^{\text {ext, } \partial}, K_{2}$ is a fictitious cell, so that the values $\mathbf{u}_{K_{2}}$ or $\Psi_{K_{2}}$ have to be considered as data which allows us to impose boundary conditions. The operators $\operatorname{div}^{D_{\mathfrak{S}}}$ and $\nabla^{D_{\mathfrak{S}}}$ can be rewritten as explained in the following lemma, where we make a more natural expression appear, by means of finite differences. Note however, as it will be further discussed below, that the consistency of these discrete operators is not guaranteed; it requires additional assumption on the mesh geometry.

Lemma 3.5. The following equalities hold

$$
\left|D_{\mathfrak{S}}\right| \operatorname{div}^{D_{\mathfrak{S}}} \mathbf{u}^{\mathfrak{T}}=|\mathfrak{S}|\left(\mathbf{u}_{K_{2}}-\mathbf{u}_{K_{1}}\right) \cdot \mathbf{n}_{K_{1}, \mathfrak{S}}, \quad \forall D_{\mathfrak{S}} \in \mathfrak{D}, \mathfrak{S}=K_{1} \mid K_{2},
$$

and

$$
\left|D_{\mathfrak{S}}\right| \nabla^{D_{\mathfrak{S}}} \Psi^{\mathfrak{T}}=|\mathfrak{S}|\left(\Psi_{K_{2}}-\Psi_{K_{1}}\right) \mathbf{n}_{K_{1}, \mathfrak{S}}, \quad \forall D_{\mathfrak{S}} \in \mathfrak{D}, \mathfrak{S}=K_{1} \mid K_{2} .
$$

Proof. Let us consider $D_{\mathfrak{S}} \in \mathfrak{D}, \mathfrak{S}=K_{1} \mid K_{2}$. The proof relies on the following equalities:

$$
\sum_{\mathfrak{s} \in \mathscr{E} D}\left|\mathfrak{s}^{K_{1}}\right| \mathbf{n}_{D_{\mathfrak{S}, \mathfrak{s}}}=-|\mathfrak{S}| \mathbf{n}_{K_{1}, \mathfrak{S}} \quad \text { and } \quad \sum_{\mathfrak{s} \in \mathscr{E}^{D} \mathfrak{S}}\left|\mathfrak{s}^{K_{2}}\right| \mathbf{n}_{D_{\mathfrak{S}, \mathfrak{s}}}=-|\mathfrak{S}| \mathbf{n}_{K_{2}, \mathfrak{S}}
$$

If $D_{\mathfrak{S}} \in \mathfrak{D}^{\text {int }} \cup \mathfrak{D}^{\text {ext, } \not \nexists}, \mathfrak{S}=K_{1} \mid K_{2}$, the cell $D_{\mathfrak{S}}$ is split in two non-empty polygons $D_{\mathfrak{S}} \cap K_{1}$ and $D_{\mathfrak{S}} \cap K_{2}$. The equalities (18) come from Lemma 2.1 applied to these two polygons. If $D_{\mathfrak{S}} \in \mathfrak{D}^{\text {ext, }, \partial}, \mathfrak{S}=K_{1} \mid K_{2}$, the first equality is obtained by applying the Green formula on $D_{\mathfrak{S}}$ whereas, in the second equality, there is only one term in the sum of the left hand side which coincides exactly with the right hand side by definition.

Using these equalities leads to

$$
\begin{aligned}
\left|D_{\mathfrak{S}}\right| \operatorname{div}^{D_{\mathfrak{S}}} \mathbf{u}^{\mathfrak{T}} & =\sum_{\mathfrak{s} \in \mathscr{E} D_{\mathfrak{S}}}\left|\mathfrak{s}^{K_{1}}\right| \mathbf{u}_{K_{1}} \cdot \mathbf{n}_{D_{\mathfrak{S}, \mathfrak{s}}}+\sum_{\mathfrak{s} \in \mathscr{E}^{D_{\mathfrak{S}}}}\left|\mathfrak{s}^{K_{2}}\right| \mathbf{u}_{K_{2}} \cdot \mathbf{n}_{D_{\mathfrak{S}, \mathfrak{s}}} \\
& =\mathbf{u}_{K_{1}} \cdot \sum_{\mathfrak{s} \in \mathscr{E}^{D_{\mathfrak{S}}}}\left|\mathfrak{s}^{K_{1}}\right| \mathbf{n}_{D_{\mathfrak{S}, \mathfrak{s}}}+\mathbf{u}_{K_{2}} \cdot \sum_{\mathfrak{s} \in \mathscr{E}^{D_{\mathfrak{S}}}}\left|\mathfrak{s}^{K_{2}}\right| \mathbf{n}_{D_{\mathfrak{S}, \mathfrak{s}}}
\end{aligned}
$$


This relation combined with the equalities (18) leads to the conclusion. In the same vein, we have

$$
\begin{aligned}
\left|D_{\mathfrak{S}}\right| \nabla^{D_{\mathfrak{S}}} \Psi^{\mathfrak{T}} & =\sum_{\mathfrak{s} \in \mathscr{E} D_{\mathfrak{S}}}\left|\mathfrak{s}^{K_{1}}\right| \Psi_{K_{1}} \mathbf{n}_{D_{\mathfrak{S}, \mathfrak{s}}}+\sum_{\mathfrak{s} \in \mathscr{E} D_{\mathfrak{S}}}\left|\mathfrak{s}^{K_{2}}\right| \Psi_{K_{2}} \mathbf{n}_{D_{\mathfrak{S}, \mathfrak{F}}} \\
& =\Psi_{K_{1}} \sum_{\mathfrak{s} \in \mathscr{E}^{D_{\mathfrak{S}}}}\left|\mathfrak{s}^{K_{1}}\right| \mathbf{n}_{D_{\mathfrak{S}, \mathfrak{s}}}+\Psi_{K_{2}} \sum_{\mathfrak{s} \in \mathscr{E} D_{\mathfrak{S}}}\left|\mathfrak{s}^{K_{2}}\right| \mathbf{n}_{D_{\mathfrak{S}, \mathfrak{s}}}
\end{aligned}
$$

As previously, this relation combined with equalities (18) allows us to conclude.

The other way around, we consider a vector field $\mathbf{v}^{\mathfrak{D}}=\left(\mathbf{v}_{D_{\mathfrak{S}}}\right)_{D_{\mathfrak{S}} \in \mathfrak{D}}$ and a scalar field $\Phi^{\mathfrak{D}}=\left(\Phi_{D_{\mathfrak{S}}}\right)_{D_{\mathfrak{S}} \in \mathfrak{D}}$ now located at the cell centers of $\mathfrak{D}$. We then define the following discrete operators at cell centers of $\mathfrak{T}$ : for all $K \in \mathfrak{T}$,

$$
\begin{aligned}
\operatorname{div}^{K} \mathbf{v}^{\mathfrak{D}} & =\frac{1}{|K|}\left(\sum_{D_{\mathfrak{S}} \in \mathfrak{D}^{K}} G_{K, \mathfrak{S}}+\sum_{D_{\mathfrak{S}} \in \mathfrak{D}^{K, \nexists}} G_{K, \mathfrak{S}^{\partial, K}}\right) \\
\nabla^{K} \Phi^{\mathfrak{D}} & =\frac{1}{|K|}\left(\sum_{D_{\mathfrak{S}} \in \mathfrak{D}^{K}} \mathbf{F}_{K, \mathfrak{S}}+\sum_{D_{\mathfrak{S}} \in \mathfrak{D}^{K, \not}} \mathbf{F}_{K, \mathfrak{S}^{\partial, K}}\right)
\end{aligned}
$$

with

$$
\begin{aligned}
G_{K, \mathfrak{S}}=|\mathfrak{S}| \mathbf{v}_{D_{\mathfrak{S}}} \cdot \mathbf{n}_{K, \mathfrak{S}} \quad \text { and } \quad \mathbf{F}_{K, \mathfrak{S}}=|\mathfrak{S}| \Phi_{D_{\mathfrak{S}}} \mathbf{n}_{K, \mathfrak{S}}, \\
G_{K, \mathfrak{S}, K}=\left|\mathfrak{S}^{\partial, K}\right| \mathbf{v}_{D_{\mathfrak{S}}} \cdot \mathbf{n}_{K, \mathfrak{S}}{ }^{\partial, K} \quad \text { and } \quad \mathbf{F}_{K, \mathfrak{S}}{ }^{\partial, K}=\left|\mathfrak{S}^{\partial, K}\right| \Phi_{D_{\mathfrak{S}}} \mathbf{n}_{K, \mathfrak{S}}{ }^{\partial, K} .
\end{aligned}
$$

As said above, the operators defined in (17) (and probably (19)) are not always consistent, as it is clear from the formula in Lemma 3.5. The consistency requires additional assumptions on the mesh $\mathfrak{T}$, like orthogonality conditions, or it requires to combine different operators defined on several meshes. Note that nevertheless a convergence of these operators may hold in a weak sense, see [15].

We are going to investigate in further details the cases of the Two-Point flux approximation scheme or the discrete duality finite volume discretization.

Two-Point flux approximation (TPFA) Assume that we have at hand a mesh $\mathfrak{T}$, admissible in the sense of [15, Definition 3.1]. In particular, given two control volumes $\overline{K \text { and } L}$ in $\mathfrak{T}$, with common edge $\sigma$, and "representative" points $x_{K} \in K$, $x_{L} \in L$, at which the information is stored (for instance the circumcenter of the cells for triangular meshes) the axis $x_{K} x_{L}$ is orthogonal to $\sigma$. We denote $\mathfrak{D}$ the diamond mesh associated to $\mathfrak{T}$ which is obtained by joining the vertices of $\mathfrak{T}$ to the $x_{K}$ 's, see Fig. 9. In this case, an interior cell of $\mathfrak{D}$ is exactly split into two cells by a single and whole edge of $\mathscr{E}^{\mathfrak{T}}$ and a boundary cell of $\mathfrak{D}$ is always fully embedded in a cell of $\mathfrak{T}$, that is $\mathfrak{D}^{\text {ext, } \not \supset}=\emptyset$. There is a one to one application between the cells of $\mathfrak{D}$ and the edges $\sigma$ of $\mathfrak{T}$. Thus, the notation $\mathfrak{S}$ may be understood as a single whole edge $\sigma$ and the cells of $\mathfrak{D}$ can be denoted $D_{\sigma}$. The operator $\nabla^{D_{\mathfrak{S}}}$ and $\operatorname{div}^{K}$ defined in (17) and (19) are exactly the discrete gradient and divergence of the so-called TPFA scheme, see e. g. [11, Section 2]. 


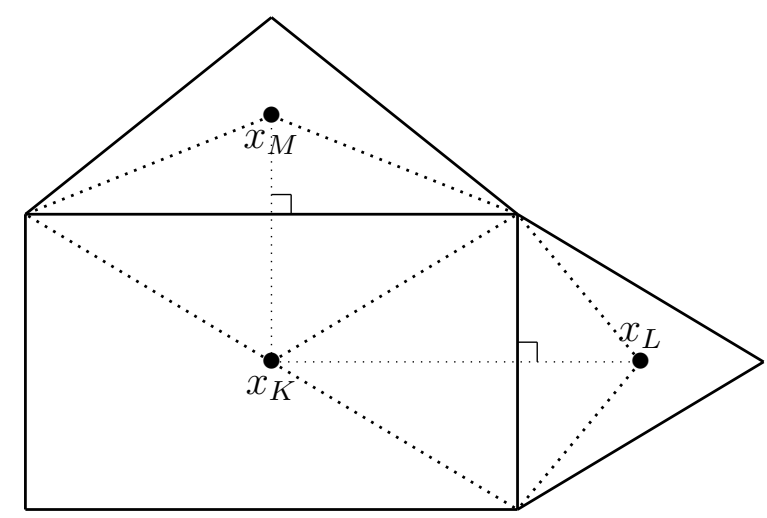

Figure 9: TPFA meshes

Discrete duality finite volume discretization (DDFV) Consider now that we have at hand a primal mesh $\mathfrak{T}$, a dual mesh $\mathfrak{T}^{*}$ and a diamond mesh $\mathfrak{D}$, see Fig. 10. We use the DDFV framework, for which we refer the reader to the seminal papers $[2,7,10,21,22]$.
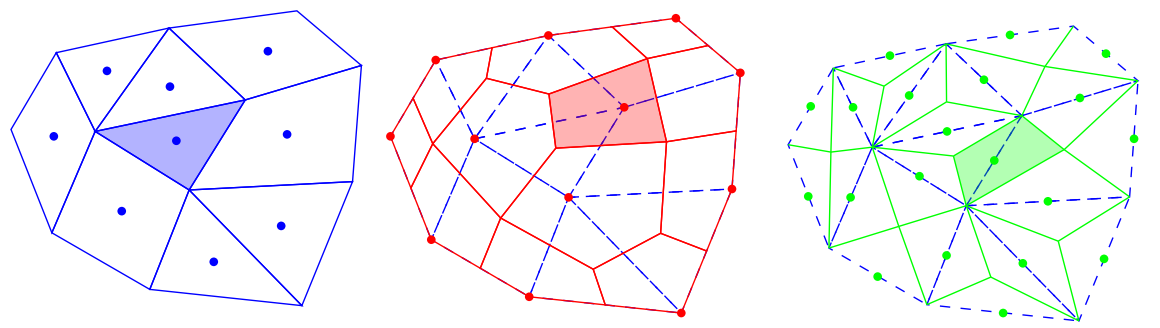

Figure 10: Primal, dual and diamond meshes in the DDFV scheme.

The construction of the meshes proceeds as follows:

- The primal mesh $\mathfrak{T}$ is made of disjoint, non-degenerate, convex polygons $K$ called "primal cells". We associate to each cell $K$, a point $x_{K} \in K$ (see the blue cell in Fig. 10).

- The diamond mesh $\mathfrak{D}$ is made of quadrilateral cells obtained by joining the endpoints of the edges $\sigma$ of the primal mesh to the centers $x_{K}$ and $x_{L}$ of the primal cells that share this edge (see Fig. 10). Thus, the edge $\sigma$ and the segment $\left[x_{K}, x_{L}\right]$ are the two diagonals of the diamond cells. Note that boundary diamond cells (that is the case where $\sigma \subset \partial \Omega$ ) degenerate to triangles, see Fig. 10).

- The dual mesh $\mathfrak{T}^{*}$ is made of cells built around the vertices $x_{K^{*}}$ of the primal mesh (see the red cell in Fig. 10). In the sequel, we consider the following two options, depicted in Fig. 11:

- with the direct approach the interior dual mesh consists of cells $K^{*}$, built around the vertex $x_{K^{*}} \notin \partial \Omega$, by joining the centers $x_{K}$ of all cells having 


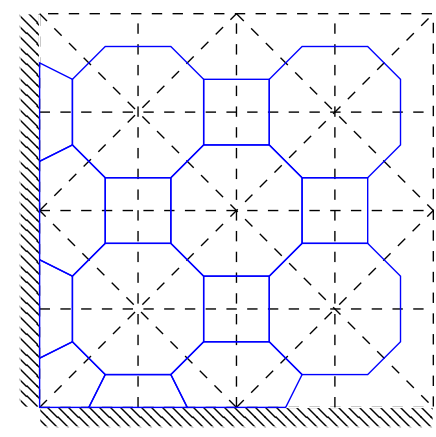

(a) Direct dual mesh

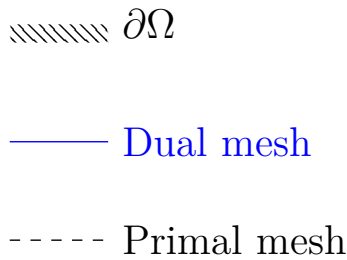

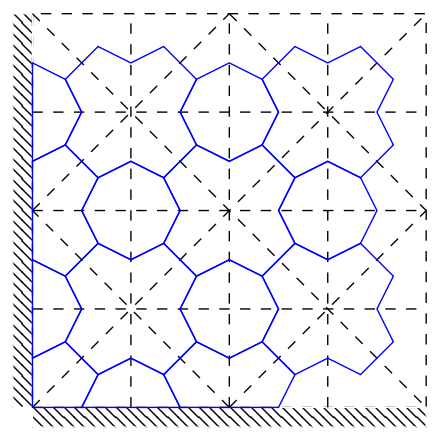

(b) Barycentric dual mesh

Figure 11: Structured triangular mesh

$K^{*}$ as a vertex (see the red cell in Fig. 10 or Fig. 11 at left). The boundary dual mesh is the set of cells $K^{*}$ such that $x_{K^{*}} \in \partial \Omega$ and in this specific case, a dual cell is made by joining the centers of the cells that share the vertex $x_{K^{*}}$ and the centers of the two boundary edges containing $x_{K^{*}}$.

- the barycentric mesh is obtained by joining the centers $x_{K}$ to the midpoints of the edges of the primal mesh [8,9] (see Fig. 11 at right).

The three meshes cover the computational domain:

$$
\Omega=\bigcup_{K \in \mathfrak{T}} K=\bigcup_{K^{*} \in \mathfrak{T}^{*}} K^{*}=\bigcup_{D \in \mathfrak{D}} D
$$

The barycentric dual mesh might have a very complex structure, but in certain circumstances it leads to a better numerical performance. For the analysis of DDFV schemes, it turns out that working with the direct mesh induces geometric constraints. Hence, we distinguish the following two situations:

- either all diamond cells are convex and we work with the direct dual mesh. In such a case the diagonals $\sigma$ and $\left[x_{K}, x_{L}\right]$ are included in the diamonds. The segment $\sigma^{*}=\left[x_{K}, x_{L}\right]$ is actually an edge of the direct dual mesh and a diamond cell is thus associated to a couple of a primal edge $\sigma$ and a dual edge $\sigma^{*}$ that intersect. These edges are the diagonals of the diamond cell which is naturally denoted $D_{\sigma}$, $D_{\sigma^{*}}$, or $D_{\sigma, \sigma^{*}}$ when we want to specify the primal or dual edges associated to the diamond cell.

- or we work with the barycentric dual mesh. We refer the reader to Fig. 12 for an example where there are non convex diamond cells. In this situation, considering a diamond cell, the diagonal $\sigma$ is still included in the diamond while the segment $\left[x_{K}, x_{L}\right]$, possibly not included in the diamond, is not anymore an edge of the dual mesh. By convention, the diamonds are still denoted $D_{\sigma}, D_{\sigma^{*}}$ or $D_{\sigma, \sigma^{*}}$ with $\sigma^{*}=\left[x_{K}, x_{L}\right]$ but there are actually two edges of the dual mesh, denoted $\sigma_{K}^{*}$ and $\sigma_{L}^{*}$, which belong to this diamond. 


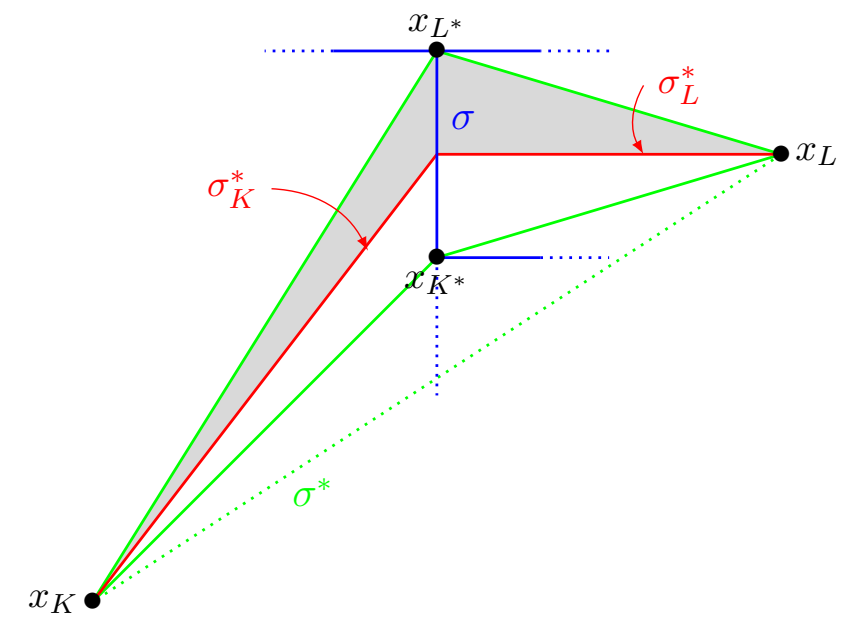

Figure 12: A case of non convex diamond cells: there are two edges of the dual mesh included in the diamond cell. The shaded area is $D_{\sigma, \sigma^{*}} \cap L^{*}$

The formalism previously introduced takes into account both situations and allows to retrieve the standard DDFV discrete operators from definitions (17) and (19) as follows. Let us first consider the primal mesh $\mathfrak{T}$ and the diamond mesh $\mathfrak{D}$ together. There is a one to one application between the cells of $\mathfrak{D}$ and the edges $\sigma$ of $\mathfrak{T}$. Thus, the notation $\mathfrak{S}$ may be understood as a single whole edge $\sigma$ and the cells of $\mathfrak{D}$ can be denoted $D_{\sigma}$. We denote by $\operatorname{div}^{K}$ the divergence operator defined by (19) and $\nabla^{D_{\sigma}}$ the gradient operator defined by (17). Concerning the boundary, the diamond cells of $\mathfrak{D}^{\text {ext }}$ are always included in a primal cell, so that we are in the case where $\mathfrak{D}^{\text {ext, } \partial}=\mathfrak{D}^{\text {ext }}$ and $\mathfrak{D}^{\text {ext, } \not}=\emptyset$. Similarly, we can also consider the dual mesh $\mathfrak{T}^{*}$ and the diamond mesh $\mathfrak{D}$ together. For direct dual meshes, there is a one to one application between the cells of $\mathfrak{D}$ and the edges $\sigma^{*}$ of $\mathfrak{T}^{*}$. The notation $\mathfrak{S}$ may be understood as a single whole edge $\sigma^{*}$. For barycentric dual meshes, the notation $\mathfrak{S}$ stands for $\sigma^{*}=\left[x_{K}, x_{L}\right]$ and allows to gather the two dual edges $\sigma_{K}^{*}$ and $\sigma_{L}^{*}$. As explained before, for both the direct or barycentric dual meshes, we use the notation $D_{\sigma^{*}}$ to denote the cells of $\mathfrak{D}$. Thus, the divergence operator defined by (19) is denoted $\operatorname{div}^{K^{*}}$ and the gradient operator defined by (17) is denoted $\nabla^{D_{\sigma^{*}}}$. Concerning the boundary, the cells of $\mathfrak{D}^{\text {ext }}$ intersect two dual cells, so that we are now in the case where $\mathfrak{D}^{\text {ext, }, \partial}=\emptyset$ and $\mathfrak{D}^{\text {ext, } \not}=\mathfrak{D}^{\text {ext }}$.

The discrete divergence operators $\operatorname{div}^{K}$ and $\operatorname{div}^{K^{*}}$ on the meshes $\mathfrak{T}$ and $\mathfrak{T}^{*}$ are exactly the DDFV divergence operators whereas the DDFV gradient operator $\nabla^{D_{\sigma, \sigma^{*}}}$ on the mesh $\mathfrak{D}$ can be recovered as follows

$$
\begin{aligned}
\nabla^{D_{\sigma, \sigma^{*}}} \Psi^{\mathfrak{T}, \mathfrak{T}^{*}} & =\frac{1}{2\left|D_{\sigma, \sigma^{*}}\right|}\left(|\sigma|\left(\Psi_{L}-\Psi_{K}\right) \mathbf{n}_{K, \sigma}+\left|\sigma^{*}\right|\left(\Psi_{L^{*}}-\Psi_{K^{*}}\right) \mathbf{n}_{K^{*}, \sigma^{*}}\right) \\
& =\frac{1}{2}\left(\nabla^{D_{\sigma}} \Psi^{\mathfrak{T}}+\nabla^{D_{\sigma^{*}}} \Psi^{\mathfrak{T}^{*}}\right)
\end{aligned}
$$

where $\Psi^{\mathfrak{T}}=\left(\Psi_{K}\right)_{K \in \mathfrak{T}}$ (resp. $\left.\Psi^{\mathfrak{T}^{*}}=\left(\Psi_{K^{*}}\right)_{K^{*} \in \mathfrak{T}^{*}}\right)$ is a scalar field defined on the primal mesh (resp. dual mesh) and $\Psi^{\mathfrak{T}, \mathfrak{T}^{*}}=\left(\Psi^{\mathfrak{T}}, \Psi^{\mathfrak{T}^{*}}\right)$ is a DDFV scalar field. 


\subsubsection{Local discrete duality relations}

We now turn to prove discrete equivalents of (1) on a cell $\mathscr{O}=K \in \mathfrak{T}$ or $\mathscr{O}=D_{\mathfrak{S}} \in$ $\mathfrak{D}$. Since the unknowns and the discrete operators are defined on different meshes, these formula will involve local average quantities. We present four discrete versions: in Proposition 3.6 the discrete counterparts of the term $\int_{\mathscr{O}} \operatorname{div} \mathbf{u} \phi \mathrm{d} x$ make use of the discrete operators naturally defined on $\mathscr{O}$ whereas local averages are used in the discrete counterparts of the term $\int_{\mathscr{O}} \mathbf{u} \cdot \nabla \phi \mathrm{d} x$; the other way around is presented in Proposition 3.7. These two propositions state the existence of fluxes which represent $\phi \mathbf{u} \cdot \mathbf{n}$ on the boundary of $\mathscr{O}$ (with $\mathbf{n}$ the outward normal to $\partial \mathscr{O}$ ).

Proposition 3.6. 1) Let a vector field $\mathbf{u}^{\mathfrak{T}}=\left(\mathbf{u}_{K}\right)_{K \in \mathfrak{T}}$ and a scalar field $\Phi^{\mathfrak{D}}=$ $\left(\Phi_{D_{\mathfrak{S}}}\right)_{D_{\mathfrak{S}} \in \mathfrak{D}}$. There exist conservative fluxes $H_{D_{\mathfrak{S}, \mathfrak{s}}}\left(\right.$ for all $\left.D_{\mathfrak{S}} \in \mathfrak{D}, \mathfrak{s} \in \mathscr{E}^{D_{\mathfrak{S}}}\right)$ such that

$$
\sum_{K \in \mathfrak{T}}\left|K \cap D_{\mathfrak{S}}\right|\left(\mathbf{u}_{K} \cdot \nabla^{K} \Phi^{\mathfrak{D}}\right)+\left|D_{\mathfrak{S}}\right| \Phi_{D_{\mathfrak{S}}} \operatorname{div}^{D_{\mathfrak{S}}} \mathbf{u}^{\mathfrak{T}}=\sum_{\mathfrak{s} \in \mathscr{E}^{D} \mathfrak{S}} H_{D_{\mathfrak{S}, \mathfrak{F}}}, \quad \forall D_{\mathfrak{S}} \in \mathfrak{D} .
$$

and

- for all $D_{\mathfrak{S}} \in \mathfrak{D}^{\mathrm{ext}, \not \supset}, \mathfrak{S}=K_{1} \mid K_{2}$,

$$
\sum_{\mathfrak{s} \in \mathscr{E}^{D_{\mathfrak{S}} \cap \mathscr{E}^{\mathfrak{D}, \text { ext }}}} H_{D_{\mathfrak{S}, \mathfrak{s}}}=\left|\mathfrak{S}^{\partial, K_{1}}\right| \Phi_{D_{\mathfrak{S}}} \mathbf{u}_{K_{1}} \cdot \mathbf{n}_{K_{1}, \mathfrak{S}^{\partial, K_{1}}}+\left|\mathfrak{S}^{\partial, K_{2}}\right| \Phi_{D_{\mathfrak{S}}} \mathbf{u}_{K_{2}} \cdot \mathbf{n}_{K_{2}, \mathfrak{S}^{\partial, K_{2}}}
$$

- for all $D_{\mathfrak{S}} \in \mathfrak{D}^{\text {ext, } \partial}, \mathfrak{S}=K_{1} \mid K_{2}$,

$$
\sum_{\mathfrak{s} \in \mathscr{E} D_{\mathfrak{S} \cap \mathscr{E} \mathfrak{Q}, \text { ext }}} H_{D_{\mathfrak{S}, \mathfrak{s}}}=|\mathfrak{S}| \Phi_{D_{\mathfrak{S}}} \mathbf{u}_{K_{2}} \cdot \mathbf{n}_{K_{1}, \mathfrak{S}}
$$

2) Let a scalar field $\Psi^{\mathfrak{T}}=\left(\Psi_{K}\right)_{K \in \mathfrak{T}}$ and a vector field $\mathbf{v}^{\mathfrak{D}}=\left(\mathbf{v}_{D_{\mathfrak{S}}}\right)_{D_{\mathfrak{S}} \in \mathfrak{D}}$. There exist conservative fluxes $H_{K, \sigma}$ (for all $K \in \mathfrak{T}, \sigma \in \mathscr{E}^{K}$ ) such that

$$
\sum_{D_{\mathfrak{S}} \in \mathfrak{D}}\left|K \cap D_{\mathfrak{S}}\right|\left(\mathbf{v}_{D_{\mathfrak{S}}} \cdot \nabla^{D_{\mathfrak{S}}} \Psi^{\mathfrak{T}}\right)+|K| \Psi_{K} \operatorname{div}^{K} \mathbf{v}^{\mathfrak{D}}=\sum_{\sigma \in \mathscr{E} K} H_{K, \sigma}, \quad \forall K \in \mathfrak{T},
$$

and, for all $\sigma \in \mathscr{E}^{\mathfrak{T}, \text { ext }}, \sigma \in \mathscr{E}^{K}$,

$$
\begin{aligned}
H_{K, \sigma}=\left(\sum_{\substack{D_{\mathfrak{S}} \in \mathfrak{D}^{\sigma} \cap \mathfrak{D}^{\text {ext }, 2} \\
\mathfrak{S}=K \mid L}}\left|\sigma \cap D_{\mathfrak{S}}\right| \Psi_{K} \mathbf{v}_{D_{\mathfrak{S}}}\right. \\
\left.+\sum_{\substack{D_{\mathfrak{S}} \in \mathfrak{D}^{\sigma} \cap \mathfrak{D}^{\operatorname{ext}, \boldsymbol{B}} \\
\mathfrak{S}=K \mid L}}\left|\sigma \cap D_{\mathfrak{S}}\right| \Psi_{L} \mathbf{v}_{D_{\mathfrak{S}}}\right) \cdot \mathbf{n}_{K, \sigma} .
\end{aligned}
$$

Proof. We begin with the proof of (20), (21) and (22). We shall make use of the definition of the discrete operators in (19). We remark that

$$
\mathbf{u}_{K} \cdot \nabla^{K} \Phi^{\mathfrak{D}}=\frac{1}{|K|}\left(\sum_{D_{\mathfrak{S}} \in \mathfrak{D}^{K}} \mathbf{u}_{K} \cdot \mathbf{F}_{K, \mathfrak{S}}+\sum_{D_{\mathfrak{S}} \in \mathfrak{D}^{K, \nexists}} \mathbf{u}_{K} \cdot \mathbf{F}_{K, \mathfrak{S}^{\partial, K}}\right)
$$

Corollary 3.4 gives the existence of conservative fluxes $\widetilde{H}_{D_{\mathfrak{S}, \mathfrak{s}}}$ such that 
- for all $D_{\mathfrak{S}} \in \mathfrak{D}^{\text {int }} \cup \mathfrak{D}^{\text {ext, }, \not}$,

$$
\sum_{\mathfrak{s} \in \mathscr{E}^{D} \mathfrak{S}} \widetilde{H}_{D_{\mathfrak{S}, \mathfrak{S}}}=\sum_{K \in \mathfrak{T}}\left|K \cap D_{\mathfrak{S}}\right|\left(\mathbf{u}_{K} \cdot \nabla^{K} \Phi^{\mathfrak{D}}\right)-\left(\mathbf{u}_{K_{1}} \cdot \mathbf{F}_{K_{1}, \mathfrak{S}}+\mathbf{u}_{K_{2}} \cdot \mathbf{F}_{K_{2}, \mathfrak{S}}\right)
$$

- for all $D_{\mathfrak{S}} \in \mathfrak{D}^{\mathrm{ext}, \partial}$,

$$
\sum_{\mathfrak{s} \in \mathscr{E}^{D} D_{\mathfrak{S}}} \widetilde{H}_{D_{\mathfrak{S}, \mathfrak{F}}}=\sum_{K \in \mathfrak{T}}\left|K \cap D_{\mathfrak{S}}\right|\left(\mathbf{u}_{K} \cdot \nabla^{K} \Phi^{\mathfrak{D}}\right) .
$$

Moreover, on the boundary of the domain we have

- for all $D_{\mathfrak{S}} \in \mathfrak{D}^{\text {ext, } \not \supset}, \mathfrak{S}=K_{1} \mid K_{2}$,

$$
\sum_{\mathfrak{s} \in \mathscr{E}^{D}{ }_{\mathfrak{S} \cap \mathscr{E}^{\mathfrak{D}, \text { ext }}}} \widetilde{H}_{D_{\mathfrak{S}, \mathfrak{s}}}=\mathbf{u}_{K_{1}} \cdot \mathbf{F}_{K_{1}, \mathfrak{S}^{\partial, K_{1}}}+\mathbf{u}_{K_{2}} \cdot \mathbf{F}_{K_{2}, \mathfrak{S}^{\partial, K_{2}}}
$$

- for all $D_{\mathfrak{S}} \in \mathfrak{D}^{\text {ext, } \partial}, \mathfrak{S}=K_{1} \mid K_{2}$,

$$
\sum_{\mathfrak{s} \in \mathscr{E}^{D}{ }_{\mathfrak{S} \cap \mathscr{E}^{\mathfrak{D}, \text { ext }}}} \widetilde{H}_{D_{\mathfrak{S}, \mathfrak{s}}}=\mathbf{u}_{K_{1}} \cdot \mathbf{F}_{K_{1}, \mathfrak{S}} \cdot
$$

However, for all $D_{\mathfrak{S}} \in \mathfrak{D}^{\text {int }} \cup \mathfrak{D}^{\text {ext, } \not \supset}, \mathfrak{S}=K_{1} \mid K_{2}$, by definition and owing to (15), we have

$$
\mathbf{u}_{K_{1}} \cdot \mathbf{F}_{K_{1}, \mathfrak{S}}+\mathbf{u}_{K_{2}} \cdot \mathbf{F}_{K_{2}, \mathfrak{S}}=|\mathfrak{S}| \Phi_{D_{\mathfrak{S}}}\left(\mathbf{u}_{K_{1}}-\mathbf{u}_{K_{2}}\right) \cdot \mathbf{n}_{K_{1}, \mathfrak{S}}
$$

Owing to Lemma 3.5 , for all $D_{\mathfrak{S}} \in \mathfrak{D}^{\text {int }} \cup \mathfrak{D}^{\text {ext, } \not \mathscr{\phi}}, \mathfrak{S}=K_{1} \mid K_{2}$, we arrive at

$$
\mathbf{u}_{K_{1}} \cdot \mathbf{F}_{K_{1}, \mathfrak{S}}+\mathbf{u}_{K_{2}} \cdot \mathbf{F}_{K_{2}, \mathfrak{S}}=-\left|D_{\mathfrak{S}}\right| \Phi_{D_{\mathfrak{S}}} \operatorname{div}^{D_{\mathfrak{S}}} \mathbf{u}^{\mathfrak{T}}
$$

Thus we can define $H_{D_{\mathfrak{S}, \mathfrak{s}}}=\widetilde{H}_{D_{\mathfrak{S}, \mathfrak{s}}}$ for all $D_{\mathfrak{S}} \in \mathfrak{D}^{\text {int }} \cup \mathfrak{D}^{\text {ext, } \not}$, for all $\mathfrak{s} \in \mathscr{E}^{D_{\mathfrak{S}}}$ but also for all $D_{\mathfrak{S}} \in \mathfrak{D}^{\text {ext, } \partial}$, for all $\mathfrak{s} \in \mathscr{E}^{D_{\mathfrak{S}}} \cap \mathscr{E}^{\mathfrak{D} \text {,int }}$. We modify the boundary fluxes so that

$$
\sum_{\mathfrak{s} \in \mathscr{E}^{D}{ }_{\mathfrak{S} \cap \mathscr{E}^{\mathfrak{D}, \text { ext }}}} H_{D_{\mathfrak{S}, \mathfrak{s}}}=\sum_{\mathfrak{s} \in \mathscr{E}^{D} \mathfrak{S} \cap \mathscr{E}_{\mathfrak{D}, \text { ext }}} \widetilde{H}_{D_{\mathfrak{S}, \mathfrak{s}}}-\left(\mathbf{u}_{K_{1}}-\mathbf{u}_{K_{2}}\right) \cdot \mathbf{F}_{K_{1}, \mathfrak{S}}, \quad \forall D_{\mathfrak{S}} \in \mathfrak{D}^{\mathrm{ext}, \partial}, \mathfrak{S}=K_{1} \mid K_{2} .
$$

For instance, for $D_{\mathfrak{G}} \in \mathfrak{D}^{\text {ext, }, \partial}, \mathfrak{S}=K_{1} \mid K_{2}$, the modified fluxes can be obtained by substracting the term $\left(\mathbf{u}_{K_{1}}-\mathbf{u}_{K_{2}}\right) \cdot \mathbf{F}_{K_{1}, \mathfrak{S}}$ to an arbitrarily selected boundary flux, the others remaining unchanged. The conservativity of the fluxes $H_{D_{\mathfrak{S}, \mathfrak{s}}}$ directly comes from the conservativity of the fluxes $\widetilde{H}_{D_{\mathfrak{S}, \mathfrak{s}}}$ since we only modify boundary fluxes (each $\mathfrak{s} \in \mathscr{E}^{\mathfrak{D}, \text { ext }}$ belongs to only one cell $\left.D_{\mathfrak{S}} \in \mathfrak{D}\right)$. We can then readily verify that equalities (20), (21) and (22) hold as a consequence of the definition of the fluxes $H_{D_{\mathfrak{S}, \mathfrak{s}}}$ and of equalities $(25),(26)$ and $(27)$.

We now turn to the proof of (23). The result is obtained by applying Theorem 2.7 while reversing the role of $\mathfrak{T}$ and $\mathfrak{D}$. An edge $\mathfrak{s}$ of a cell $D_{\mathfrak{S}} \in \mathfrak{D}^{\text {int }} \cup \mathfrak{D}^{\text {ext, }, \boldsymbol{D}}, \mathfrak{S}=K_{1} \mid K_{2}$, is splitted at most in two parts: $\mathfrak{s}^{K_{1}}$ and $\mathfrak{s}^{K_{2}}$. Since we have the following equality

$$
\begin{aligned}
\mathbf{v}_{D_{\mathfrak{S}}} \cdot \nabla^{D_{\mathfrak{S}}} \Psi^{\mathfrak{T}} & =\frac{1}{\left|D_{\mathfrak{S}}\right|} \sum_{\mathfrak{s} \in \mathscr{E} D_{\mathfrak{S}}} \mathbf{v}_{D_{\mathfrak{S}}} \cdot \mathbf{G}_{D_{\mathfrak{S}, \mathfrak{S}}} \\
& =\frac{1}{\left|D_{\mathfrak{S}}\right|} \sum_{\mathfrak{s} \in \mathscr{E}^{D} \mathfrak{S}}\left(\left|\mathfrak{s}^{K_{1}}\right| \Psi_{K_{1}} \mathbf{v}_{D_{\mathfrak{S}}} \cdot \mathbf{n}_{D_{\mathfrak{S}, \mathfrak{F}}}+\left|\mathfrak{s}^{K_{2}}\right| \Psi_{K_{2}} \mathbf{v}_{D_{\mathfrak{S}}} \cdot \mathbf{n}_{D_{\mathfrak{S}, \mathfrak{s}}}\right)
\end{aligned}
$$


it is natural to write

$$
\mathbf{v}_{D_{\mathfrak{S}}} \cdot \nabla^{D_{\mathfrak{S}}} \Psi^{\mathfrak{T}}=\frac{1}{\left|D_{\mathfrak{S}}\right|} \sum_{\mathfrak{s} \in \mathscr{E}^{D} D_{\mathfrak{S}}}\left(\mathscr{H}_{D_{\mathfrak{S}, \mathfrak{F}} K_{1}}+\mathscr{H}_{D_{\mathfrak{S}, \mathfrak{s}} K_{2}}\right)
$$

where the fluxes $\mathscr{H}_{D_{\mathfrak{S}, \mathfrak{s}} K_{i}}$ are defined by

$$
\mathscr{H}_{D_{\mathfrak{S}, \mathfrak{s}} K_{i}}=\left|\mathfrak{s}^{K}\right| \Psi_{K_{i}} \mathbf{v}_{D_{\mathfrak{S}}} \cdot \mathbf{n}_{D_{\mathfrak{S}, \mathfrak{s}}} \cdot
$$

Then, Theorem 2.7 leads to the existence of conservative fluxes $H_{K, \sigma}$ such that

$$
\sum_{\sigma \in \mathscr{E}^{K}} H_{K, \sigma}=\sum_{D_{\mathfrak{S}} \in \mathfrak{D}}\left|D_{\mathfrak{S}} \cap K\right|\left(\mathbf{v}_{D_{\mathfrak{S}}} \cdot \nabla^{D_{\mathfrak{S}}} \Psi^{\mathfrak{T}}\right)-\sum_{D_{\mathfrak{S}} \in \mathfrak{D}^{K}} \sum_{\substack{\mathfrak{s} \in \mathscr{C}^{D} D_{\mathfrak{S}} \\ \mathfrak{s}^{K} \not \subset \partial X}} \mathscr{H}_{D_{\mathfrak{S}, \mathfrak{F}^{K}}}, \quad \forall K \in \mathfrak{T} .
$$

It remains to identify the second term in the right hand side

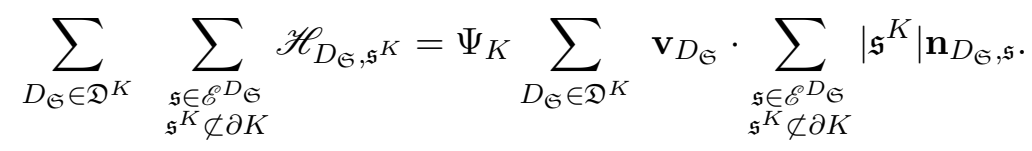

We now remark that:

- if $D_{\mathfrak{S}} \in \mathfrak{D}^{K} \cap \mathfrak{D}^{\text {int }}$ and $\mathfrak{s}^{K} \in \mathscr{E}^{D_{\mathfrak{S}}}$ then $\mathfrak{s}$ cannot belong to $\partial K$ since interior edges of $\mathfrak{T}$ and $\mathfrak{D}$ do not overlap by Assumption 2.6.

- if $D_{\mathfrak{S}} \in \mathfrak{D}^{K} \cap \mathfrak{D}^{\text {ext, } \partial}$ and $\mathfrak{s} \in \mathscr{E}^{D_{\mathfrak{S}}}$ then $\mathfrak{s}^{K} \subset \partial K$ implies that $\mathfrak{s}^{K}=\emptyset$ since by convention boundary edges are associated to the exterior fictitious cell in this case.

Thus we can write

$$
\begin{aligned}
& \sum_{D_{\mathfrak{S} \in \mathfrak{D}^{K}}} \sum_{\substack{\mathfrak{s} \in \mathscr{E}^{D} D_{\mathfrak{S}} \\
\mathfrak{s}^{K} \not \subset \partial K}} \mathscr{H}_{D_{\mathfrak{S}, \mathfrak{s}} K}=\Psi_{K} \sum_{D_{\mathfrak{S}} \in \mathfrak{D}^{K}} \mathbf{v}_{D_{\mathfrak{S}}} \cdot \sum_{\mathfrak{s} \in \mathscr{E}^{D} \mathfrak{S}_{\mathfrak{S}}}\left|\mathfrak{s}^{K}\right| \mathbf{n}_{D_{\mathfrak{S}, \mathfrak{s}}} \\
& -\Psi_{K} \sum_{D_{\mathfrak{S}} \in \mathfrak{D}^{K, \phi}} \mathbf{v}_{D_{\mathfrak{S}}} \cdot \sum_{\substack{\mathfrak{s} \in \mathscr{E}^{D_{\mathfrak{S}}} \\
\mathfrak{s}^{K} \subset \partial K}}\left|\mathfrak{s}^{K}\right| \mathbf{n}_{D_{\mathfrak{S}, \mathfrak{F}}} \cdot
\end{aligned}
$$

Owing to (18), we have

$$
\sum_{\mathfrak{s} \in \mathscr{E}^{D} \mathfrak{S}}\left|\mathfrak{s}^{K}\right| \mathbf{n}_{D_{\mathfrak{S}, \mathfrak{S}}}=-|\mathfrak{S}| \mathbf{n}_{K, \mathfrak{S}},
$$

and since $\left\{\mathfrak{s}^{K} ; \mathfrak{s} \in \mathscr{E}^{D_{\mathfrak{S}}}, \mathfrak{s} \subset \partial K\right\}=\left\{\sigma^{D} ; \sigma^{D} \in \mathfrak{E}_{\mathfrak{D}}^{K} \cap \mathfrak{E}_{\mathfrak{D}}^{\mathfrak{T}, \text { ext }}, \sigma^{D} \subset D_{\mathfrak{S}}\right\}$ when $D_{\mathfrak{S}} \in$ $\mathfrak{D}^{K, \not \supset}$, we have, by definition,

$$
\sum_{\substack{\mathfrak{s} \in \mathscr{E}^{\mathscr{D}} \mathfrak{S}_{\mathfrak{S}} \\ \mathfrak{s}^{K} \subset \partial K}}\left|\mathfrak{s}^{K}\right| \mathbf{n}_{D_{\mathfrak{S}, \mathfrak{s}}}=\left|\mathfrak{S}^{\partial, K}\right| \mathbf{n}_{K, \mathfrak{S}^{\partial, K}}
$$


We arrive at

$$
\begin{aligned}
\sum_{D_{\mathfrak{S}} \in \mathfrak{D}^{K}} \sum_{\substack{\mathfrak{s} \in \mathscr{E}^{D_{\mathfrak{S}}} \\
\mathfrak{s}^{K} \not \subset \partial K}} \mathscr{H}_{D_{\mathfrak{S}, \mathfrak{s}} K} & =-\Psi_{K} \sum_{D_{\mathfrak{S}} \in \mathfrak{D}^{K}}|\mathfrak{S}| \mathbf{v}_{D_{\mathfrak{S}}} \cdot \mathbf{n}_{K, \mathfrak{S}}-\Psi_{K} \sum_{D_{\mathfrak{S}} \in \mathfrak{D}^{K, \phi}}\left|\mathfrak{S}^{\partial, K}\right| \mathbf{v}_{D_{\mathfrak{S}}} \cdot \mathbf{n}_{K, \mathfrak{S}^{\partial, K}} \\
& =-|K| \Psi_{K} \operatorname{div}^{K} \mathbf{v}^{\mathfrak{D}} .
\end{aligned}
$$

This gives the conclusion. The expression of the fluxes on the boundary comes directly from the corresponding result in Theorem 2.7.

We can also prove the following equivalent duality result which is obtained by reversing the role of the divergence and gradient operators.

Proposition 3.7. 1) Let a scalar field $\Psi^{\mathfrak{T}}=\left(\Psi_{K}\right)_{K \in \mathfrak{T}}$ and a vector field $\mathbf{v}^{\mathfrak{D}}=$ $\left(\mathbf{v}_{D_{\mathfrak{S}}}\right)_{D_{\mathfrak{S}} \in \mathfrak{D}}$. There exist conservative fluxes $H_{D_{\mathfrak{S}, \mathfrak{s}}}\left(\right.$ for all $\left.D_{\mathfrak{S}} \in \mathfrak{D}, \mathfrak{s} \in \mathscr{E}^{D_{\mathfrak{S}}}\right)$ such that

$$
\sum_{K \in \mathfrak{T}}\left|K \cap D_{\mathfrak{S}}\right|\left(\Psi_{K} \operatorname{div}^{K} \mathbf{v}^{\mathfrak{D}}\right)+\left|D_{\mathfrak{S}}\right| \mathbf{v}_{D_{\mathfrak{S}}} \cdot \nabla^{D_{\mathfrak{S}}} \Psi^{\mathfrak{T}}=\sum_{\mathfrak{s} \in \mathscr{E}^{D_{\mathfrak{S}}}} H_{D_{\mathfrak{S}, \mathfrak{s}}}, \quad \forall D_{\mathfrak{S}} \in \mathfrak{D},
$$

and

- for all $D_{\mathfrak{S}} \in \mathfrak{D}^{\operatorname{ext}, \not \supset}, \mathfrak{S}=K_{1} \mid K_{2}$,

$$
\sum_{\mathfrak{s} \in \mathscr{E}^{D} D_{\mathfrak{S} \cap \mathscr{E}} \mathcal{D}, \text { ext }} H_{D_{\mathfrak{S}, \mathfrak{F}}}=\left|\mathfrak{S}^{\partial, K_{1}}\right| \Psi_{K_{1}} \mathbf{v}_{D_{\mathfrak{S}}} \cdot \mathbf{n}_{K_{1}, \mathfrak{S}^{\partial, K_{1}}}+\left|\mathfrak{S}^{\partial, K_{2}}\right| \Psi_{K_{2}} \mathbf{v}_{D_{\mathfrak{S}}} \cdot \mathbf{n}_{K_{2}, \mathfrak{S}^{\partial, K_{2}}},
$$

- for all $D_{\mathfrak{S}} \in \mathfrak{D}^{\mathrm{ext}, \partial}, \mathfrak{S}=K_{1} \mid K_{2}$,

$$
\sum_{\mathfrak{s} \in \mathscr{E}^{D} D_{\mathfrak{S} \cap \mathscr{E} \mathfrak{D}, \text { ext }}} H_{D_{\mathfrak{S}, \mathfrak{s}}}=|\mathfrak{S}| \Psi_{K_{2}} \mathbf{v}_{D_{\mathfrak{S}}} \cdot \mathbf{n}_{K_{1}, \mathfrak{G}} \cdot
$$

2) Let a vector field $\mathbf{u}^{\mathfrak{T}}=\left(\mathbf{u}_{K}\right)_{K \in \mathfrak{T}}$ and a scalar field $\Phi^{\mathfrak{D}}=\left(\Phi_{D_{\mathfrak{S}}}\right)_{D_{\mathfrak{S}} \in \mathfrak{D}}$. There exist conservative fluxes $H_{K, \sigma}$ (for all $K \in \mathfrak{T}, \sigma \in \mathscr{E}^{K}$ ) such that

$$
\sum_{D_{\mathfrak{S}} \in \mathfrak{D}}\left|K \cap D_{\mathfrak{S}}\right|\left(\Phi_{D_{\mathfrak{S}}} \operatorname{div}^{D_{\mathfrak{S}}} \mathbf{u}^{\mathfrak{T}}\right)+|K| \mathbf{u}_{K} \cdot \nabla^{K} \Phi^{\mathfrak{D}}=\sum_{\sigma \in \mathscr{E} K} H_{K, \sigma}, \quad \forall K \in \mathfrak{T}
$$

and, for all $\sigma \in \mathscr{E}^{\mathfrak{T}, \text { ext }}, \sigma \in \mathscr{E}^{K}$,

$$
\begin{aligned}
H_{K, \sigma}=\left(\sum_{\substack{D_{\mathfrak{S}} \in \mathfrak{D}^{\sigma} \cap \mathfrak{D}^{\text {ext }, \partial} \\
\mathfrak{S}=K \mid L}}\left|\sigma \cap D_{\mathfrak{S}}\right| \Phi_{D_{\mathfrak{S}}} \mathbf{u}_{K}\right. \\
\left.+\sum_{\substack{D_{\mathfrak{S}} \in \mathfrak{D}^{\sigma} \cap \mathfrak{D}^{\text {ext }, \not} \\
\mathfrak{S}=K \mid L}}\left|\sigma \cap D_{\mathfrak{S}}\right| \Phi_{D_{\mathfrak{S}}} \mathbf{u}_{L}\right) \cdot \mathbf{n}_{K, \sigma} .
\end{aligned}
$$


Proof. Proposition 3.7 is obtained by applying Proposition 3.6 twice. We denote $\mathbf{e}_{1}=$ $(1,0) \in \mathbb{R}^{2}$ and $\mathbf{e}_{2}=(0,1) \in \mathbb{R}^{2}$. Set $\mathbf{u}_{K}^{(i)}=\Psi_{K} \mathbf{e}_{i}$ and $\Phi_{D_{\mathfrak{S}}}^{(i)}=\mathbf{v}_{D_{\mathfrak{S}}} \cdot \mathbf{e}_{i}$. We observe that

$$
\sum_{i=1}^{2} \mathbf{e}_{i} \cdot \nabla^{K} \Phi^{\mathfrak{D},(i)}=\operatorname{div}^{K} \mathbf{v}^{\mathfrak{D}}
$$

and

$$
\operatorname{div}^{D_{\mathfrak{S}}} \mathbf{u}^{\mathfrak{T},(i)}=\nabla^{D_{\mathfrak{S}}} \Psi^{\mathfrak{T}} \cdot \mathbf{e}_{i}
$$

hold. We then apply Proposition 3.6 twice for $i=1$ and 2 and sum the obtained results. Equations (28), (29) and (30) are readily deduced from (20), (21) and (22) respectively.

In the same vein, we can prove (31) and (32) by setting $\mathbf{v}_{D_{\mathfrak{S}}}=\Phi_{D_{\mathfrak{S}}} \mathbf{e}_{i}$ and $\Psi_{K}=$ $\mathbf{u}_{K} \cdot \mathbf{e}_{i}$.

Two-point flux approximation (TPFA) Proposition 3.6 and 3.7 give local discrete duality relations between these two operators through equations (23) and (28), which can be seen as discrete counterparts of the equality stated in (Q2). Of course, the local duality equations imply the following more standard global discrete duality relation

$$
\sum_{D_{\sigma} \in \mathfrak{D}}\left|D_{\sigma}\right| \mathbf{v}_{D_{\sigma}} \cdot \nabla^{D_{\sigma}} \Psi^{\mathfrak{T}}+\sum_{K \in \mathfrak{T}}|K| \Psi_{K} \operatorname{div}^{K} \mathbf{v}^{\mathfrak{D}}=\sum_{\substack{\sigma \in \mathscr{E} \text { ext } \\ \sigma=K \mid L}}|\sigma| \Psi_{L} \mathbf{v}_{D_{\sigma}} \cdot \mathbf{n}_{K, \sigma} .
$$

Discrete duality finite volume discretization (DDFV) Proposition 3.7 allows us to justify the following local discrete duality relations, for all $D_{\sigma} \in \mathfrak{D}$,

$$
\sum_{K \in \mathfrak{T}}\left|K \cap D_{\sigma}\right|\left(\Psi_{K} \operatorname{div}^{K} \mathbf{v}^{\mathfrak{D}}\right)+\left|D_{\sigma}\right| \mathbf{v}_{D_{\sigma}} \cdot \nabla^{D_{\sigma}} \Psi^{\mathfrak{T}}=\sum_{\mathfrak{s} \in \mathscr{E}^{D}} H_{D_{\sigma}, \mathfrak{s}}
$$

and, for all $D_{\sigma^{*}} \in \mathfrak{D}$,

$$
\sum_{K^{*} \in \mathfrak{T}^{*}}\left|K^{*} \cap D_{\sigma^{*}}\right|\left(\Psi_{K^{*}} \operatorname{div}^{K^{*}} \mathbf{v}^{\mathfrak{D}}\right)+\left|D_{\sigma^{*}}\right| \mathbf{v}_{D_{\sigma^{*}}} \cdot \nabla^{D_{\sigma^{*}}} \Psi^{\mathfrak{T}^{*}}=\sum_{\mathfrak{s} \in \mathscr{E}^{D_{\sigma^{*}}}} H_{D_{\sigma^{*}, \mathfrak{s}}^{*}} .
$$

Summing up these two relations gives a local duality relation for the discrete DDFV operators

$$
\begin{aligned}
& \frac{1}{2}\left(\sum_{K \in \mathfrak{T}} \mid K\right.\left.\cap D_{\sigma}\left|\left(\Psi_{K} \operatorname{div}^{K} \mathbf{v}^{\mathfrak{D}}\right)+\sum_{K^{*} \in \mathfrak{T}^{*}}\right| K^{*} \cap D_{\sigma^{*}} \mid\left(\Psi_{K^{*}} \operatorname{div}^{K^{*}} \mathbf{v}^{\mathfrak{D}}\right)\right) \\
&+\left|D_{\sigma, \sigma^{*}}\right| \mathbf{v}_{D_{\sigma, \sigma^{*}}} \cdot \nabla^{D_{\sigma, \sigma^{*}}} \Psi^{\mathfrak{T}, \mathfrak{T}^{*}}=\sum_{\mathfrak{s} \in \mathscr{E}^{D_{\sigma, \sigma^{*}}}} \frac{H_{D_{\sigma, \mathfrak{s}}}+H_{D_{\sigma^{*}, \mathfrak{s}}^{*}}}{2}, \quad \forall D_{\sigma, \sigma^{*}} \in \mathfrak{D} .
\end{aligned}
$$

Obviously, we can also prove global relations from these equalities. A sum of equations (33) over the cells $D_{\sigma} \in \mathfrak{D}$ leads to

$$
\sum_{K \in \mathfrak{T}}|K|\left(\Psi_{K} \operatorname{div}^{K} \mathbf{v}^{\mathfrak{D}}\right)+\sum_{D_{\sigma} \in \mathfrak{D}}\left|D_{\sigma}\right| \mathbf{v}_{D_{\sigma}} \cdot \nabla^{D_{\sigma}} \Psi^{\mathfrak{T}}=\sum_{D_{\sigma} \in \mathfrak{D}^{\mathrm{ext}}}|\sigma| \Psi_{L} \mathbf{v}_{D_{\sigma}} \cdot \mathbf{n}_{K, \sigma}
$$


Indeed, by conservativity, the interior fluxes $H_{D_{\sigma, \mathfrak{s}}}$ vanish whereas the boundary fluxes are given by (30) since $\mathfrak{D}^{\text {ext, } \not \supset}=\emptyset$ in this case. Similarly, a sum of equations (34) over the cells $D_{\sigma^{*}} \in \mathfrak{D}$ leads to

$$
\begin{aligned}
\sum_{K^{*} \in \mathfrak{T}^{*}}\left|K^{*}\right|\left(\Psi_{K^{*}} \operatorname{div} K^{*} \mathbf{v}^{\mathfrak{D}}\right)+\sum_{D_{\sigma^{*} \in \mathfrak{D}}\left|D_{\sigma^{*}}\right| \mathbf{v}_{D_{\sigma^{*}}} \cdot \nabla^{D_{\sigma^{*}}} \Psi^{\mathfrak{T}^{*}}} & =\sum_{D_{\sigma^{*}} \in \mathfrak{D}^{\mathrm{ext}}} \frac{|\sigma|}{2}\left(\Psi_{K^{*}}+\Psi_{L^{*}}\right) \mathbf{v}_{D_{\sigma^{*}}} \cdot \mathbf{n}_{K, \sigma} .
\end{aligned}
$$

Indeed, in this case, we have $\mathfrak{D}^{\text {ext, } \partial}=\emptyset$ so that the boundary fluxes are given by (29). Summing up these two relations we find the well-known global discrete duality for DDFV operators

$$
\begin{aligned}
& \frac{1}{2}\left(\sum_{K \in \mathfrak{T}}|K|\left(\Psi_{K} \operatorname{div}^{K} \mathbf{v}^{\mathfrak{D}}\right)+\sum_{K^{*} \in \mathfrak{T}^{*}}\left|K^{*}\right|\left(\Psi_{K^{*}} \operatorname{div}^{K^{*}} \mathbf{v}^{\mathfrak{D}}\right)\right) \\
& +\sum_{D_{\sigma, \sigma^{*}} \in \mathfrak{D}}\left|D_{\sigma, \sigma^{*}}\right| \mathbf{v}_{D_{\sigma, \sigma^{*}}} \cdot \nabla^{D_{\sigma, \sigma^{*}}} \Psi^{\mathfrak{T}, \mathfrak{T}^{*}} \\
& =\sum_{D_{\sigma, \sigma^{*}} \in \mathfrak{D}^{\mathrm{ext}}}|\sigma| \frac{2 \Psi_{L}+\Psi_{K^{*}}+\Psi_{L^{*}}}{4} \mathbf{v}_{D_{\sigma, \sigma^{*}}} \cdot \mathbf{n}_{K, \sigma} .
\end{aligned}
$$

\section{A Proof of Lemma 2.2 with $H^{1}$ regularity}

In this section, we prove Lemma 2.2 with $H^{1}$ regularity for $\boldsymbol{\omega}_{K}$ (see Remark 2.3). Let us denote by $s_{j}, j \in\{1, . ., n\}$, the $n$ vertices of $K$ listed in order as $\partial K$ is traversed counterclockwise and by $\sigma_{j}$ the open line segment connecting $s_{j-1}$ and $s_{j}$. The subscripts $j$ are interpreted modulo $n$. We first introduce the constant function $f$ defined by

$$
f: x \in K \longmapsto \frac{1}{|K|} \sum_{\sigma \in \mathscr{E} K} \mathcal{X}_{K, \sigma} .
$$

We then consider two functions $\varphi_{0}, \varphi_{1}$ of class $\mathscr{C}^{\infty}$ such that

- $\varphi_{0}$ has a compact support in $\left(s_{j-1}, s_{j}\right)$ and

$$
\int_{\sigma_{j}} \varphi_{0}=1
$$

- $\varphi_{1}$ has a compact support in $\left[s_{j-1}, s_{j}\right)$ and is identically equal to 1 in a neighborhood of $s_{j-1}$.

We define the function $\mathbf{g}_{j}$ on $\sigma_{j}$ by

$$
s \in \sigma_{j} \longmapsto \mathbf{g}_{j}(s)=f\left(s_{j-1}\right)\left\|s-s_{j-1}\right\| \varphi_{1}(s) \boldsymbol{\tau}_{K, \sigma_{j}}+\mathcal{X}_{K, \sigma_{j}} \varphi_{0}(s) \mathbf{n}_{K, \sigma_{j}},
$$


where $\boldsymbol{\tau}_{K, \sigma_{j}}$ stands for the unit vector, tangent to the edge $\sigma_{j}$, and such that $\boldsymbol{\tau}_{K, \sigma_{j}}$. $\mathbf{n}_{K, \sigma_{j}}>0$. We readily verify that, for any $j \in\{1, \ldots, n\}$,

$$
\mathbf{g}_{j}\left(s_{j-1}\right)=0, \quad \mathbf{g}_{j}\left(s_{j}\right)=0, \quad \text { and } \quad \int_{\sigma_{j}} \mathbf{g}_{j} \cdot \mathbf{n}_{K, \sigma_{j}}=\mathcal{X}_{K, \sigma_{j}} .
$$

Moreover we have

$$
\begin{aligned}
\frac{\partial \mathbf{g}_{j}}{\partial \boldsymbol{\tau}_{K, \sigma_{j}}}(s)=f\left(s_{j-1}\right)\left(\varphi_{1}(s)+\| s-\right. & \left.s_{j-1} \| \boldsymbol{\nabla}_{\varphi_{1}}(s) \cdot \boldsymbol{\tau}_{K, \sigma_{j}}\right) \boldsymbol{\tau}_{K, \sigma_{j}} \\
& +\mathcal{X}_{K, \sigma_{j}}\left(\boldsymbol{\nabla} \varphi_{0}(s) \cdot \boldsymbol{\tau}_{K, \sigma_{j}}\right) \mathbf{n}_{K, \sigma_{j}}, \quad \forall s \in \sigma_{j} .
\end{aligned}
$$

Hence, evaluating this expression at $s_{j-1}$ and $s_{j}$ we find

$$
\frac{\partial \mathbf{g}_{j}}{\partial \boldsymbol{\tau}_{K, \sigma_{j}}}\left(s_{j}\right)=0 \quad \text { and } \quad \frac{\partial \mathbf{g}_{j+1}}{\partial \boldsymbol{\tau}_{K, \sigma_{j+1}}}\left(s_{j}\right)=f\left(s_{j}\right) \boldsymbol{\tau}_{K, \sigma_{j+1}} .
$$

We arrive at

$$
\left(\frac{\partial \mathbf{g}_{j+1}}{\partial \boldsymbol{\tau}_{K, \sigma_{j+1}}} \cdot \mathbf{n}_{K, \sigma_{j}}-\frac{\partial \mathbf{g}_{j}}{\partial \boldsymbol{\tau}_{K, \sigma_{j}}} \cdot \mathbf{n}_{K, \sigma_{j+1}}\right)\left(s_{j}\right)=f\left(s_{j}\right) \boldsymbol{\tau}_{K, \sigma_{j+1}} \cdot \mathbf{n}_{K, \sigma_{j}} .
$$

Equations (35) and (36) are exactly the compatibility conditions required to apply [4, Theorem 7.1] which proves the existence of a function $\boldsymbol{\omega}_{K} \in H^{s}(\stackrel{\circ}{K})$, with $s>1$, such that

$$
\left\{\begin{aligned}
\nabla \cdot \boldsymbol{\omega}_{K}=f, & \text { in } \stackrel{\circ}{K}, \\
\boldsymbol{\omega}_{K}=\mathbf{g}, & \text { on } \partial K .
\end{aligned}\right.
$$

The first equation corresponds to equality (2). Equality (3) is satisfied too since, for all $j$,

$$
\int_{\sigma_{j}} \boldsymbol{\omega}_{K} \cdot \mathbf{n}_{K, \sigma_{j}}=\int_{\sigma_{j}} \mathbf{g}_{j} \cdot \mathbf{n}_{K, \sigma_{j}}=\mathcal{X}_{K, \sigma_{j}} .
$$

\section{B Proof of Lemma 2.9}

We denote $P_{1}, P_{2}, \ldots P_{n}$ the $n$ vertices of $K$, numbered clockwise, and $\left(x_{j}, y_{j}\right)$ stands for the coordinates of $P_{j}$. For $P_{i}$ and $P_{j}$ two consecutive vertices of $K$, we set

$$
\left(\begin{array}{c}
a_{i, j} \\
b_{i, j}
\end{array}\right)=\left(\begin{array}{c}
x_{j}-x_{i} \\
y_{j}-y_{i}
\end{array}\right)
$$

We introduce the unit normal vector $\mathbf{n}_{i j}$ to the edge $\left[P_{i}, P_{j}\right]: \mathbf{n}_{i j}=\frac{1}{\sqrt{a_{i, j}^{2}+b_{i, j}^{2}}}\left(\begin{array}{c}-b_{i, j} \\ a_{i, j}\end{array}\right)$. We consider a certain quantity $\mathbf{w}_{i}=\left(w_{i}^{x}, w_{i}^{y}\right) \in \mathbb{R}^{2}$ stored at the vertices. We associate to such a quantity the vector

$$
\mathbf{W}=\left(\begin{array}{c}
\mathbf{w}_{1} \\
\mathbf{w}_{2} \\
\cdots \\
\mathbf{w}_{n}
\end{array}\right) \in \mathbb{R}^{2} \times \mathbb{R}^{n} \simeq \mathbb{R}^{2 n}
$$


Let $U$ be the square matrix

$$
U=\left(\begin{array}{cc}
\frac{1}{2} & 0 \\
0 & \frac{1}{2}
\end{array}\right)
$$

We denote $A \in \mathfrak{M}_{2 n, 2 n}$ the non invertible matrix defined blockwise by

$$
A=\left(\begin{array}{cccccc}
U & U & 0 & 0 & \ldots & 0 \\
0 & U & U & 0 & \ldots & 0 \\
\vdots & & \ddots & \ddots & \ddots & \vdots \\
0 & \ldots & 0 & U & U & 0 \\
0 & \ldots & 0 & 0 & U & U \\
U & 0 & 0 & \ldots & 0 & U
\end{array}\right)
$$

For $P_{i}$ and $P_{j}$ two consecutive points of $\mathcal{P}$, we set $\mathbf{z}_{i, j}=\frac{\mathbf{w}_{i}+\mathbf{w}_{j}}{2} \in \mathbb{R}^{2}$ and we introduce the vector

$$
\mathbf{Z}=\left(\begin{array}{c}
\mathbf{z}_{1,2} \\
\mathbf{z}_{2,3} \\
\cdots \\
\mathbf{z}_{n-1, n} \\
\mathbf{z}_{n, 1}
\end{array}\right)=A \mathbf{W} \in \mathbb{R}^{2 n}
$$

Finally, let $\mathcal{N} \in \mathfrak{M}_{n, 2 n}$ be the matrix with $n$ rows and $2 n$ columns

$$
\mathcal{N}=\left(\begin{array}{ccccccccc}
\mathbf{n}_{1,2}^{x} & \mathbf{n}_{1,2}^{y} & 0 & 0 & \ldots & \ldots & & 0 & 0 \\
0 & 0 & \mathbf{n}_{2,3}^{x} & \mathbf{n}_{2,3}^{y} & 0 & 0 & \ldots & 0 & 0 \\
\vdots & \vdots & & & & & & \vdots & \vdots \\
0 & 0 & \ldots & \ldots & 0 & \mathbf{n}_{n-1, n}^{x} & \mathbf{n}_{n-1, n}^{y} & 0 & 0 \\
0 & 0 & \ldots & \ldots & 0 & 0 & 0 & \mathbf{n}_{n, 1}^{x} & \mathbf{n}_{n, 1}^{y}
\end{array}\right)
$$

We consider the linear application

$$
\begin{aligned}
\Phi: \mathbb{R}^{2 n} & \longrightarrow \mathbb{R}^{n} \\
\mathbf{W} & \longmapsto \mathcal{N} A \mathbf{W}
\end{aligned}
$$

The statement in Lemma 2.9 is equivalent to the claim: $\Phi$ is surjective. We introduce its transpose operator

$$
\begin{aligned}
\Phi^{\top}: \mathbb{R}^{n} & \longrightarrow \mathbb{R}^{2 n} \\
\mathbf{q} & \longmapsto A^{\top} \mathcal{N}^{\top} \mathbf{q}
\end{aligned}
$$

We are going to show that $\Phi^{\top}$ is injective. To this end let $\mathbf{q} \in \mathbb{R}^{n}$ such that $\Phi^{\top}(\mathbf{q})=\mathbf{0}$, that is to say

$$
\Phi^{\top}\left(\begin{array}{c}
q_{1} \\
q_{2} \\
\ldots \\
q_{n}
\end{array}\right)=\frac{1}{2}\left(\begin{array}{c}
q_{1} \mathbf{n}_{1,2}+q_{n} \mathbf{n}_{n, 1} \\
q_{1} \mathbf{n}_{1,2}+q_{2} \mathbf{n}_{2,3} \\
\ldots \\
\ldots \\
q_{n-1} \mathbf{n}_{n-1, n}+q_{n} \mathbf{n}_{n, 1}
\end{array}\right)=\mathbf{0} .
$$

As $K$ is a polygon with $n$ edges, there exist two consecutive edges $\left[P_{i}, P_{j}\right]$ and $\left[P_{j}, P_{k}\right]$ that are not on the same line; it follows that $\mathbf{n}_{i, j}$ and $\mathbf{n}_{j, k}$ are linearly independent. From the line $q_{i} \mathbf{n}_{i, j}+q_{j} \mathbf{n}_{j, k}=0$ of the above vector equality, we first deduce that $q_{i}=0$ and $q_{j}=0$ and then we successively obtain from the other lines that $\mathbf{q}=\mathbf{0}$, therefore $\operatorname{Ker}\left(\Phi^{\top}\right)=\{\mathbf{0}\}$ and $\Phi^{\top}$ is injective. Consequently, $\Phi$ is surjective and Lemma 2.9 is proved. 


\section{A few basic facts on $\mathbb{P}^{1}$ basis functions}

Let us remind the reader a few basic facts about the $\mathbb{P}^{1}$ basis functions used in Section 2.2.1. We consider a triangle $T$ whose vertices are denoted $P_{0}, P_{1}$ and $P_{2}$, see Fig. 13 at left. We are interested in the basis function $\phi_{0}$, which is $\mathbb{P}^{1}$ on the triangle $T$ and takes value 1 at $P_{0}$, while it vanishes on the other vertices $P_{1}$ and $P_{2}$. We thus have

$$
\phi_{0}(x, y)=a x+b y+c \text { for }(x, y) \in T,
$$

The constants $(a, b, c)$ are determined by solving the linear system

$$
\begin{aligned}
& a x_{0}+b y_{0}+c=1, \\
& a x_{1}+b y_{1}+c=0, \\
& a x_{2}+b y_{2}+c=0 .
\end{aligned}
$$

We are thus led to compute the following determinants

$$
D=\left|\begin{array}{lll}
x_{0} & y_{0} & 1 \\
x_{1} & y_{1} & 1 \\
x_{2} & y_{2} & 1
\end{array}\right|
$$

and

$$
D_{a}=\left|\begin{array}{lll}
1 & y_{0} & 1 \\
0 & y_{1} & 1 \\
0 & y_{2} & 1
\end{array}\right|, \quad D_{b}=\left|\begin{array}{lll}
x_{0} & 1 & 1 \\
x_{1} & 0 & 1 \\
x_{2} & 0 & 1
\end{array}\right|, \quad D_{c}=\left|\begin{array}{lll}
x_{0} & y_{0} & 1 \\
x_{1} & y_{1} & 0 \\
x_{2} & y_{2} & 0
\end{array}\right|
$$

We arrive at

$$
a=\frac{D_{a}}{D}, \quad b=\frac{D_{b}}{D}, \quad c=\frac{D_{c}}{D} .
$$

Note that $D$ is nothing but twice the area of the triangle $T$. Consequently, the gradient of $\phi_{0}$ is the constant vector given by

$$
\boldsymbol{\nabla} \phi_{0}=\left(\begin{array}{c}
D_{a} / D \\
D_{b} / D
\end{array}\right)=\frac{1}{2|T|}\left(\begin{array}{c}
y_{1}-y_{2} \\
x_{2}-x_{1}
\end{array}\right)=\frac{1}{2|T|}\left(\overrightarrow{P_{1} P_{2}}\right)^{\perp}
$$

which is therefore orthogonal to $P_{1} P_{2}$. This corresponds to the intuition: $(x, y) \mapsto$ $\phi_{0}(x, y)$ is non negative and it vanishes on the segment $\left[P_{1}, P_{2}\right]$ so that its gradient has no component along $\left(P_{1} P_{2}\right)$ and it points inward $T$. 

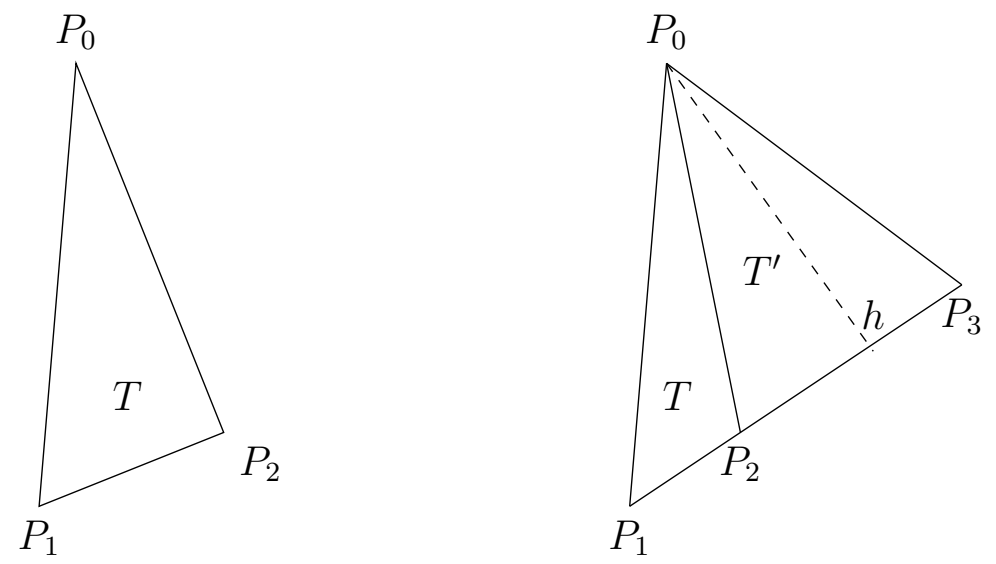

Figure 13: Properties of the piecewise $\mathbb{P}^{1}$ basis

We now consider two adjacents triangles $T=\left(P_{0} P_{1} P_{2}\right)$ and $T^{\prime}=\left(P_{0} P_{2} P_{3}\right)$ with $P_{1}, P_{2}$ and $P_{3}$ aligned as presented in Fig. 13 at right. We are again interested in the basis function $\phi_{0}$, which is piecewise $\mathbb{P}^{1}$ on the triangles $T, T^{\prime}$ and takes value 1 at $P_{0}$, while it vanishes on the other vertices $P_{1}, P_{2}$ and $P_{3}$. The computation above leads to

$$
\nabla \phi_{0 \mid T}=\frac{1}{2|T|}\left(\overrightarrow{P_{1} P_{2}}\right)^{\perp}, \quad \text { and } \quad \nabla \phi_{0 \mid T^{\prime}}=\frac{1}{2\left|T^{\prime}\right|}\left(\overrightarrow{P_{2} P_{3}}\right)^{\perp}
$$

In the specific case we are interested in, the three points $P_{1}, P_{2}, P_{3}$ are aligned, and thus $\boldsymbol{\nabla} \phi_{0 \mid T}$ and $\boldsymbol{\nabla} \phi_{0 \mid T^{\prime}}$ are colinear. Moreover, the two triangles $T$ and $T^{\prime}$ share the same height $h$ and we have $2|T|=h\left\|\overrightarrow{P_{1} P_{2}}\right\|$ and $2\left|T^{\prime}\right|=h\left\|\overrightarrow{P_{2} P_{3}}\right\|$. Let $\vec{u}$ be the unit vector, orthogonal to the axis $P_{1} P_{3}$ and pointing inward $T$ (and $T^{\prime}$ ). It follows that

$$
\nabla \phi_{0 \mid T}=\frac{\vec{u}}{h}=\nabla \phi_{0 \mid T^{\prime}}
$$

\section{References}

[1] B. Andreianov, M. Bendahmane, and K. Karlsen. A gradient reconstruction formula for

finite volume schemes and discrete duality. In R. Eymard and J.-M. Hérard, editors, Finite Volumes for Complex Applications V, Problems and Perspectives, pages 161-168. ISTE \& Wiley, 2008.

[2] B. Andreianov, F. Boyer, and F. Hubert. Discrete duality finite volume schemes for Leray-Lions-type elliptic problems on general 2D meshes. Numer. Methods Partial Differential Equations, 23(1):145-195, 2007.

[3] G. Ansanay-Alex, F. Babik, J.-C. Latché, and D. Vola. An $L^{2}$-stable approximation of the Navier-Stokes convection operator for low-order non-conforming finite elements. Int. J. Numer. Meth. Fluids, 66:555-580, 2011. 
[4] D. N. Arnold, L. R. Scott, and M. Vogelius. Regular inversion of the divergence operator with Dirichlet boundary conditions on a polygon. Annal. Scuola Normale Superiore Pisa - Classe di Scienze (Série 4), 15(2):169-192, 1988.

[5] F. Boyer, F. Dardalhon, C. Lapuerta, and J.-C. Latché. Stability of a CrankNicolson pressure correction scheme based on staggered discretizations. Int. J. Numer. Meth. Fluids, 74:34-58, 2014.

[6] C. Chainais-Hillairet and J. Droniou. Convergence analysis of a mixed finite volume scheme for an elliptic-parabolic system modeling miscible fluid flows in porous media. SIAM J. Numer. Anal., 45(5):2228-2258, 2007.

[7] Y. Coudière, J.-P. Vila, and P. Villedieu. Convergence rate of a finite volume scheme for a two-dimensional convection-diffusion problem. M2AN Math. Model. Numer. Anal., 33(3):493-516, 1999.

[8] S. Delcourte. Développement de méthodes de volumes finis pour la mécanique des fluides. PhD thesis, Université Paul Sabatier, Toulouse, France, 2007.

[9] S. Delcourte and P. Omnes. A discrete duality finite volume discretization of the vorticity-velocity-pressure formulation of the 2D Stokes problem on almost arbitrary two-dimensional grids. Numer. Meth. for PDEs, 31(1):1-30, 2015.

[10] K. Domelevo and P. Omnes. A finite volume method for the Laplace equation on almost arbitrary two-dimensional grids. M2AN Math. Model. Numer. Anal., 39(6):1203-1249, 2005.

[11] J. Droniou. Finite volume schemes for diffusion equations: Introduction to and review of modern methods. Math. Models Methods in Appl. Sc., 24(8):1575-1619, 2014.

[12] J. Droniou and R. Eymard. A mixed finite volume scheme for anisotropic diffusion problems on any grid. Numer. Math., 105:35-71, 2006.

[13] A. Ern and M. Vohralík. Stable broken $H^{1}$ and $H($ div $)$ polynomial extensions for polynomial-degree-robust potential and flux reconstruction in three space dimensions. Math. Comput., 89:551-594, 2020.

[14] A. Ern and M. Vohralík. Polynomial-degree-robust a posteriori estimates in a unified setting for conforming, nonconforming, discontinuous Galerkin, and mixed discretizations. SIAM J. Numer. Anal., 53(2):1058-1081, 2015.

[15] R. Eymard, T. Gallouët, and R. Herbin. Finite volume methods. In P. Ciarlet and J. Lions, editors, Handbook of numerical analysis, Vol. VII, Handb. Numer. Anal., VII, pages 715-1022. North-Holland, Amsterdam, 2000.

[16] R. Eymard, T. Gallouët, and R. Herbin. Finite volume approximation of elliptic problems and convergence of an approximate gradient. Appl. Numer. Math., $37(1-2): 31-53,2001$.

[17] T. Goudon and S. Krell. A DDFV scheme for incompressible Navier- Stokes equations with variable density. In J. Fuhrmann, M. Ohlberger, and C. Rohde, editors, Finite Volumes for Complex Applications VII-Elliptic, Parabolic and Hyperbolic Problems, pages 627-635. Springer International Publishing, 2014. 
[18] T. Goudon, J. Llobell, and S. Minjeaud. An explicit MUSCL-scheme on staggered grids with kinetic-like fluxes for the barotropic and full Euler systems. Comm. Comput. Phys., 27:672-724, 2020.

[19] T. Goudon, J. Llobell, and S. Minjeaud. A finite volume scheme on staggered grids for the Euler equations: unstructured meshes, stability analysis and energy conservation. Technical report, Univ. Côte d'Azur, Inria, CNRS, LJAD, 2020.

[20] P. Grisvard. Singularities in boundary value problems, volume 22 of Research Notes in Applied Math. Masson, 1992.

[21] F. Hermeline. A finite volume method for the approximation of diffusion operators on distorted meshes. J. Comput. Phys., 160(2):481-499, 2000.

[22] F. Hermeline. Approximation of diffusion operators with discontinuous tensor coefficients on distorted meshes. Comput. Methods Appl. Mech. Engrg., 192(1618):1939-1959, 2003.

[23] J. Llobell. Schémas Volumes Finis à mailles décalées pour la dynamique des gaz. PhD thesis, Université Côte d'Azur, France, 2018.

[24] J. C. Nagtegaal, D. M. Parks, and J. R. Rice. On numerically accurate finite element solution in the fully plastic range. Comput. Meth. Appl. Mech Engng., 4:153-177, 1974.

[25] P.-A. Raviart and J. M. Thomas. A mixed finite element method for 2nd order elliptic problems. In Mathematical aspects of finite element methods (Proc. Conf., Consiglio Naz. delle Ricerche (C.N.R.), Rome, 1975), pages 292-315. Lecture Notes in Math., Vol. 606, 1977.

[26] M. Vohralík. Residual flux-based a posteriori error estimates for finite volume and related locally conservative methods. Numer. Math., 111:121-158, 2008.

[27] M. Vohralík. Guaranteed and fully robust a posteriori error estimates for conforming discretizations of diffusion problems with discontinuous coefficients. J. Sci. Comput., 46:397-438, 2011. 